\title{
Chen's double sieve, Goldbach's conjecture and the twin prime problem
}

\author{
by
}

J. Wu (Nancy)

1. Introduction. Let $\Omega(n)$ be the number of all prime factors of the integer $n$ with the convention $\Omega(1)=0$. For an even integer $N \geq 4$, we define $D(N)$ as the number of representations of $N$ as a sum of two primes:

$$
D(N):=|\{p \leq N: \Omega(N-p)=1\}| ;
$$

here and in what follows, the letter $p$, with or without subscript, denotes a prime number. The well known Goldbach conjecture can be stated as $D(N) \geq 1$ for every even integer $N \geq 4$. A more precise version of this conjecture was proposed by Hardy \& Littlewood [15]:

$$
D(N) \sim 2 \Theta(N) \quad(N \rightarrow \infty),
$$

where

$$
\Theta(N):=\frac{C_{N} N}{(\log N)^{2}}, \quad C_{N}:=\prod_{p \mid N, p>2} \frac{p-1}{p-2} \prod_{p>2}\left(1-\frac{1}{(p-1)^{2}}\right) .
$$

Certainly, the asymptotic formula (1.1) is extremely difficult. Although the lower bound problem remains open, the upper bound problem has a rich history. In 1949 Selberg [25] proved

$$
D(N) \leq\{16+o(1)\} \Theta(N)
$$

with the help of his well known $\lambda^{2}$-upper bound sieve. By applying Linnik's large sieve method, C. D. Pan [20] in 1964 improved 16 to 12. In 1966, Bombieri \& Davenport [1] obtained 8 instead. Their proof is based on the linear sieve formulas and the mean value theorem of Bombieri-Vinogradov. It seems very difficult to prove (1.2) with a constant strictly less than 8 by the method of [1]. Firstly the linear sieve formulas (see Lemma 2.2 below)

$$
X V(z) f\left(\frac{\log Q}{\log z}\right)+\text { error } \leq S(\mathcal{A} ; \mathcal{P}, z) \leq X V(z) F\left(\frac{\log Q}{\log z}\right)+\text { error }
$$

2000 Mathematics Subject Classification: 11P32, 11N35, 11N05. 
are the best possible in the sense that for

$$
\mathcal{A}=\mathcal{B}_{\nu}:=\{n: 1 \leq n \leq x, \Omega(n) \equiv \nu(\bmod 2)\} \quad(\nu=1,2),
$$

the upper and lower bounds in (1.3) are respectively attained by $\nu=1$ and $\nu=2$ (see [14, p. 239]). Secondly it is hopeless to try to improve the level of distribution $\frac{1}{2}$ in Bombieri-Vinogradov's theorem.

In 1978, Chen [10] introduced a new idea in Selberg's sieve and proved

$$
D(N) \leq 7.8342 \Theta(N) \quad\left(N \geq N_{0}\right)
$$

His sieve machine involves two variables and is quite complicated. Roughly speaking, for the sequence

$$
\mathcal{A}=\{N-p: p \leq N\}
$$

he introduced two new functions $h(s)$ and $H(s)$ such that (1.3) holds with $f(s)+h(s)$ and $F(s)-H(s)$ in place of $f(s)$ and $F(s)$, respectively. The key innovation is to prove $h(s)>0$ and $H(s)>0$ via three weighted inequalities (see $[10,(23),(47),(64),(90)$, and (91)]). It is worth pointing out that he did not give complete proofs for these three inequalities. Among the three inequalities, the third one is the most complicated (with 43 terms) and it seems quite difficult to reconstruct a proof. Indeed, combining any one of these three inequalities with the Chen-Iwaniec switching principle (see [7] and [16]) leads to a constant less than 8. In order to derive a better result, Chen further introduced a very complicated iterative method. In 1980, C. B. Pan [19] applied essentially the first weighted inequality of Chen to get 7.988. According to [22], Chen's proof is very long and somewhat difficult to follow, but his idea is clear.

In this paper, inspired by the ideas in [26] we shall first try to give a more comprehensive treatment of Chen's double sieve and prove an upper bound sharper than (1.4).

TheOREM 1. For sufficiently large $N$, we have

$$
D(N) \leq 7.8209 \Theta(N) \text {. }
$$

The improvement comes from a new weighted inequality (see Lemma 4.2 below), which is still quite complicated with 21 terms, but much simpler than Chen's third and more powerful than his second and third inequality. Recently Cai \& Lu [6] gave another weighted inequality (with 31 terms), which is simpler but slightly weaker than Chen's third.

One way of approaching the lower bound problem in (1.1) is to give a nontrivial lower bound for the quantity

$$
D_{1,2}(N):=|\{p \leq N: \Omega(N-p) \leq 2\}| .
$$

In this direction, Chen [7] proved, by his system of weights and switching principle, the following famous theorem: Every sufficiently large even integer 
can be written as a sum of a prime and an integer having at most two prime factors. More precisely, he established

$$
D_{1,2}(N) \geq 0.67 \Theta(N) \quad\left(N \geq N_{0}\right) .
$$

Then Halberstam \& Richert [14] obtained a better constant 0.689 in place of 0.67 by a careful numerical calculation. As they indicated in [14], it would be interesting to know whether a more elaborate weighting procedure could be adapted to obtain (1.5). This might lead to numerical improvements and could be important. In 1978 Chen improved the constant 0.689 of Halberstam \& Richert to 0.7544 and to 0.81 by two more elaborate systems of weights ([8], [9]). Very recently by improving Chen's weighting device Cai and $\mathrm{Lu}[5]$ obtained 0.8285 , which they described as being near to the limit of what could be obtained by the method employed.

The second aim of this paper is to propose a larger constant.

TheOREM 2. For sufficiently large $N$, we have

$$
D_{1,2}(N) \geq 0.836 \Theta(N) .
$$

The proof of Theorem 2 is based on a modified version of Chen's weights (see Lemma 9.2 below), the linear sieve and the mean value theorems of Pan \& Ding [21] and of Fouvry [11].

A conjecture of the same nature is the twin prime problem, which can be stated as

$$
\pi_{2}(x):=|\{p \leq x: \Omega(p+2)=1\}| \rightarrow \infty \quad(x \rightarrow \infty) .
$$

Similarly to (1.1), Hardy \& Littlewood [15] conjectured

$$
\pi_{2}(x) \sim \Pi(x) \quad(x \rightarrow \infty),
$$

where

$$
\Pi(x):=\frac{C x}{(\log x)^{2}}, \quad C:=2 \prod_{p>2}\left(1-\frac{1}{(p-1)^{2}}\right) .
$$

The methods of Selberg, Pan, Bombieri \& Davenport and Chen work in a similar way and give upper bounds of the type

$$
\pi_{2}(x) \leq\{a+o(1)\} \Pi(x),
$$

where the constant $a$ is half the corresponding constant in the Goldbach problem. Due to the sieve of Rosser-Iwaniec and mean value theorems of Bombieri, Fouvry, Friedlander and Iwaniec, the history of (1.7) is much richer than that of (1.2). We refer the reader to [26] and [6] for a detailed historical description of this problem. In particular $\mathrm{Wu}[26]$ obtained 3.418 in place of $a+o(1)$ by placing these new mean value theorems in Chen's method. The main difficulty in applying these mean value theorems of [26] is to not destroy the fact that the error terms are affected by well factorisable 
coefficients. Recently Cai \& Lu [6] improved the constant 3.418 to 3.406 . Our argument in proving Theorem 1 allows us to give a better result.

Theorem 3. For sufficiently large $x$, we have

$$
\pi_{2}(x) \leq 3.3996 \Pi(x) .
$$

As an analogue of Theorem 2, Chen [7] proved that

$$
\pi_{1,2}(x) \geq 0.335 \Pi(x) \quad\left(x \geq x_{0}\right)
$$

where

$$
\pi_{1,2}(x):=|\{p \leq x: \Omega(p+2) \leq 2\}| .
$$

The constant 0.335 was improved by many mathematicians. Like (1.7), the history of (1.8) is much richer than that of (1.5). A detailed historical description of this problem can be found in the recent paper of Cai [3]. In particular he obtained 1.0974 in place of 0.335 , which is an improvement of Wu's constant 1.05 [26]. Here we can propose a slightly better result.

THEOREM 4. For sufficiently large $x$, we have

$$
\pi_{1,2}(x) \geq 1.104 \Pi(x) .
$$

Remark 1. (i) Theorems 1 and 3 show that the principal terms in the linear sieve formulas can be improved in the special cases $\mathcal{A}=\{N-p$ : $p \leq N\}$ or $\mathcal{A}=\{p+2: p \leq x\}$ (see the end of Section 3). This seems to be interesting and important. Our argument is quite general, and it works for all sequences satisfying the Chen-Iwaniec switching principle.

(ii) Certainly we could obtain a better constant than 3.3996 in Theorem 3 if we used mean value theorems of [11, Corollary 2], [12, Lemma 6] and [18, Proposition] as in the proof of Theorem 4. But the numerical computation involved would be quite complicated.

The Chen theorem in short intervals was first studied by Ross [23]. Let $\alpha \in(0,1)$ be a fixed constant and define, for $\theta \in(0,1), x \geq 2$ and even integer $N \geq 4$,

$$
\begin{aligned}
D_{1,2}(N, \theta) & :=\left|\left\{\alpha N \leq p \leq \alpha N+N^{\theta}: \Omega(N-p) \leq 2\right\}\right|, \\
\pi_{1,2}(x, \theta) & :=\left|\left\{x \leq p \leq x+x^{\theta}: \Omega(p+2) \leq 2\right\}\right| .
\end{aligned}
$$

He proved (see [23]) that for $\theta \geq 0.98, N \geq N_{0}(\theta)$ and $x \geq x_{0}(\theta)$,

$$
D_{1,2}(N, \theta) \gg \Xi(N, \theta), \quad \pi_{1,2}(x, \theta) \gg \Pi(x, \theta),
$$

where

$$
\Xi(N, \theta):=\frac{N^{\theta}}{(\log N)^{2}} \prod_{p \mid N, p>2} \frac{p-1}{p-2} \prod_{p>2}\left(1-\frac{1}{(p-1)^{2}}\right),
$$




$$
\Pi(x, \theta):=\frac{2 x^{\theta}}{(\log x)^{2}} \prod_{p>2}\left(1-\frac{1}{(p-1)^{2}}\right) .
$$

The constant 0.98 was further improved to 0.973 by $\mathrm{Wu}[28]$, to 0.9729 by Salerno \& Vitolo [24] and to 0.972 by Cai \& Lu [4].

Our method allows us to take a smaller exponent.

Theorem 5. For every $\theta \geq 0.971, N \geq N_{0}(\theta)$ and $x \geq x_{0}(\theta)$, we have

$$
D_{1,2}(N, \theta) \geq 0.012 \Xi(N, \theta), \quad \pi_{1,2}(x, \theta) \geq 0.006 \Pi(x, \theta) .
$$

Acknowledgements. The author would like to thank E. Fouvry for his generous help in writing this article, and the referee for his very careful reading of the manuscript.

2. Preliminary lemmas. This section is devoted to the formula of the Rosser-Iwaniec linear sieve and some mean value theorem on the distribution of primes in arithmetic progressions, which will be needed later. Before stating these results, it is necessary to recall some definitions.

Let $k$ be a positive integer and $\tau_{k}(n)$ the number of ways of writing $n$ as the product of $k$ positive integers. An arithmetical function $\lambda(q)$ is of level $Q$ and of order $k$ if

$$
\lambda(q)=0 \quad \text { for } q>Q \quad \text { and } \quad|\lambda(q)| \leq \tau_{k}(q) \quad \text { for } q \geq 1 .
$$

We say that $\lambda$ is well factorisable if for every decomposition $Q=Q_{1} Q_{2}$ $\left(Q_{1}, Q_{2} \geq 1\right)$ there exist two arithmetical functions $\lambda_{1}$ and $\lambda_{2}$ of level $Q_{1}, Q_{2}$ and of order $k$ such that $\lambda=\lambda_{1} * \lambda_{2}$.

LEMma 2.1. If $\lambda^{\prime}$ is an arithmetical function of level $Q^{\prime}(\leq Q)$ and of order $k^{\prime}$, then $\lambda * \lambda^{\prime}$ is well factorisable of level $Q Q^{\prime}$ and of order $k+k^{\prime}$.

Let $\mathcal{A}$ be a finite sequence of integers and $\mathcal{P}$ a set of prime numbers. For $z \geq 2$, we put $P(z):=\prod_{p<z, p \in \mathcal{P}} p$ and define the sieve function

$$
S(\mathcal{A} ; \mathcal{P}, z):=|\{a \in \mathcal{A}:(a, P(z))=1\}| .
$$

If $d$ is a square-free integer with all its prime factors belonging to $\mathcal{P}$, we denote by $\mathcal{A}_{d}$ the set of elements of $\mathcal{A}$ divisible by $d$ and we write the following approximate formula:

$$
\left|\mathcal{A}_{d}\right|=\frac{w(d)}{d} X+r(\mathcal{A}, d)
$$

where $X>1$ is independent of $d$, and $w(d)$ is a multiplicative function satisfying

$$
0 \leq w(p)<p \quad \text { for } p \in \mathcal{P} .
$$


We also define

$$
V(z):=\prod_{p<z}\left(1-\frac{w(p)}{p}\right)
$$

and suppose that there exists an absolute constant $K>1$ such that

$$
\frac{V\left(z_{1}\right)}{V\left(z_{2}\right)} \leq \frac{\log z_{2}}{\log z_{1}}\left(1+\frac{K}{\log z_{1}}\right) \quad\left(z_{2} \geq z_{1} \geq 2\right) .
$$

The formula of the Rosser-Iwaniec linear sieve [17] is stated as follows.

LEMMA 2.2. Let $0<\varepsilon<1 / 8$ and $2 \leq z \leq Q^{1 / 2}$. Under the assumptions (2.1)-(2.3), we have

$$
\begin{aligned}
& S(\mathcal{A} ; \mathcal{P}, z) \leq X V(z)\left\{F\left(\frac{\log Q}{\log z}\right)+E\right\}+\sum_{l<L} \sum_{q \mid P(z)} \lambda_{l}^{+}(q) r(\mathcal{A}, q), \\
& S(\mathcal{A} ; \mathcal{P}, z) \geq X V(z)\left\{f\left(\frac{\log Q}{\log z}\right)+E\right\}-\sum_{l<L} \sum_{q \mid P(z)} \lambda_{l}^{-}(q) r(\mathcal{A}, q) .
\end{aligned}
$$

In these formulas, $L$ depends only on $\varepsilon$, the $\lambda_{l}^{ \pm}$are well factorisable functions of order 1 and of level $Q$, and $E \ll \varepsilon+\varepsilon^{-8} e^{K} /(\log Q)^{1 / 3}$. The functions $F, f$ are defined by

$$
\begin{array}{cl}
F(u)=2 e^{\gamma} / u, & f(u)=0 \quad(0<u \leq 2), \\
(u F(u))^{\prime}=f(u-1), & (u f(u))^{\prime}=F(u-1) \quad(u>2),
\end{array}
$$

where $\gamma$ is Euler's constant.

As usual, we denote by $\mu(q)$ the Möbius function, $\varphi(q)$ Euler's function and $\nu(q)$ the number of distinct prime factors of $q$. Write

$$
\nu_{*}(q):=\mu(q)^{2} 3^{\nu(q)} .
$$

Define

$$
\bar{E}_{0}(y ; q, a, m):=\sum_{\substack{m p \leq y \\ m p \equiv a(\bmod q)}} 1-\frac{\operatorname{li}(y / m)}{\varphi(q)}
$$

where

$$
\operatorname{li}(y):=\int_{2}^{y} \frac{d t}{\log t}
$$

The next lemma is due to Pan \& Ding [21], which implies BombieriVinogradov's theorem. Here we state it in the form of [22, Corollary 8.12].

LEMMA 2.3. Let $f(m) \ll 1$ and $\alpha \in(0,1]$. Let $r_{1}(y)$ be a positive function depending on $x$ and satisfying

$$
r_{1}(y) \ll x^{\alpha}, \quad y \leq x .
$$


Let $r_{2}(m)$ be a positive function depending on $x$ and $y$, and satisfying

$$
m r_{2}(m) \ll x, \quad m \leq x^{\alpha}, \quad y \leq x .
$$

Then for every $A>0$, there exists a constant $B=B(A)>0$ such that

$$
\begin{aligned}
\sum_{q \leq \sqrt{x} /(\log x)^{B}} \nu_{*}(q) \max _{\substack{y \leq x \\
(a, q)=1}}\left|\sum_{\substack{m \leq x^{1-\alpha} \\
(m, q)=1}} f(m) \bar{E}_{0}(y ; q, a, m)\right| & \ll \frac{x}{(\log x)^{A}}, \\
\sum_{q \leq \sqrt{x} /(\log x)^{B}} \nu_{*}(q) \max _{\substack{y \leq x \\
(a, q)=1}}\left|\sum_{\substack{m \leq x^{1-\alpha} \\
(m, q)=1}} f(m) \bar{E}_{0}\left(m r_{1}(y) ; q, a, m\right)\right| & \ll \frac{x}{(\log x)^{A}}, \\
\sum_{q \leq \sqrt{x} /(\log x)^{B}} \nu_{*}(q) \max _{\substack{y \leq x \\
(a, q)=1}}\left|\sum_{\substack{m \leq x^{1-\alpha} \\
(m, q)=1}} f(m) \bar{E}_{0}\left(m r_{2}(m) ; q, a, m\right)\right| & \ll \frac{x}{(\log x)^{A}} .
\end{aligned}
$$

In order to prove Theorem 5 , it is necessary to generalize the mean value theorem of Pan \& Ding in short intervals. Such a result was established by $\mathrm{Wu}([27$, Theorem 2]).

Lemma 2.4. Let $f(m) \ll 1, \varepsilon$ be an arbitrarily small positive number and define

$$
H(y, h, q, a, m):=\sum_{\substack{y<m p \leq y+h \\ m p \equiv a(\bmod q)}} 1-\frac{\operatorname{li}((y+h) / m)-\operatorname{li}(y / m)}{\varphi(q)} .
$$

Then for any $A>0$, there exists a constant $B=B(A)>0$ such that

$$
\sum_{q \leq Q} \nu_{*}(q) \max _{\substack{x / 2<y \leq x \\ h \leq x^{\theta},(a, q)=1}}\left|\sum_{m \leq M,(m, q)=1} f(m) H(y, h, q, a, m)\right| \ll \frac{x^{\theta}}{(\log x)^{A}}
$$

for $x \geq 10,3 / 5+\varepsilon \leq \theta \leq 1, Q=x^{\theta-1 / 2} /(\log x)^{B}$ and $M=x^{(5 \theta-3) / 2-\varepsilon}$.

In the proofs of Theorems 3 and 4 , we shall need some mean value theorems with well factorisable or almost well factorisable coefficients.

Let $M \geq 1, N \geq 1$ and $X:=M N$. Let $\left\{\alpha_{m}\right\}$ and $\left\{\beta_{n}\right\}$ be two sequences of order $k$ supported in $[M, 2 M]$ and $[N, 2 N]$ respectively. We also suppose the conditions below:

(i) For all $B$, the equality

$$
\sum_{n \equiv n_{0}(\bmod k),(n, d)=1} \beta_{n}=\frac{1}{\varphi(k)} \sum_{(n, d k)=1} \beta_{n}+O_{B, k}\left(N \tau_{k}(d) /(\log 2 N)^{B}\right)
$$

holds for $d \geq 1, k \geq 1$ and $\left(k, n_{0}\right)=1$.

(ii) If $n$ has a prime factor $p$ with $p<\exp \left\{(\log \log n)^{2}\right\}$, then $\beta_{n}=0$. 
The following result is an immediate consequence of Corollary 2 of [11], Lemma 6 of [12] and the Proposition of [18].

Lemma 2.5. Under the conditions (i) and (ii) above, for any $A$ and for any $\varepsilon>0$ we have

$$
\sum_{(q, a)=1} \lambda(q)\left(\sum_{m n \equiv a(\bmod q)} \alpha_{m} \beta_{n}-\frac{1}{\varphi(q)} \sum_{(m n, q)=1} \alpha_{m} \beta_{n}\right) \ll_{\varepsilon, A} \frac{X}{(\log X)^{A}}
$$

uniformly for $|a| \leq(\log X)^{A}$ and $\nu:=\log N / \log X(\varepsilon \leq \nu \leq 1-\varepsilon)$. Here $\lambda(q)$ is a well factorisable function of order 1 and of level $Q:=X^{\theta(\nu)-\varepsilon}$, where $\theta(\nu)$ is given by

$$
\theta(\nu)= \begin{cases}(6-5 \nu) / 10 & \text { for } \varepsilon \leq \nu \leq 1 / 15 \\ 1 / 2+\nu & \text { for } 1 / 15 \leq \nu \leq 1 / 10 \\ (5-2 \nu) / 8 & \text { for } 1 / 10 \leq \nu \leq 3 / 14 \\ (3+2 \nu) / 6 & \text { for } 3 / 14 \leq \nu \leq 1 / 4 \\ (2-\nu) / 3 & \text { for } 1 / 4 \leq \nu \leq 2 / 7 \\ (2+\nu) / 4 & \text { for } 2 / 7 \leq \nu \leq 2 / 5 \\ 1-\nu & \text { for } 2 / 5 \leq \nu \leq 1 / 2, \\ 1 / 2 & \text { for } 1 / 2 \leq \nu \leq 1-\varepsilon\end{cases}
$$

Proof. The value $(6-5 \nu) / 10$ in $[\varepsilon, 1 / 15]$ comes from Corollary 2(ii) of [11]. The intervals $[1 / 15,1 / 10]$ and $[1 / 10,3 / 14]$ follow from the Proposition of [18] by decomposing $\lambda=\lambda_{1} * \lambda_{2}$ with

$$
Q_{1}=Q=x^{1 / 2-\varepsilon}, \quad Q_{2}=R=N x^{-\varepsilon}
$$

and

$$
Q_{1}=Q=x^{5 / 8-\varepsilon} N^{-5 / 4}, \quad Q_{2}=R=N x^{-\varepsilon},
$$

respectively. The remaining case is Lemma 6 of [12].

The next lemma is Corollary 2(i) of [11]. This is the first result which is valid uniformly for $|a| \leq X$ and has the level of distribution $>1 / 2$.

Lemma 2.6. Under the conditions (i) and (ii) above, for any $A$ and for any $\varepsilon>0$ we have

$$
\sum_{(q, a)=1} \lambda(q)\left(\sum_{m n \equiv a(\bmod q)} \alpha_{m} \beta_{n}-\frac{1}{\varphi(q)} \sum_{(m n, q)=1} \alpha_{m} \beta_{n}\right) \ll_{\varepsilon, A} \frac{X}{(\log X)^{A}}
$$

uniformly for $|a| \leq X$ and $\varepsilon \leq \nu:=\log N / \log X \leq 1 / 10$. Here $\lambda(q)$ is a well factorisable function of order 1 and of level $Q:=X^{5(1-\nu) 9-\varepsilon}$.

As usual define

$$
\pi(y ; q, a):=\sum_{p \leq y, p \equiv a(\bmod q)} 1 .
$$


The following result is due to Bombieri, Friedlander \& Iwaniec ([2, Theorem 10]).

LEMma 2.7. Let $\lambda$ be a well factorisable function of order $k$ and of level $Q=x^{4 / 7-\varepsilon}$. For any $\varepsilon>0$ and any $A$, we have uniformly for $x \geq 3$ and $|a| \leq(\log x)^{A}$,

$$
\sum_{(q, a)=1} \lambda(q)\left(\pi(x ; q, a)-\frac{\operatorname{li}(x)}{\varphi(q)}\right) \ll_{\varepsilon, k, A} \frac{x}{(\log x)^{A}} .
$$

When we use the weighted inequality, some coefficients are merely "almost well factorisable". So we need the following results, due to Fouvry \& Grupp ([13, Theorem 2 and the Corollary]).

LemMA 2.8. Let $\lambda$ be a well factorisable function of level $Q_{1}$ and of order $k, \xi$ an arithmetical function satisfying the conditions $\left|\xi\left(q_{2}\right)\right| \leq \log x$ and $\xi\left(q_{2}\right)=0\left(q_{2}>Q_{2}\right)$ and let $\Lambda$ be the von Mangoldt function. Then for any integer $a$, any $\varepsilon>0$ and any $A>0$,

$$
\sum_{\left(q_{1} q_{2}, a\right)=1} \lambda\left(q_{1}\right) \xi\left(q_{2}\right)\left(\pi\left(x ; q_{1} q_{2}, a\right)-\frac{\operatorname{li}(x)}{\varphi\left(q_{1} q_{2}\right)}\right) \ll_{a, \varepsilon, k, A} \frac{x}{(\log x)^{A}},
$$

provided one of the following three conditions is true:

$$
\begin{array}{ll}
Q_{2} \leq Q_{1}, & Q_{1} Q_{2} \leq x^{4 / 7-\varepsilon}, \\
Q_{2} \geq Q_{1}, & Q_{1} Q_{2}^{6} \leq x^{2-\varepsilon}, \\
\xi(q)=\Lambda(q), & Q_{1} Q_{2} \leq x^{11 / 20-\varepsilon}, \quad Q_{2} \leq x^{1 / 3-\varepsilon} .
\end{array}
$$

The next two lemmas also are useful when we apply the switching principle.

Lemma 2.9 ([26, Lemma 7]). Let $\lambda$ be a well factorisable function of level $Q:=x^{4 / 7-\varepsilon}$ and of order $k$. Let $\eta>0$ and $\left\{\varepsilon_{i}\right\}_{1 \leq i \leq r}$ be real numbers such that

$$
\varepsilon_{i} \geq \eta, \quad \varepsilon_{1}+\varepsilon_{2}+\cdots+\varepsilon_{r}=1 .
$$

Then for any integer $a$, any $\varepsilon>0$ and any $A>0$, we have

$$
\sum_{(q, a)=1} \lambda(q)\left(\sum_{\substack{p_{1} \cdots p_{r} \equiv a(\bmod q) \\ x^{\varepsilon_{i}}<p_{i} \leq 2 x^{\varepsilon_{i}}}} 1-\frac{1}{\varphi(q)} \sum_{\substack{\left(p_{1} \cdots p_{r}, q\right)=1 \\ x^{\varepsilon_{i}}<p_{i} \leq 2 x^{\varepsilon_{i}}}} 1\right) \ll_{a, \varepsilon, k, A} \frac{x}{(\log x)^{A}} .
$$

Lemma 2.10 ([26, Lemma 12]). Let $x \geq 2$ and $y=x^{1 / u}$. Then

$$
\sum_{\substack{n \leq x \\ p \mid n \Rightarrow p \geq y}} 1=\frac{x}{\log y} \omega(u)+O\left(\frac{x}{(\log y)^{2}}\right),
$$

where $\omega(u)$ is Buchstab's function defined by

$$
\omega(u)=1 / u \quad(1 \leq u \leq 2) \quad \text { and } \quad(u \omega(u))^{\prime}=\omega(u-1) \quad(u \geq 2) .
$$

Moreover, $\omega(u) \leq 0.561522(u \geq 3.5)$ and $\omega(u) \leq 0.567144(u \geq 2)$. 
3. Chen's double sieve. We shall sieve the sequence

$$
\mathcal{A}:=\{N-p: p \leq N\} .
$$

Let $\delta>0$ be a sufficiently small number and $k \in \mathbb{Z}$. Put

$$
Q:=N^{1 / 2-\delta}, \quad \underline{d}:=Q / d, \quad \mathcal{L}:=\log N, \quad W_{k}:=N^{\delta^{1+k}} .
$$

Let $\Delta$ be a real number with $1+\mathcal{L}^{-4} \leq \Delta<1+2 \mathcal{L}^{-4}$. We put $\mathcal{P}(N):=$ $\{p:(p, N)=1\}$ and denote by $\pi_{[Y, Z)}$ the characteristic function of the set $\mathcal{P}(N) \cap[Y, Z)$. For $k \in \mathbb{Z}^{+}$and $N \geq 2$, let $\mathfrak{U}_{k}(N)$ be the set of all arithmetical functions $\sigma$ which can be written in the form

$$
\sigma=\pi_{\left[V_{1} / \Delta, V_{1}\right)} * \cdots * \pi_{\left[V_{i} / \Delta, V_{i}\right)},
$$

where $i$ is an integer with $0 \leq i \leq k$, and $V_{1}, \ldots, V_{i}$ are real numbers satisfying

$$
\left\{\begin{array}{l}
V_{1}^{2} \leq Q, \\
V_{1} V_{2}^{2} \leq Q, \\
\cdots \cdots \cdots \cdots \\
V_{1} \cdots V_{i-1} V_{i}^{2} \leq Q, \\
V_{1} \geq \cdots \geq V_{i} \geq W_{k} .
\end{array}\right.
$$

By convention, $\sigma$ is the characteristic function of the set $\{1\}$ if $i=0$. From this definition and Lemma 2.1, we deduce immediately the following result.

Lemma 3.1. (i) We have $\mathfrak{U}_{k}(N) \subset \mathfrak{U}_{k+1}(N)$ for $k \in \mathbb{Z}^{+}$.

(ii) Let $\sigma=\pi_{\left[V_{1} / \Delta, V_{1}\right)} * \cdots * \pi_{\left[V_{i} / \Delta, V_{i}\right)} \in \mathfrak{U}_{k}(N)$. Then $\sigma$ is well factorisable of level $V:=V_{1} \cdots V_{i}$ and of order $i$. If $\lambda$ is well factorisable of level $Q / V$ and of order 1 , then $\sigma * \lambda$ is well factorisable of level $Q$ and of order $k+1$.

Let $F$ and $f$ be defined as in (2.6) and let

$$
A(s):=s F(s) / 2 e^{\gamma}, \quad a(s):=s f(s) / 2 e^{\gamma} .
$$

We introduce the notation

$$
\begin{aligned}
\Phi(N, \sigma, s) & :=\sum_{d} \sigma(d) S\left(\mathcal{A}_{d} ; \mathcal{P}(d N), \underline{d}^{1 / s}\right), \\
\Theta(N, \sigma) & :=4 \operatorname{li}(N) \sum_{d} \frac{\sigma(d) C_{d N}}{\varphi(d) \log \underline{d}} .
\end{aligned}
$$

For $k \in \mathbb{Z}^{+}, N_{0} \geq 2$ and $s \in[1,10]$, we define $H_{k, N_{0}}(s)$ and $h_{k, N_{0}}(s)$ as the supremum of $h \geq-\infty$ such that for all $N \geq N_{0}$ and $\sigma \in \mathfrak{U}_{k}(N)$ one has the following inequalities:

$$
\Phi(N, \sigma, s) \leq\{A(s)-h\} \Theta(N, \sigma), \quad \Phi(N, \sigma, s) \geq\{a(s)+h\} \Theta(N, \sigma)
$$

respectively. 
From this definition, we deduce immediately the following result.

Lemma 3.2. For $k \in \mathbb{Z}^{+}, N \geq N_{0}, s \in[1,10]$ and $\sigma \in \mathfrak{U}_{k}(N)$, we have

$$
\begin{aligned}
& \Phi(N, \sigma, s) \leq\left\{A(s)-H_{k, N_{0}}(s)\right\} \Theta(N, \sigma), \\
& \Phi(N, \sigma, s) \geq\left\{a(s)+h_{k, N_{0}}(s)\right\} \Theta(N, \sigma) .
\end{aligned}
$$

Obviously $H_{k, N_{0}}(s), h_{k, N_{0}}(s)$ are decreasing in $N_{0}$, and they are also decreasing in $k$ by Lemma 3.1. Hence we can write

$$
\begin{aligned}
H_{k}(s) & :=\lim _{N_{0} \rightarrow \infty} H_{k, N_{0}}(s), & h_{k}(s) & :=\lim _{N_{0} \rightarrow \infty} h_{k, N_{0}}(s), \\
H(s) & :=\lim _{k \rightarrow \infty} H_{k}(s), & h(s) & :=\lim _{k \rightarrow \infty} h_{k}(s) .
\end{aligned}
$$

Lemma 3.3. For $N \geq 2$ and $\sigma=\pi_{\left[V_{1} / \Delta, V_{1}\right)} * \cdots * \pi_{\left[V_{i} / \Delta, V_{i}\right)} \in \mathfrak{U}_{k}(N)$, we have

$$
\begin{aligned}
& \mathcal{L}^{-5 k} \ll_{\delta, k} \sum_{d} \sigma(d) / d \ll_{\delta, k} 1, \\
& \sum_{d} \sigma(d) \ll_{\delta, k} V_{1} \cdots V_{i}, \\
& \Theta(N, \sigma) \gg_{\delta, k} N / \mathcal{L}^{5 k+2} .
\end{aligned}
$$

Proof. Let $\sigma=\pi_{\left[V_{1} / \Delta, V_{1}\right)} * \cdots * \pi_{\left[V_{i} / \Delta, V_{i}\right)} \in \mathfrak{U}_{k}(N)$. We have

$$
\sum_{d} \frac{\sigma(d)}{d}=\prod_{1 \leq j \leq i} \sum_{p_{j} \in \mathcal{P}(N) \cap\left[V_{j} / \Delta, V_{j}\right)} \frac{1}{p_{j}} .
$$

The prime number theorem of the form

$$
\sum_{p \leq x} 1=\operatorname{li}(x)+O\left(x e^{-2 \sqrt{\log x}}\right)
$$

implies

$$
\begin{aligned}
\sum_{p_{j} \in \mathcal{P}(N) \cap\left[V_{j} / \Delta, V_{j}\right)} \frac{1}{p_{j}} & =\sum_{V_{j} / \Delta \leq p_{j}<V_{j}} \frac{1}{p_{j}}-\sum_{V_{j} / \Delta \leq p_{j}<V_{j}, p_{j} \mid N} \frac{1}{p_{j}} \\
& =\log \left(\frac{\log V_{j}}{\log \left(V_{j} / \Delta\right)}\right)+O\left(e^{-\log ^{1 / 2}\left(V_{j} / \Delta\right)}+\frac{\mathcal{L}}{V_{j} \log \mathcal{L}}\right) .
\end{aligned}
$$

Therefore our choice of $\Delta$ and (3.1) give

$$
\sum_{p_{j} \in \mathcal{P}(N) \cap\left[V_{j} / \Delta, V_{j}\right)} 1 / p_{j} \asymp_{\delta, k} \mathcal{L}^{-5} .
$$

Now (3.5) follows from (3.8) and (3.9).

Since $\sigma(d) \neq 0$ implies $d \leq V_{1} \cdots V_{i}$, the second inequality in (3.5) implies (3.6). Noticing $\Theta(N, \sigma) \gg N \mathcal{L}^{-2} \sum_{d} \sigma(d) / d$, we obtain (3.7) by the first inequality in (3.5). 
Proposition 1. For $k \in \mathbb{Z}^{+}$and $s \in[1,10]$, we have

$$
H_{k}(s) \geq 0, \quad h_{k}(s) \geq 0 .
$$

Proof. We shall prove only the first inequality. The second one can be treated similarly. Let $\sigma=\pi_{\left[V_{1} / \Delta, V_{1}\right)} * \cdots * \pi_{\left[V_{i} / \Delta, V_{i}\right)} \in \mathfrak{U}_{k}(N)$. We use Lemma 2.2 with

$$
X=\frac{\operatorname{li}(N)}{\varphi(d)}, \quad w(p)= \begin{cases}p /(p-1) & \text { if } p \in \mathcal{P}(N), \\ 0 & \text { otherwise }\end{cases}
$$

to estimate $\sigma(d) S\left(\mathcal{A}_{d} ; \mathcal{P}(d N), \underline{d}^{1 / s}\right)$. By Mertens's formula and (3.1), we infer that for any $\varepsilon>0$,

$$
V\left(\underline{d}^{1 / s}\right)=\left\{1+O_{\delta, k}(\varepsilon)\right\} \frac{2 s C_{d N}}{e^{\gamma} \log \underline{d}} .
$$

By using Lemma 2.2 and (3.10), we deduce

$$
\begin{aligned}
\sigma(d) S\left(\mathcal{A}_{d} ; \mathcal{P}(d N), \underline{d}^{1 / s}\right) \leq & 4 \operatorname{li}(N) \frac{\sigma(d) C_{d N}}{\varphi(d) \log \underline{d}} \\
& \times\left\{A\left(\frac{\log (Q / V)}{\log \underline{d}^{1 / s}}\right)+O_{\delta, k}(\varepsilon)\right\} \\
& +\sum_{l<L} \sigma(d) \sum_{q \mid P\left(\underline{d}^{1 / s}\right)} \lambda_{l}^{+}(q) r\left(\mathcal{A}_{d}, q\right),
\end{aligned}
$$

where $\lambda_{l}^{+}(q)$ is well factorisable of level $Q / V$ with $V:=V_{1} \cdots V_{i}$ and of order 1 .

If $\sigma(d) \neq 0$, we have $d \in\left[V / \Delta^{i}, V\right]$, which implies

$$
0 \leq \log V-\log d \leq i \log \Delta \leq 2 k \mathcal{L}^{-4} .
$$

From this we deduce that

$$
A\left(\log (Q / V) / \log \underline{d}^{1 / s}\right)=A(s)+O_{\delta, k}(\varepsilon) .
$$

Inserting (3.11) and summing over $d$, we obtain

$$
\Phi(N, \sigma, s) \leq\left\{A(s)+O_{\delta, k}(\varepsilon)\right\} \Theta(N, \sigma)+R,
$$

where

$$
R:=\sum_{l<L} \sum_{d} \sigma(d) \sum_{q \mid P\left(\underline{d}^{1 / s}\right)} \lambda_{l}^{+}(q) r\left(\mathcal{A}_{d}, q\right) .
$$

Let $q \mid P\left(\underline{d}^{1 / s}\right)$. It is clear that $\mu(q)^{2}=1$ and $(N d, q)=1$. Thus we have

$$
r\left(\mathcal{A}_{d}, q\right)=\left|\mathcal{A}_{d q}\right|-\operatorname{li}(N) / \varphi(d q)=\pi(N ; d q, N)-\operatorname{li}(N) / \varphi(d q) .
$$

Hence we can see, by using Lemmas 3.1(ii) and 2.3, that

$$
R \ll_{\varepsilon} \sum_{q \leq Q} \tau_{k+1}(q)|\pi(N ; d q, N)-\operatorname{li}(N) / \varphi(d q)| \ll_{\delta, k, \varepsilon} N / \mathcal{L}^{5 k+3}
$$


From (3.7), (3.12) and (3.13), we deduce that

$$
\Phi(N, \sigma, s) \leq\left\{A(s)+O_{\delta, k}(\varepsilon)\right\} \Theta(N, \sigma),
$$

which implies, by the definition of $H_{k, N_{0}}(s)$, for any $\varepsilon>0$ and sufficiently large $N_{0}$,

$$
H_{k, N_{0}}(s) \geq-O_{\delta, k}(\varepsilon) .
$$

First making $N_{0} \rightarrow \infty$ and then $\varepsilon \rightarrow 0$, we obtain $H_{k}(s) \geq 0$.

Proposition 2. For $2 \leq s \leq s^{\prime} \leq 10$, we have

$$
h(s) \geq h\left(s^{\prime}\right)+\int_{s-1}^{s^{\prime}-1} \frac{H(t)}{t} d t, \quad H(s) \geq H\left(s^{\prime}\right)+\int_{s-1}^{s^{\prime}-1} \frac{h(t)}{t} d t .
$$

Proof. We shall only prove the first inequality as the second one can be established in the same way.

Let $k \geq 0$ and $\sigma=\pi_{\left[V_{1} / \Delta, V_{1}\right)} * \cdots * \pi_{\left[V_{i} / \Delta, V_{i}\right)} \in \mathfrak{U}_{k}(N)$. By Buchstab's identity, we write

$$
\Phi(N, \sigma, s)=\Phi\left(N, \sigma, s^{\prime}\right)-\sum_{d} \sigma(d) \sum_{\underline{d}^{1 / s^{\prime}} \leq p<\underline{d}^{1 / s}} S\left(\mathcal{A}_{d p} ; \mathcal{P}(d N), p\right) .
$$

Next we shall give an upper bound for the last double sums $S$. The idea is to prove that the characteristic function of $d p$ belongs to $\mathfrak{U}_{k+1}(N)$. Thus $S$ can be estimated by a function $H_{k+1, N_{0}}$. We put $V:=V_{1} \cdots V_{i}, \underline{V}:=Q / V$ and $\alpha_{j}:=\underline{V}^{1 / s^{\prime}} \Delta^{j}$. Let $r$ be the integer satisfying $\alpha_{r} \leq \underline{V}^{1 / s}<\alpha_{r+1}$. Noticing that $\sigma(d) \neq 0 \Rightarrow \underline{V}^{1 / s^{\prime}} \leq \underline{d}^{1 / s^{\prime}}$ and $\underline{V}^{1 / s} \leq \underline{d}^{1 / s}$, we deduce

$$
\begin{aligned}
S & \leq \sum_{d} \sigma(d) \sum_{\alpha_{0} \leq p<\alpha_{r}} S\left(\mathcal{A}_{d p} ; \mathcal{P}(d N), p\right)+R_{1} \\
& =\sum_{1 \leq j \leq r} \sum_{d, p} \sigma(d) \pi_{\left[\alpha_{j-1}, \alpha_{j}\right)}(p) S\left(\mathcal{A}_{d p} ; \mathcal{P}(d p N),(\underline{d p})^{1 / s^{*}}\right)+R_{1},
\end{aligned}
$$

where $s^{*}:=\log \underline{d} / \log p-1$ and

$$
R_{1}:=\sum_{d} \sigma(d) \sum_{\alpha_{r} \leq p<\underline{d}^{1 / s}} S\left(\mathcal{A}_{d p} ; \mathcal{P}(d N), p\right) .
$$

We will prove that $\sigma * \pi_{\left[\alpha_{j-1}, \alpha_{j}\right)} \in \mathfrak{U}_{k+1}(N)$. It suffices to verify that $V_{1}, V_{2}, \ldots, V_{i}, \alpha_{j}$ satisfy (3.1) for $j \leq r$. If $V_{i} \geq \alpha_{j}$, then $V_{1} V_{2} \cdots V_{i} \alpha_{j}^{2} \leq$ $V \underline{V}^{2 / s}=Q^{2 / s} V^{1-2 / s} \leq Q$ and $\alpha_{j} \geq \underline{V}^{1 / s^{\prime}} \geq V_{i}^{1 / s^{\prime}} \geq W_{k}^{1 / s^{\prime}} \geq W_{k+1}$. If $V_{1} \geq \cdots \geq V_{l} \geq \alpha_{j} \geq V_{l+1} \geq \cdots \geq V_{i}$, we have $V_{1} \cdots V_{l} \alpha_{j} V_{l+1} \cdots V_{n}^{2} \leq$ $V \alpha_{j}^{2} \leq V \underline{V}^{2 / s} \leq Q$ for $l<n \leq i$. Thus $\sigma * \pi_{\left[\alpha_{j-1}, \alpha_{j}\right)} \in \mathfrak{U}_{k+1}(N)$.

Since $s^{*}$ depends on $d$ and $p$, we replace it by a suitable quantity independent of $d$ and $p$ such that we can use (3.3) with $H_{k+1, N_{0}}$. For this we introduce $s_{1}:=\log \left(\underline{V} / \alpha_{j}\right) / \log \alpha_{j}$ and $s_{2}:=\log \left(\underline{V} / \alpha_{j-i-1}\right) / \log \alpha_{j-1}$. 
Noticing that $\sigma(d) \pi_{\left[\alpha_{j-1}, \alpha_{j}\right)}(p) \neq 0 \Rightarrow s_{1} \leq s^{*} \leq s_{2}$, we deduce from (3.15) that

$$
S \leq \sum_{1 \leq j \leq r} \sum_{d, p} \sigma(d) \pi_{\left[\alpha_{j-1}, \alpha_{j}\right)}(p) S\left(\mathcal{A}_{d p} ; \mathcal{P}(d p N),\left(\underline{d p}^{1 / s_{1}}\right)+R_{1}+R_{2},\right.
$$

where

$$
\begin{aligned}
R_{2}:= & \sum_{1 \leq j \leq r} \sum_{d, p} \sigma(d) \pi_{\left[\alpha_{j-1}, \alpha_{j}\right)}(p)\left\{S\left(\mathcal{A}_{d p} ; \mathcal{P}(d p N),(\underline{d p})^{1 / s^{*}}\right)\right. \\
& \left.-S\left(\mathcal{A}_{d p} ; \mathcal{P}(d p N),(\underline{d p})^{1 / s_{1}}\right)\right\} .
\end{aligned}
$$

Now we can use (3.3) in Lemma 3.2 to write

$$
\begin{aligned}
S \leq & \sum_{1 \leq j \leq r}\left\{A\left(s_{1}\right)-H_{k+1, N_{0}}\left(s_{1}\right)\right\} \Theta\left(N, \sigma * \pi_{\left[\alpha_{j-1}, \alpha_{j}\right)}\right)+R_{1}+R_{2} \\
\leq & 4 \operatorname{li}(N) \sum_{d} \frac{\sigma(d) C_{d N}}{\varphi(d) \log \underline{d}} \sum_{\alpha_{0} \leq p<\alpha_{r}} \frac{A\left(s^{*}\right)-H_{k+1, N_{0}}\left(s^{*}\right)}{(p-2)(1-\log p / \log \underline{d})}+R_{1}+R_{2} \\
\leq & 4 \operatorname{li}(N) \sum_{d} \frac{\sigma(d) C_{d N}}{\varphi(d) \log \underline{d}} \sum_{\underline{d}^{1 / s^{\prime}} \leq p<\underline{d}^{1 / s}} \frac{A\left(s^{*}\right)-H_{k+1, N_{0}}\left(s^{*}\right)}{(p-2)(1-\log p / \log \underline{d})} \\
& +R_{1}+R_{2}+R_{3},
\end{aligned}
$$

where we have used the fact that $A(s)-H_{k+1, N_{0}}(s)$ is increasing in $s$, and the notation

$$
R_{3}:=4 \operatorname{li}(N) \sum_{d} \frac{\sigma(d) C_{d N}}{\varphi(d) \log \underline{d}} \sum_{\underline{V}^{1 / s^{\prime}} \leq p<\underline{d}^{1 / s^{\prime}}} \frac{A\left(s^{*}\right)-H_{k+1, N_{0}}\left(s^{*}\right)}{\varphi(p)(1-\log p / \log \underline{d})} .
$$

The prime number theorem and integration by parts show that

$$
\sum_{\underline{d}^{1 / s^{\prime}} \leq p<\underline{d}^{1 / s}} \frac{A\left(s^{*}\right)-H_{k+1, N_{0}}\left(s^{*}\right)}{(p-2)(1-\log p / \log \underline{d})}=\int_{s-1}^{s^{\prime}-1} \frac{A(t)-H_{k+1, N_{0}}(t)}{t} d t+O_{\delta, k}(\varepsilon) .
$$

Hence

$$
\begin{aligned}
S \leq & \left\{\int_{s-1}^{s^{\prime}-1} \frac{A(t)-H_{k+1, N_{0}}(t)}{t} d t+O_{\delta, k}(\varepsilon)\right\} \Theta(N, \sigma) \\
& +R_{1}+R_{2}+R_{3} .
\end{aligned}
$$

It remains to estimate $R_{1}, R_{2}, R_{3}$. Observing that $\sigma(d) \neq 0 \Rightarrow V / \Delta^{i} \leq$ $d<V$, we have $\underline{d}^{1 / s} \leq \underline{V}^{1 / s} \Delta^{i / s}$. Thus

$$
\log \left(\log \underline{d}^{1 / s} / \log \alpha_{r}\right) \leq \log \left(1+\log \Delta^{1+i / s} / \log \left(\underline{V}^{1 / s} / \Delta\right)\right) \ll_{\delta, k} \mathcal{L}^{-5} .
$$

By using the prime number theorem and the previous estimate, we have 


$$
\begin{aligned}
& R_{1} \ll \sum_{d} \sigma(d) \sum_{\alpha_{r} \leq p<\underline{d}^{1 / s}} N / d p \ll N \mathcal{L}^{-5} \sum_{d} \sigma(d) / \varphi(d) \\
& \ll \delta, k \Theta(N, \sigma) / \mathcal{L}^{3} .
\end{aligned}
$$

Similarly we can show that

$$
R_{3} \ll_{\delta, k} \Theta(N, \sigma) / \mathcal{L}^{3} .
$$

By the definition of $R_{2}$, we easily see that

$$
R_{2} \ll \sum_{d} \sigma(d) \sum_{\alpha_{0} \leq p<\alpha_{r}} \sum_{(\underline{d p})^{1 / s^{*}} \leq p^{\prime}<(\underline{d p})^{1 / s_{1}}} N / d p p^{\prime} .
$$

Using a similar argument, we can show that

$$
R_{2} \ll \delta, k \frac{N}{\mathcal{L}^{4}} \sum_{d} \frac{\sigma(d)}{\varphi(d) \log \underline{d}} \sum_{\underline{V}^{1 / s^{\prime}} \leq p<\underline{V}^{1 / s}} \frac{1}{p} \ll_{\delta, k} \frac{\Theta(N, \sigma)}{\mathcal{L}^{3}} .
$$

Combining (3.16)-(3.19), we obtain the desired upper bound, for $N \geq N_{0}$,

$$
S \leq\left\{\int_{s-1}^{s^{\prime}-1} \frac{A(t)-H_{k+1, N_{0}}(t)}{t} d t+O_{\delta, k}(\varepsilon)\right\} \Theta(N, \sigma) .
$$

Inserting it in (3.14), estimating the first sum on the right-hand side of (3.14) by (3.9) and noticing the relation

$$
a\left(s^{\prime}\right)-a(s)=\int_{s}^{s^{\prime}} A(t-1) d t
$$

we find that, for $N \geq N_{0}(\varepsilon, \delta, k)$,

$$
\Phi(N, \sigma, s) \geq\left\{a(s)+h_{k, N_{0}}\left(s^{\prime}\right)+\int_{s-1}^{s^{\prime}-1} \frac{H_{k+1, N_{0}}(t)}{t} d t+O_{\delta, k}(\varepsilon)\right\} \Theta(N, \sigma) .
$$

From the definition of $h_{k, N_{0}}(s)$, we deduce that, for any $\varepsilon>0$ and for sufficiently large $N_{0}$,

$$
h_{k, N_{0}}(s) \geq h_{k, N_{0}}\left(s^{\prime}\right)+\int_{s-1}^{s^{\prime}-1} \frac{H_{k+1, N_{0}}(t)}{t} d t+O_{\delta, k}(\varepsilon) .
$$

Taking $N_{0} \rightarrow \infty$ and then $\varepsilon \rightarrow 0$, we obtain

$$
h_{k}(s) \geq h_{k}\left(s^{\prime}\right)+\int_{s-1}^{s^{\prime}-1} \frac{H_{k+1}(t)}{t} d t
$$

which implies the required inequality. This completes the proof.

Corollary 1. The function $H(s)$ is decreasing on $[1,10]$. The function $h(s)$ is increasing on $[1,2]$ and is decreasing on $[2,10]$. 
Proof. According to the definition, we easily see that $H_{k, N_{0}}(s)$ is decreasing on $[1,3]$ since $A(s)=1$ for $1 \leq s \leq 3$. Thus $H(s)$ is also decreasing on $[1,3]$. When $3 \leq s \leq 10$, the required result follows immediately from Propositions 2 and 1.

Similarly the definition of $h_{k, N_{0}}(s)$ and the fact that $a(s)=0$ for $1 \leq$ $s \leq 2$ show that $h(s)$ is increasing on $[1,2]$. Propositions 2 and 1 imply that $h(s)$ is decreasing on $[2,10]$. This concludes the proof.

The central results in this section are Propositions 3 and 4 below. Before stating it, it is necessary to introduce some notation.

Let $1 \leq s \leq 3 \leq s^{\prime} \leq 5$ and $s \leq \kappa_{3} \leq \kappa_{2} \leq \kappa_{1} \leq s^{\prime}$. Define

$$
\begin{array}{ll}
\alpha_{1}:=\kappa_{1}-2, & \alpha_{2}:=s^{\prime}-2, \\
\alpha_{3}:=s^{\prime}-s^{\prime} / s-1, & \alpha_{4}:=s^{\prime}-s^{\prime} / \kappa_{2}-1, \\
\alpha_{5}:=s^{\prime}-s^{\prime} / \kappa_{3}-1, & \alpha_{6}:=s^{\prime}-2 s^{\prime} / \kappa_{2}, \\
\alpha_{7}:=s^{\prime}-s^{\prime} / \kappa_{1}-s^{\prime} / \kappa_{3}, & \alpha_{8}:=s^{\prime}-s^{\prime} / \kappa_{1}-s^{\prime} / \kappa_{2}, \\
\alpha_{9}:=\kappa_{1}-\kappa_{1} / \kappa_{2}-1 . &
\end{array}
$$

Let $\mathbf{1}_{[a, b]}(t)$ be the characteristic function of the interval $[a, b]$. We put

$$
\sigma(a, b, c):=\int_{a}^{b} \log \frac{c}{t-1} \frac{d t}{t}, \quad \sigma_{0}(t):=\frac{\sigma(3, t+2, t+1)}{1-\sigma(3,5,4)} .
$$

We can prove that $H(s)$ satisfies some functional inequalities.

Proposition 3. For $5 \geq s^{\prime} \geq 3 \geq s \geq 2$ and $s^{\prime}-s^{\prime} / s \geq 2$, we have

$$
H(s) \geq \Psi_{1}(s)+\int_{1}^{3} H(t) \Xi_{1}(t ; s) d t
$$

where $\Psi_{1}(s)$ is defined in Lemma 5.1 below and $\Xi_{1}(t ; s)=\Xi_{1}\left(t ; s, s^{\prime}\right)$ is given by

$$
\begin{aligned}
\Xi_{1}(t ; s):= & \frac{\sigma_{0}(t)}{2 t} \log \left(\frac{16}{(s-1)\left(s^{\prime}-1\right)}\right)+\frac{\mathbf{1}_{\left[\alpha_{2}, 3\right]}(t)}{2 t} \log \left(\frac{(t+1)^{2}}{(s-1)\left(s^{\prime}-1\right)}\right) \\
& +\frac{\mathbf{1}_{\left[\alpha_{3}, \alpha_{2}\right]}(t)}{2 t} \log \left(\frac{t+1}{(s-1)\left(s^{\prime}-1-t\right)}\right) .
\end{aligned}
$$

Proposition 4. Let $5 \geq s^{\prime} \geq 3 \geq s \geq 2$ and $s \leq \kappa_{3}<\kappa_{2}<\kappa_{1} \leq s^{\prime}$ satisfy

$$
s^{\prime}-s^{\prime} / s \geq 2, \quad 1 \leq \alpha_{i} \leq 3 \quad(1 \leq i \leq 9), \quad \alpha_{1}<\alpha_{4}, \quad \alpha_{5}<\alpha_{8} .
$$

Then

$$
H(s) \geq \Psi_{2}(s)+\int_{1}^{3} H(t) \Xi_{2}(t ; s) d t
$$


where $\Psi_{2}(s)$ is defined in Lemma 5.2 below, and $\Xi_{2}(t ; s)$ is given by

$$
\begin{aligned}
\Xi_{2}(t ; s):= & \frac{\sigma_{0}(t)}{5 t} \log \left(\frac{1024}{(s-1)\left(s^{\prime}-1\right)\left(\kappa_{1}-1\right)\left(\kappa_{2}-1\right)\left(\kappa_{3}-1\right)}\right) \\
& +\frac{\mathbf{1}_{\left[\alpha_{2}, 3\right]}(t)}{5 t} \log \left(\frac{(t+1)^{5}}{(s-1)\left(s^{\prime}-1\right)\left(\kappa_{1}-1\right)\left(\kappa_{2}-1\right)\left(\kappa_{3}-1\right)}\right) \\
& +\frac{\mathbf{1}_{\left[\alpha_{9}, \alpha_{1}\right]}(t)}{5 t} \log \left(\frac{t+1}{\left(\kappa_{2}-1\right)\left(\kappa_{1}-1-t\right)}\right) \\
& +\frac{\mathbf{1}_{\left[\alpha_{5}, \alpha_{2}\right]}(t)}{5 t} \log \left(\frac{t+1}{\left(\kappa_{3}-1\right)\left(s^{\prime}-1-t\right)}\right) \\
& +\frac{\mathbf{1}_{\left[\alpha_{3}, \alpha_{2}\right]}(t)}{5 t} \log \left(\frac{t+1}{(s-1)\left(s^{\prime}-1-t\right)}\right) \\
& +\frac{\mathbf{1}_{\left[\alpha_{1}, \alpha_{2}\right]}(t)}{5 t} \log \left(\frac{(t+1)^{2}}{\left(\kappa_{1}-1\right)\left(\kappa_{2}-1\right)}\right) \\
& +\frac{\mathbf{1}_{\left[\alpha_{7}, \alpha_{5}\right]}(t)}{5 t\left(1-t / s^{\prime}\right)} \log \left(\frac{s^{\prime 2}}{\left(\kappa_{1} s^{\prime}-s^{\prime}-\kappa_{1} t\right)\left(\kappa_{3} s^{\prime}-s^{\prime}-\kappa_{3} t\right)}\right) \\
& +\frac{\mathbf{1}_{\left[\alpha_{5}, \alpha_{8}\right]}(t)}{5 t\left(1-t / s^{\prime}\right)} \log \left(\frac{s^{\prime}\left(s^{\prime}-1-t\right)}{\kappa_{1} s^{\prime}-s^{\prime}-\kappa_{1} t}\right) \\
& +\frac{\mathbf{1}_{\left[\alpha_{6}, \alpha_{8}\right]}(t)}{5 t\left(1-t / s^{\prime}\right)} \log \left(\frac{s^{\prime}}{\kappa_{2} s^{\prime}-s^{\prime}-\kappa_{2} t}\right) \\
& +\frac{\mathbf{1}_{\left[\alpha_{8}, \alpha_{2}\right]}(t)}{5 t\left(1-t / s^{\prime}\right)} \log \left(s^{\prime}-1-t\right) .
\end{aligned}
$$

We shall prove these two propositions in Section 6. It is easy to see that $\Xi_{i}(t ; s)$ is positive and that for $s \in[1,3)$ there exist parameters $s^{\prime}, \kappa_{i}$ such that $\Psi_{i}(s)>0$. Therefore $H(s)>0$ for $s \in[1,3)$ and then Proposition 2 implies that $h(s)>0$ for $s \in[1,3)$. In Sections 7 and 8 , we shall give a numerical solution of (3.21) and (3.22), and prove Theorems 1 and 3.

4. Weighted inequalities for the sieve function. The aim of this section is to present two weighted inequalities for the sieve function. The first is essentially due to Chen $([10,(23)])$. The second is new, and it is not only much simpler than the third weighted inequality of Chen $([10,(64)$, (90) and (91)]) but also more powerful.

Lemma 4.1. Let $1 \leq s<s^{\prime} \leq 10$. For $N \geq 2, k \geq 0$ and $\sigma \in \mathfrak{U}_{k}(N)$, we have

$$
2 \Phi(N, \sigma, s) \leq \sum_{d} \sigma(d)\left(\Omega_{1}-\Omega_{2}+\Omega_{3}\right)+O_{\delta, k}\left(N^{1-\eta}\right),
$$

where $\eta=\eta(\delta, k)>0$ and $\Omega_{i}=\Omega_{i}(d)$ is given by 


$$
\begin{aligned}
& \Omega_{1}:=2 S\left(\mathcal{A}_{d} ; \mathcal{P}(d N), \underline{d}^{1 / s^{\prime}}\right), \\
& \Omega_{2}:=\sum_{\substack{\underline{d}^{1 / s^{\prime}} \leq p<\underline{d}^{1 / s} \\
(p, N)=1}} S\left(\mathcal{A}_{d p} ; \mathcal{P}(d N), \underline{d}^{1 / s^{\prime}}\right), \\
& \Omega_{3}:=\sum_{\substack{\underline{d}^{1 / s^{\prime}} \leq p_{1}<p_{2}<p_{3}<\underline{d}^{1 / s} \\
\left(p_{1} p_{2} p_{3}, N\right)=1}} S\left(\mathcal{A}_{d p_{1} p_{2} p_{3}} ; \mathcal{P}\left(d p_{1} N\right), p_{2}\right) .
\end{aligned}
$$

Proof. By the Buchstab identity, we have

$$
\begin{aligned}
& 2 S\left(\mathcal{A}_{d} ; \mathcal{P}(d N), \underline{d}^{1 / s}\right)=\Omega_{1}-2 \sum_{\substack{\underline{d}^{1 / s^{\prime} \leq p<d^{1 / s}} \\
(p, N)=1}} S\left(\mathcal{A}_{d p} ; \mathcal{P}(d N), p\right), \\
& \begin{array}{l}
\sum_{\substack{\underline{d}^{1 / s^{\prime}} \leq p_{1}<d^{1 / s} \\
\left(p_{1}, N\right)=1}} S\left(\mathcal{A}_{d p_{1}} ; \mathcal{P}(d N), p_{1}\right) \\
=\Omega_{0}+\sum_{\substack{\underline{d}^{1 / s^{\prime}} \leq p_{1} \leq p_{3}<\underline{d}^{1 / s} \\
\left(p_{1} p_{3}, N\right)=1}} S\left(\mathcal{A}_{d p_{1} p_{3}} ; \mathcal{P}\left(d p_{1} N\right), p_{3}\right),
\end{array} \\
& \begin{array}{l}
\sum_{\substack{\underline{d}^{1 / s^{\prime}} \leq p_{3}<\underline{d}^{1 / s} \\
\left(p_{3}, N\right)=1}} S\left(\mathcal{A}_{d p_{3}} ; \mathcal{P}(d N), p_{3}\right) \\
=\Omega_{2}-\sum_{\substack{\underline{d}^{1 / s^{\prime}} \leq p_{1}<p_{3}<\underline{d}^{1 / s} \\
\left(p_{1} p_{3}, N\right)=1}} S\left(\mathcal{A}_{d p_{1} p_{3}} ; \mathcal{P}(d N), p_{1}\right),
\end{array}
\end{aligned}
$$

where

$$
\Omega_{0}:=\sum_{\substack{\underline{d}^{1 / s^{\prime}} \leq p_{1}<\underline{d}^{1 / s} \\\left(p_{1}, N\right)=1}} S\left(\mathcal{A}_{d p_{1}} ; \mathcal{P}(d N), \underline{d}^{1 / s}\right) .
$$

Inserting (4.2) and (4.3) into (4.1), dropping the term $\Omega_{0}(\geq 0)$ and replacing $p_{1} \leq p_{3}$ by $p_{1}<p_{3}$, we find that

$$
2 S\left(\mathcal{A}_{d} ; \mathcal{P}(d N), \underline{d}^{1 / s}\right) \leq \Omega_{1}-\Omega_{2}+\Delta_{1},
$$

where

$$
\begin{aligned}
& \Delta_{1}:=\sum_{\substack{\underline{d}^{1 / s^{\prime}} \leq p_{1}<p_{3}<\underline{d}^{1 / s} \\
\left(p_{1} p_{3}, N\right)=1}}\left\{S\left(\mathcal{A}_{d p_{1} p_{3}} ; \mathcal{P}(d N), p_{1}\right)\right. \\
& \left.-S\left(\mathcal{A}_{d p_{1} p_{3}} ; \mathcal{P}\left(d p_{1} N\right), p_{3}\right)\right\} \\
& =\sum_{\substack{d^{1 / s^{\prime}} \leq p_{1} \leq p_{2}<p_{3}<\underline{d}^{1 / s} \\
\left(p_{1} p_{2} p_{3}, N\right)=1}} S\left(\mathcal{A}_{d p_{1} p_{2} p_{3}} ; \mathcal{P}\left(d p_{1} N\right), p_{2}\right) \text {. }
\end{aligned}
$$


By the inequality $S\left(\mathcal{A}_{d p_{1}^{2} p_{3}} ; \mathcal{P}(d N), p_{1}\right) \ll N / d p_{1}^{2} p_{3}$ and the fact that $\underline{d} \geq$ $W_{k}$, we easily see that

$$
\sum_{\underline{d}^{1 / s^{\prime}} \leq p_{1}<p_{3}<\underline{d}^{1 / s}} S\left(\mathcal{A}_{d p_{1}^{2} p_{3}} ; \mathcal{P}(d N), p_{1}\right) \ll \sum_{\substack{\underline{d}^{1 / s^{\prime}} \leq p_{1}<p_{3}<\underline{d}^{1 / s} \\ \ll \delta, k}} N / d p_{1}^{2} p_{3}
$$

for some $\eta=\eta(\delta, k)>0$. Inserting it in (4.4), we obtain

$$
\Delta_{1}=\Omega_{3}+O_{\delta, k}\left(N^{1-\eta} / d\right) .
$$

Finally, we complete the proof by applying (3.5).

Lemma 4.2. Let $1 \leq s \leq \kappa_{3}<\kappa_{2}<\kappa_{1} \leq s^{\prime} \leq 10$. For $N \geq 2, k \geq 0$ and $\sigma \in \mathfrak{U}_{k}(N)$, we have

$$
5 \Phi(N, \sigma, s) \leq \sum_{d} \sigma(d)\left(\Gamma_{1}-\cdots-\Gamma_{4}+\Gamma_{5}+\cdots+\Gamma_{21}\right)+O_{\delta, k}\left(N^{1-\eta}\right),
$$

where $\eta=\eta(\delta, k)>0$ and $\Gamma_{i}=\Gamma_{i}(d)$ is given by

$$
\begin{aligned}
& \Gamma_{1}:=4 S\left(\mathcal{A}_{d} ; \mathcal{P}(d N), \underline{d}^{1 / s^{\prime}}\right)+S\left(\mathcal{A}_{d} ; \mathcal{P}(d N), \underline{d}^{1 / \kappa_{1}}\right), \\
& \Gamma_{2}:=\sum_{\substack{\underline{d}^{1 / s^{\prime}} \leq p<d^{1 / s} \\
(p, N)=1}} S\left(\mathcal{A}_{d p} ; \mathcal{P}(d N), \underline{d}^{1 / s^{\prime}}\right), \\
& \Gamma_{3}:=\sum_{\substack{\underline{d}^{1 / s^{\prime}} \leq p<\underline{d}^{1 / \kappa_{2}} \\
(p, N)=1}} S\left(\mathcal{A}_{d p} ; \mathcal{P}(d N), \underline{d}^{1 / s^{\prime}}\right), \\
& \Gamma_{4}:=\sum_{\substack{\underline{d}^{1 / s^{\prime}} \leq p<\underline{d}^{1 / \kappa_{3}} \\
(p, N)=1}} S\left(\mathcal{A}_{d p} ; \mathcal{P}(d N), \underline{d}^{1 / s^{\prime}}\right), \\
& \Gamma_{5}:=\sum_{\substack{d^{1 / s^{\prime}} \leq p_{1}<p_{2}<d^{1 / \kappa_{2}} \\
\left(p_{1} p_{2}, N\right)=1}} S\left(\mathcal{A}_{d p_{1} p_{2}} ; \mathcal{P}(d N), \underline{d}^{1 / s^{\prime}}\right), \\
& \Gamma_{6}:=\sum_{\underline{d}^{1 / s^{\prime}} \leq p_{1}<\frac{d^{1 / \kappa_{1}}}{\left(p_{1} p_{2}, N\right)=1}} \sum_{\frac{d^{1 / \kappa_{2}} \leq p_{2}<\underline{d}^{1 / \kappa_{3}}}{\left(p^{\prime}\right.}} S\left(\mathcal{A}_{d p_{1} p_{2}} ; \mathcal{P}(d N), \underline{d}^{1 / s^{\prime}}\right), \\
& \Gamma_{7}:=\sum_{\substack{\underline{d}^{1 / s^{\prime}} \\
\underline{c}_{\left(p_{1}<p_{2}<p_{2}, N\right)=1}}} S\left(\mathcal{A}_{d p_{1} p_{2}} ; \mathcal{P}(d N), p_{1}\right), \\
& \Gamma_{8}:=\sum_{\substack{\underline{d}^{1 / s^{\prime}} \leq p_{1}<\underline{d}^{1 / \kappa_{1}} \leq p_{2}<\underline{d}^{1 / \kappa_{2}} \\
\left(p_{1} p_{2}, N\right)=1}} S\left(\mathcal{A}_{d p_{1} p_{2}} ; \mathcal{P}(d N), p_{1}\right),
\end{aligned}
$$




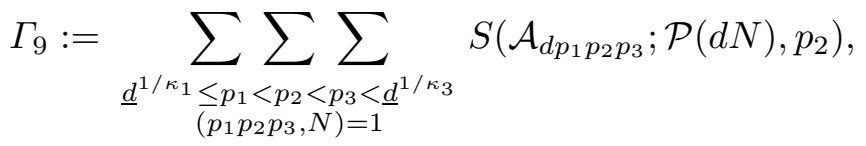

$$
\begin{aligned}
& \Gamma_{10}:=\sum_{\substack{d^{1 / \kappa_{1}} \leq p_{1}<p_{2}<\underline{d}^{1 / \kappa_{2}} \leq p_{3}<\underline{d}^{1 / s} \\
\left(p_{1} p_{2} p_{3}, N\right)=1}} S\left(\mathcal{A}_{d p_{1} p_{2} p_{3}} ; \mathcal{P}(d N), p_{2}\right), \\
& \left.\Gamma_{11}:=\sum_{\substack{d^{1 / \kappa_{1}} \leq p_{1}<\underline{d}^{1 / \kappa_{2}} \leq p_{2}<p_{3}<\underline{d}^{1 / \kappa_{3}} \\
\left(p_{1} p_{2} p_{3}, N\right)=1}} \sum_{\mathcal{A}_{d p_{1} p_{2} p_{3}}} ; \mathcal{P}(d N), p_{2}\right), \\
& \Gamma_{12}:=\sum_{\substack{\underline{d}^{1 / s^{\prime}} \leq p_{1}<p_{2}<\underline{d}^{1 / \kappa_{1}}, \underline{d}^{1 / \kappa_{3}} \leq p_{3}<\underline{d}^{1 / s} \\
\left(p_{1} p_{2} p_{3}, N\right)=1}} S\left(\mathcal{A}_{d p_{1} p_{2} p_{3}} ; \mathcal{P}(d N), p_{2}\right), \\
& \Gamma_{13}:=\sum_{\substack{\underline{d}^{1 / s^{\prime}} \leq p_{1}<\underline{d}^{1 / \kappa_{1}} \leq p_{2}<\underline{d}^{1 / \kappa_{2}} \leq p_{3}<\underline{d}^{1 / s} \\
\left(p_{1} p_{2} p_{3}, N\right)=1}} S\left(\mathcal{A}_{d p_{1} p_{2} p_{3}} ; \mathcal{P}(d N), p_{2}\right),
\end{aligned}
$$

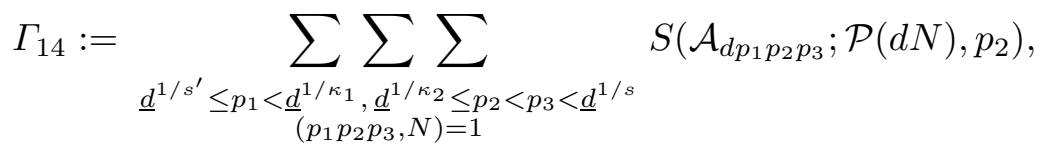

$$
\begin{aligned}
& \Gamma_{15}:=\sum_{\substack{\underline{d}^{1 / \kappa_{1}} \leq p_{1}<\underline{d}^{1 / \kappa_{2}} \leq p_{2}<\underline{d}^{1 / \kappa_{3}} \leq p_{3}<\underline{d}^{1 / s} \\
\left(p_{1} p_{2} p_{3}, N\right)=1}} S\left(\mathcal{A}_{d p_{1} p_{2} p_{3}} ; \mathcal{P}(d N), p_{2}\right), \\
& \left.\Gamma_{16}:=\sum_{\substack{d^{1 / \kappa_{2}} \leq p_{1}<p_{2}<p_{3}<p_{4}<\underline{d}^{1 / \kappa_{3}} \\
\left(p_{1} p_{2} p_{3} p_{4}, N\right)=1}} \sum_{\underline{A}{ }^{\prime} p_{1} p_{2} p_{3} p_{4}} ; \mathcal{P}(d N), p_{3}\right),
\end{aligned}
$$

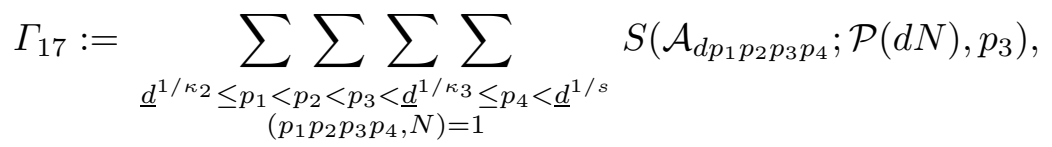

$$
\begin{aligned}
& \Gamma_{18}:=\sum_{\substack{d^{1 / \kappa_{2}} \leq p_{1}<p_{2}<\underline{d}^{1 / \kappa_{3}} \leq p_{3}<p_{4}<\underline{d}^{1 / s} \\
\left(p_{1} p_{2} p_{3} p_{4}, N\right)=1}} S\left(\mathcal{A}_{d p_{1} p_{2} p_{3} p_{4}} ; \mathcal{P}(d N), p_{3}\right),
\end{aligned}
$$

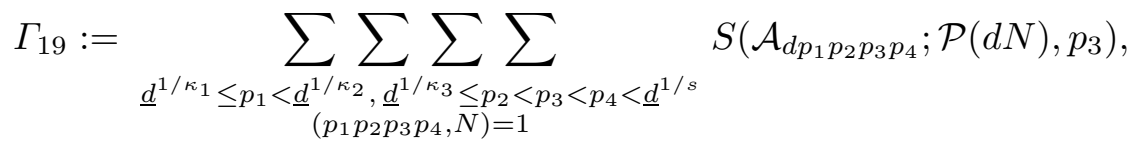

$$
\begin{aligned}
& \left.\Gamma_{20}:=\sum_{\substack{d^{1 / \kappa_{2}} \leq p_{1}<\underline{d}^{1 / \kappa_{3}} \leq p_{2}<p_{3}<p_{4}<p_{5}<\underline{d}^{1 / s} \\
\left(p_{1} p_{2} p_{3} p_{4} p_{5}, N\right)=1}} \sum_{\underline{A}^{\prime} p_{1} p_{2} p_{3} p_{4} p_{5}} ; \mathcal{P}(d N), p_{4}\right),
\end{aligned}
$$

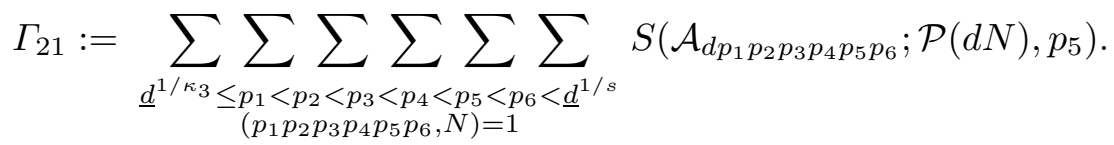


Proof. Let $S:=S\left(\mathcal{A}_{d} ; \mathcal{P}(d N), \underline{d}^{1 / s}\right)$. By the Buchstab identity, we have $(4.6)$

$$
\begin{aligned}
& 2 S=2 S\left(\mathcal{A}_{d} ; \mathcal{P}(d N), \underline{d}^{1 / s^{\prime}}\right)-\sum_{\substack{\underline{d}^{1 / s^{\prime}} \leq p<\underline{d}^{1 / s} \\
(p, N)=1}} S\left(\mathcal{A}_{d p} ; \mathcal{P}(d N), p\right) \\
& -\Gamma_{3}+\sum_{\substack{\underline{d}^{1 / s^{\prime}} \leq p_{1}<p_{2}<d^{1 / \kappa_{2}} \\
\left(p_{1} p_{2}, N\right)=1}} S\left(\mathcal{A}_{d p_{1} p_{2}} ; \mathcal{P}(d N), p_{1}\right) \\
& -\sum_{\substack{\underline{d}^{1 / \kappa_{2}} \leq p<\underline{d}^{1 / s} \\
(p, N)=1}} S\left(\mathcal{A}_{d p} ; \mathcal{P}(d N), p\right) \\
& =: 2 S\left(\mathcal{A}_{d} ; \mathcal{P}(d N), \underline{d}^{1 / s^{\prime}}\right)-E_{1}-\Gamma_{3}+D_{1}^{\prime}-E_{2} .
\end{aligned}
$$

We can also write, always by Buchstab's identity,

$$
\begin{aligned}
& S=S\left(\mathcal{A}_{d} ; \mathcal{P}(d N), \underline{d}^{1 / s^{\prime}}\right)-\sum_{\substack{\underline{d}^{1 / s^{\prime}} \leq p<\underline{d}^{1 / \kappa_{3}} \\
(p, N)=1}} S\left(\mathcal{A}_{d p} ; \mathcal{P}(d N), p\right) \\
& -\sum_{\substack{\underline{d}^{1 / \kappa_{3}} \leq p<\underline{d}^{1 / s} \\
(p, N)=1}} S\left(\mathcal{A}_{d p} ; \mathcal{P}(d N), p\right) .
\end{aligned}
$$

But we have

$$
\begin{aligned}
& \sum_{\substack{\underline{d}^{1 / s^{\prime}} \leq p<\underline{d}^{1 / \kappa_{3}} \\
(p, N)=1}} S\left(\mathcal{A}_{d p} ; \mathcal{P}(d N), p\right) \\
& =\Gamma_{4}-\Gamma_{7}-\Gamma_{8}-\sum_{\substack{\underline{d}^{1 / \kappa_{1}} \leq p_{1}<p_{2}<\underline{d}^{1 / \kappa_{3}} \\
\left(p_{1} p_{2}, N\right)=1}} S\left(\mathcal{A}_{d p_{1} p_{2}} ; \mathcal{P}(d N), p_{1}\right) \\
& -\Gamma_{6}+\sum_{\substack{\underline{d}^{1 / s^{\prime}} \leq p_{1}<p_{2}<\underline{d}^{1 / \kappa_{1}}, \underline{d}^{1 / \kappa_{2}} \leq p_{3}<\underline{d}^{1 / \kappa_{3}} \\
\left(p_{1} p_{2} p_{3}, N\right)=1}} S\left(\mathcal{A}_{d p_{1} p_{2} p_{3}} ; \mathcal{P}(d N), p_{1}\right) .
\end{aligned}
$$

Inserting these relations into (4.7) yields

$$
\begin{aligned}
S= & S\left(\mathcal{A}_{d} ; \mathcal{P}(d N), \underline{d}^{1 / s^{\prime}}\right)-\Gamma_{4}+\Gamma_{6}+\Gamma_{7}+\Gamma_{8} \\
& -\sum_{\substack{\underline{d}^{1 / \kappa_{3}} \leq p<\underline{d}^{1 / s} \\
(p, N)=1}} S\left(\mathcal{A}_{d p} ; \mathcal{P}(d N), p\right) \\
& +\sum_{\substack{\underline{d}^{1 / \kappa_{1}} \leq p_{1}<p_{2}<\underline{d}^{1 / \kappa_{3}} \\
\left(p_{1} p_{2}, N\right)=1}} S\left(\mathcal{A}_{d p_{1} p_{2}} ; \mathcal{P}(d N), p_{1}\right)
\end{aligned}
$$




$$
\begin{gathered}
-\sum_{\substack{\underline{d}^{1 / s^{\prime}} \leq p_{1}<p_{2}<\underline{d}^{1 / \kappa_{1}}, \underline{d}^{1 / \kappa_{2}} \leq p_{3}<\underline{d}^{1 / \kappa_{3}} \\
\left(p_{1} p_{3}, N\right)=1}} S\left(\mathcal{A}_{d p_{1} p_{2} p_{3}} ; \mathcal{P}(d N), p_{1}\right) \\
=: S\left(\mathcal{A}_{d} ; \mathcal{P}(d N), \underline{d}^{1 / s^{\prime}}\right)-\Gamma_{4}+\Gamma_{6}+\Gamma_{7}+\Gamma_{8}-E_{3}+D_{2}-E_{4} .
\end{gathered}
$$

Similarly to (4.8), we can prove that

$$
\begin{aligned}
S= & S\left(\mathcal{A}_{d} ; \mathcal{P}(d N), \underline{d}^{1 / s^{\prime}}\right)-\Gamma_{2}+\Gamma_{5} \\
& -\sum_{\underline{d}^{1 / s^{\prime}} \leq p_{1}<p_{2}<p_{3}<\underline{d}^{1 / \kappa_{2}}} S\left(\mathcal{A}_{d p_{1} p_{2} p_{3}} ; \mathcal{P}(d N), p_{1}\right) \\
& +\left\{\sum_{\substack{\left(p_{1} p_{2} p_{3}, N\right)=1 \\
\underline{d}^{1 / s^{\prime}} \leq p_{1}<\underline{d}^{1 / \kappa_{2}} \leq p_{2}<\underline{d}^{1 / s} \\
\left(p_{1} p_{2}, N\right)=1}}+\sum_{\substack{\underline{d}^{1 / \kappa_{2}} \leq p_{1}<p_{2}<\underline{d}^{1 / s} \\
\left(p_{1} p_{2}, N\right)=1}} \sum_{=:}\right. \\
& \times S\left(\mathcal{A}_{d} ; \mathcal{P}(d N), \underline{d}^{1 / s^{\prime}}\right)-\Gamma_{2}+\Gamma_{5}-E_{5}+D_{1}^{\prime \prime}+D_{1}^{\prime \prime \prime} .
\end{aligned}
$$

Finally, we write

$$
\begin{aligned}
S= & S\left(\mathcal{A}_{d} ; \mathcal{P}(d N), \underline{d}^{1 / \kappa_{1}}\right) \\
& -\left\{\sum_{\substack{\underline{d}^{1 / \kappa_{1}} \leq p<\underline{d}^{1 / \kappa_{3}} \\
(p, N)=1}}+\sum_{\substack{\underline{d}^{1 / \kappa_{3}} \leq p<\underline{d}^{1 / s} \\
(p, N)=1}}\right\} S\left(\mathcal{A}_{d p} ; \mathcal{P}(d N), p\right) .
\end{aligned}
$$

For $p_{1}<\underline{d}^{1 / \kappa_{3}}<\underline{d}^{1 / s}$, we have

$$
\begin{aligned}
S\left(\mathcal{A}_{d p} ; \mathcal{P}(d N), p\right) \geq & \sum_{\substack{p \leq p_{1}<d^{1 / \kappa_{3}} \\
\left(p_{1}, N\right)=1}} S\left(\mathcal{A}_{d p p_{1}} ; \mathcal{P}(d N), p_{1}\right) \\
& +\sum_{\substack{\underline{d}^{1 / \kappa_{3}} \leq p_{1}<\underline{d}^{1 / s} \\
\left(p_{1}, N\right)=1}} S\left(\mathcal{A}_{d p p_{1}} ; \mathcal{P}(d N), p_{1}\right) .
\end{aligned}
$$

This implies

$$
\begin{aligned}
& \sum_{\substack{\underline{d}^{1 / \kappa_{1}} \leq p<\underline{d}^{1 / \kappa_{3}} \\
(p, N)=1}} S\left(\mathcal{A}_{d p} ; \mathcal{P}(d N), p\right) \\
& \geq \sum_{\substack{\underline{d}^{1 / \kappa_{1}} \leq p_{1}<p_{2}<\underline{d}^{1 / \kappa_{3}} \\
\left(p_{1} p_{2}, N\right)=1}} S\left(\mathcal{A}_{d p_{1} p_{2}} ; \mathcal{P}(d N), p_{2}\right) \\
&+\sum_{\substack{\underline{d}^{1 / \kappa_{1}} \leq p_{1}<\underline{d}^{1 / \kappa_{3}} \leq p_{2}<\underline{d}^{1 / s} \\
\left(p_{1} p_{2}, N\right)=1}} S\left(\mathcal{A}_{d p_{1} p_{2}} ; \mathcal{P}(d N), p_{2}\right) .
\end{aligned}
$$


Inserting it into (4.10), we obtain

$$
\begin{aligned}
& S \leq S\left(\mathcal{A}_{d} ; \mathcal{P}(d N), \underline{d}^{1 / \kappa_{1}}\right) \\
&-\sum_{\substack{\underline{d}^{1 / \kappa_{1}} \leq p_{1}<p_{2}<\underline{d}^{1 / \kappa_{3}} \\
\left(p_{1} p_{2}, N\right)=1}} S\left(\mathcal{A}_{d p_{1} p_{2}} ; \mathcal{P}(d N), p_{2}\right) \\
&-\sum_{\substack{\underline{d}^{1 / \kappa_{1}} \leq p_{1}<\underline{d}^{1 / \kappa_{3}} \leq p_{2}<\underline{d}^{1 / s} \\
\left(p_{1} p_{2}, N\right)=1}} S\left(\mathcal{A}_{d p_{1} p_{2}} ; \mathcal{P}(d N), p_{2}\right) \\
&-\sum_{\substack{\underline{d}^{1 / \kappa_{3}} \leq p<\underline{d}^{1 / s} \\
(p, N)=1}} S\left(\mathcal{A}_{d p} ; \mathcal{P}(d N), p\right) \\
&=: S\left(\mathcal{A}_{d} ; \mathcal{P}(d N), \underline{d}^{1 / \kappa_{1}}\right)-E_{6}-E_{7}-E_{3} .
\end{aligned}
$$

Now by adding up the inequalities (4.6), (4.8), (4.9) and (4.11) and by noticing the estimate $D_{2}-E_{6} \leq \Gamma_{9}+O_{\delta, k}\left(N^{1-\eta} / d\right)$, we get

$$
5 S \leq \Gamma_{1}-\Gamma_{2}-\Gamma_{3}-\Gamma_{4}+\Gamma_{5}+\cdots+\Gamma_{9}+\Delta_{2}+O_{\delta, k}\left(N^{1-\eta} / d\right),
$$

where

$$
\Delta_{2}:=D_{1}-E_{1}-E_{2}-2 E_{3}-E_{4}-E_{5}-E_{7}
$$

and

$$
D_{1}:=D_{1}^{\prime}+D_{1}^{\prime \prime}+D_{1}^{\prime \prime \prime}=\sum_{\substack{\underline{d}^{1 / s^{\prime}} \leq p_{1}<p_{2}<\underline{d}^{1 / s} \\\left(p_{1} p_{2}, N\right)=1}} S\left(\mathcal{A}_{d p_{1} p_{2}} ; \mathcal{P}(d N), p_{1}\right) .
$$

Clearly we have

$$
E_{1} \geq \sum_{\substack{\underline{d}^{1 / s^{\prime}} \leq p_{1}<p_{2}<d^{1 / s} \\\left(p_{1} p_{2}, N\right)=1}} S\left(\mathcal{A}_{d p_{1} p_{2}} ; \mathcal{P}(d N), p_{2}\right) .
$$

Thus an application of Buchstab's identity gives

$$
D_{1}-E_{1} \leq \sum_{\substack{d^{1 / s^{\prime}} \leq p_{1}<p_{2}<p_{3}<\underline{d}^{1 / s} \\\left(p_{1} p_{2} p_{3}, N\right)=1}} S\left(\mathcal{A}_{d p_{1} p_{2} p_{3}} ; \mathcal{P}(d N), p_{2}\right)+O_{\delta, k}\left(N^{1-\eta} / d\right) .
$$

From this, we can deduce

$$
\begin{aligned}
D_{1}-E_{1}-E_{5} \leq & \sum_{\substack{\underline{d}^{1 / s^{\prime}} \leq p_{1}<p_{2}<p_{3}<\underline{d}^{1 / s} \\
\left(p_{1} p_{2} p_{3}, N\right)=1}} S\left(\mathcal{A}_{d p_{1} p_{2} p_{3}} ; \mathcal{P}(d N), p_{2}\right) \\
& -\sum_{\substack{\underline{d}^{1 / s^{\prime}} \leq p_{1}<p_{2}<p_{3}<\underline{d}^{1 / \kappa_{2}} \\
\left(p_{1} p_{2} p_{3}, N\right)=1}} S\left(\mathcal{A}_{d p_{1} p_{2} p_{3}} ; \mathcal{P}(d N), p_{2}\right) \\
& +O_{\delta, k}\left(N^{1-\eta} / d\right)
\end{aligned}
$$




$$
\begin{aligned}
= & \sum_{\substack{\underline{d}^{1 / s^{\prime}} \leq p_{1}<p_{2}<\underline{d}^{1 / \kappa_{2}} \leq p_{3}<\underline{d}^{1 / s} \\
\left(p_{1} p_{2} p_{3}, N\right)=1}} S\left(\mathcal{A}_{d p_{1} p_{2} p_{3}} ; \mathcal{P}(d N), p_{2}\right) \\
& +\sum_{\substack{\underline{d}^{1 / s^{\prime}} \leq p_{1}<\underline{d}^{1 / \kappa_{2}} \leq p_{2}<p_{3}<\underline{d}^{1 / s} \\
\left(p_{1} p_{2} p_{3}, N\right)=1}} S\left(\mathcal{A}_{d p_{1} p_{2} p_{3}} ; \mathcal{P}(d N), p_{2}\right) \\
& +\sum_{\substack{\underline{d}^{1 / \kappa_{2}} \leq p_{1}<p_{2}<p_{3}<\underline{d}^{1 / s} \\
\left(p_{1} p_{2} p_{3}, N\right)=1}} S\left(\mathcal{A}_{d p_{1} p_{2} p_{3}} ; \mathcal{P}(d N), p_{2}\right) \\
& +O_{\delta, k}\left(N^{1-\eta} / d\right) \\
= & : D_{3}+D_{4}+D_{5}+O_{\delta, k}\left(N^{1-\eta} / d\right) .
\end{aligned}
$$

We have

$$
D_{3}-E_{4} \leq \Gamma_{10}+\Gamma_{12}+\Gamma_{13}
$$

By splitting $D_{4}$ into 4 subsums, we have

$$
\begin{aligned}
& D_{4}=\Gamma_{11}+\Gamma_{14}+\Gamma_{15} \\
& +\sum_{\underline{d}^{1 / \kappa_{1}} \leq p_{1}<\frac{d^{1 / \kappa_{2}}, \underline{d}^{1 / \kappa_{3}} \leq p_{2}<p_{3}<\underline{d}^{1 / s}}{\left(p_{1} p_{2} \underline{p}_{3}, N\right)=1}} S\left(\mathcal{A}_{d p_{1} p_{2} p_{3}} ; \mathcal{P}(d N), p_{2}\right) .
\end{aligned}
$$

Similarly by splitting $E_{7}$ into 2 subsums, we have

$$
\begin{aligned}
& E_{7}=E_{8}+\sum_{\underline{d}^{1 / \kappa_{1}} \leq p_{1}<\underline{d}^{1 / \kappa_{2}}, \underline{d}^{1 / \kappa_{3}} \leq p_{2}<\underline{d}^{1 / s}} S\left(\mathcal{A}_{d p_{1} p_{2}} ; \mathcal{P}(d N), p_{2}\right) \\
& \geq E_{8}+\sum_{\underline{d}^{1 / \kappa_{1}} \leq p_{1}<\frac{d^{1 / \kappa_{2}}, \underline{d}^{1 / \kappa_{3}} \leq p_{2}<p_{3}<\underline{d}^{1 / s}}{\left(p_{1} p_{2} \underline{p}_{3}, N\right)=1}} S\left(\mathcal{A}_{d p_{1} p_{2} p_{3}} ; \mathcal{P}(d N), p_{3}\right),
\end{aligned}
$$

where

$$
E_{8}:=\sum_{\substack{\underline{d}^{1 / \kappa_{2}} \leq p_{1}<\underline{d}^{1 / \kappa_{3}} \leq p_{2}<\underline{d}^{1 / s} \\\left(p_{1} p_{2}, N\right)=1}} S\left(\mathcal{A}_{d p_{1} p_{2}} ; \mathcal{P}(d N), p_{2}\right) .
$$

By noticing that

$$
\begin{aligned}
\sum_{\underline{d}^{1 / \kappa_{1}} \leq p_{1}<\underline{d}^{1 / \kappa_{2}}, \underline{d}^{1 / \kappa_{3}} \leq p_{2}<p_{3}<\underline{d}^{1 / s}} & \left\{S\left(\mathcal{A}_{d p_{1} p_{2} p_{3}} ; \mathcal{P}(d N), p_{2}\right)\right. \\
\left.p_{1} p_{2} p_{3}, N\right)=1 & \left.-S\left(\mathcal{A}_{d p_{1} p_{2} p_{3}} ; \mathcal{P}(d N), p_{3}\right)\right\} \\
= & \Gamma_{19}+O_{\delta, k}\left(N^{1-\eta} / d\right),
\end{aligned}
$$

we can deduce

$$
D_{4}-E_{7} \leq \Gamma_{11}+\Gamma_{14}+\Gamma_{15}+\Gamma_{19}-E_{8}+O_{\delta, k}\left(N^{1-\eta} / d\right) .
$$


Since

$$
E_{2} \geq \sum_{\substack{\underline{d}^{1 / \kappa} \leq_{2} \leq p_{1}<p_{2}<p_{3}<\underline{d}^{1 / s} \\\left(p_{1} p_{2} p_{3}, N\right)=1}} S\left(\mathcal{A}_{d p_{1} p_{2} p_{3}} ; \mathcal{P}(d N), p_{3}\right)
$$

we have

$$
\begin{aligned}
& \left.D_{5}-E_{2} \leq \sum_{\substack{d^{1 / \kappa_{2}} \leq p_{1}<p_{2}<p_{3}<p_{4}<\underline{d}^{1 / s} \\
\left(p_{1} p_{2} p_{3} p_{4}, N\right)=1}} \sum_{\underline{A}_{d p_{1} p_{2} p_{3} p_{4}}} ; \mathcal{P}(d N), p_{3}\right) \\
& +O_{\delta, k}\left(N^{1-\eta} / d\right) \\
& =: D_{6}+O_{\delta, k}\left(N^{1-\eta} / d\right) \text {. }
\end{aligned}
$$

Similarly

$$
\begin{aligned}
& \left.E_{3} \geq \sum_{\substack{d^{1 / \kappa_{3}} \leq p_{1} \leq p_{2}<p_{3}<p_{4}<\underline{d}^{1 / s} \\
\left(p_{1} p_{2} p_{3} p_{4}, N\right)=1}} \sum_{\mathcal{A}} \sum_{\mathcal{A}_{1} p_{2} p_{3} p_{4}} ; \mathcal{P}(d N), p_{4}\right)=: E_{3}^{\prime},
\end{aligned}
$$

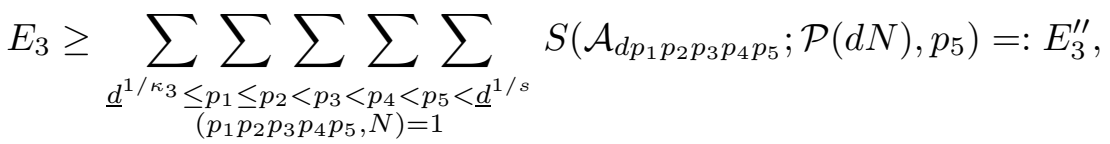

$$
\begin{aligned}
& E_{8} \geq \sum_{\substack{d^{1 / \kappa_{2}} \leq p_{1}<\underline{d}^{1 / \kappa_{3}} \leq p_{2} \leq p_{3}<p_{4}<\underline{d}^{1 / s} \\
\left(p_{1} p_{2} p_{3} p_{4}, N\right)=1}} S\left(\mathcal{A}_{d p_{1} p_{2} p_{3} p_{4}} ; \mathcal{P}(d N), p_{4}\right)=: E_{8}^{\prime} \text {, }
\end{aligned}
$$

and

$$
\begin{aligned}
& D_{6}=\Gamma_{16}+\Gamma_{17}+\Gamma_{18} \\
& +\sum_{\underline{d}^{1 / \kappa_{2}} \leq p_{1}<\underline{d}^{1 / \kappa_{3}} \leq p_{p_{2}<p_{3}<p_{4}<\underline{d}^{1 / s}}} \sum_{\substack{\left.p_{1} p_{2} p_{3} p_{4}, N\right)=1 \\
\underline{p}^{\prime}}} S\left(\mathcal{A}_{d p_{1} p_{2} p_{3} p_{4}} ; \mathcal{P}(d N), p_{3}\right) \\
& +\sum_{\underline{d}^{1 / \kappa} 3 \leq p_{1}<p_{2}<p_{3}<p_{4}<\underline{d}^{1 / s}} \sum_{\substack{\left(p_{1} p_{2} p_{3} p_{4}, N\right)=1 \\
n_{2}}} S\left(\mathcal{A}_{d p_{1} p_{2} p_{3} p_{4}} ; \mathcal{P}(d N), p_{3}\right) \\
& =: \Gamma_{16}+\Gamma_{17}+\Gamma_{18}+D_{6}^{\prime}+D_{6}^{\prime \prime} \text {. }
\end{aligned}
$$

Since

$$
D_{6}^{\prime}-E_{8}^{\prime}=\Gamma_{20}+O_{\delta, k}\left(N^{1-\eta} / d\right)
$$

and

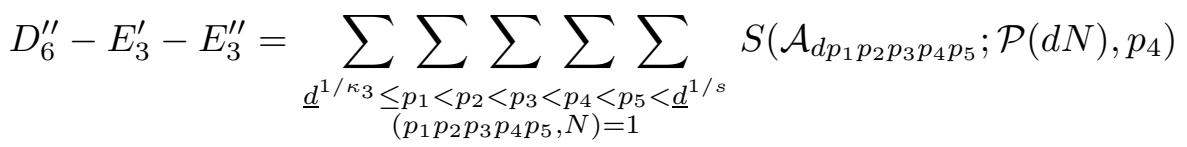




$$
\begin{aligned}
& -\sum_{\substack{d^{1 / \kappa 3} \leq p_{1}<p_{2}<p_{3}<p_{4}<p_{5}<\underline{d}^{1 / s} \\
\left(p_{1} p_{2} p_{3} p_{4} p_{5}, N\right)=1}} S\left(\mathcal{A}_{d p_{1} p_{2} p_{3} p_{4} p_{5}} ; \mathcal{P}(d N), p_{5}\right) \\
& +O_{\delta, k}\left(N^{1-\eta} / d\right) \\
= & \Gamma_{21}+O_{\delta, k}\left(N^{1-\eta} / d\right)
\end{aligned}
$$

we have

$$
\begin{aligned}
D_{6}-2 E_{3}-E_{8} & \leq \Gamma_{16}+\Gamma_{17}+\Gamma_{18}+D_{6}^{\prime}+D_{6}^{\prime \prime}-E_{3}^{\prime}-E_{3}^{\prime \prime}-E_{8}^{\prime} \\
& =\Gamma_{16}+\Gamma_{17}+\Gamma_{18}+\Gamma_{20}+\Gamma_{21}+O_{\delta, k}\left(N^{1-\eta} / d\right) .
\end{aligned}
$$

Combining these estimations leads to the following inequalities:

$$
\begin{aligned}
\Delta_{2} & \leq D_{3}+D_{4}+D_{5}-E_{2}-2 E_{3}-E_{4}-E_{7} \\
& \leq \Gamma_{10}+\cdots+\Gamma_{15}+\Gamma_{19}+D_{6}-2 E_{3}-E_{8}+O_{\delta, k}\left(N^{1-\eta} / d\right) \\
& \leq \Gamma_{10}+\cdots+\Gamma_{21}+O_{\delta, k}\left(N^{1-\eta} / d\right) .
\end{aligned}
$$

Now the desired result follows from (4.12) and (4.13).

5. Functional inequalities between $H(s)$ and $h(s)$. In this section, we start from two weighted inequalities for the sieve function to deduce two functional inequalities between $H(s)$ and $h(s)$. They will be used to prove Propositions 3 and 4 in the next section.

Lemma 5.1. For $5 \geq s^{\prime} \geq 3 \geq s \geq 2$ and $s^{\prime}-s^{\prime} / s \geq 2$, we have

$$
H(s) \geq \Psi_{1}(s)+\frac{1}{2} \int_{1-1 / s}^{1-1 / s^{\prime}} \frac{h\left(s^{\prime} t\right)}{t(1-t)} d t+H\left(s^{\prime}\right),
$$

where $\Psi_{1}(s)=\Psi_{1}\left(s, s^{\prime}\right)$ is given by

$$
\Psi_{1}(s):=-\int_{2}^{s^{\prime}-1} \frac{\log (t-1)}{t} d t+\frac{1}{2} \int_{1-1 / s}^{1-1 / s^{\prime}} \frac{\log \left(s^{\prime} t-1\right)}{t(1-t)} d t-I_{1}(s)
$$

and $I_{1}(s)=I_{1}\left(s, s^{\prime}\right)$ is given by

$$
I_{1}(s):=\max _{\phi \geq 2} \iiint_{1 / s^{\prime} \leq t \leq u \leq v \leq 1 / s} \omega\left(\frac{\phi-t-u-v}{u}\right) \frac{d t d u d v}{t u^{2} v} .
$$

Proof. Our starting point is the inequality in Lemma 4.1. We need to estimate all terms on the right-hand side of this inequality.

Firstly, (3.3) of Lemma 3.2 gives

$$
\sum_{d} \sigma(d) \Omega_{1} \leq 2\left\{A\left(s^{\prime}\right)-H_{k, N_{0}}\left(s^{\prime}\right)\right\} \Theta(N, \sigma) .
$$


Secondly, by an argument similar to the proof of (3.20), we can prove, for any $\varepsilon>0$ and $N \geq N_{0}(\varepsilon, \delta, k)$,

$$
\sum_{d} \sigma(d) \Omega_{2} \geq\left\{\int_{1-1 / s}^{1-1 / s^{\prime}} \frac{a\left(s^{\prime} t\right)+h_{k+1, N_{0}}\left(s^{\prime} t\right)}{t(1-t)} d t-\varepsilon\right\} \Theta(N, \sigma) .
$$

Finally, we apply the switching principle to estimate $\sum_{d} \sigma(d) \Omega_{3}$. For this, we introduce

$$
\mathcal{E}:=\left\{e: e=d n p_{1} p_{2}, \sigma(d) \neq 0,\left(n, p_{1}, p_{2}\right) \text { satisfies (5.3) below }\right\},
$$

where

$$
\left\{\begin{array}{l}
\underline{d}^{1 / s^{\prime}} \leq p_{1}<p_{2}<\underline{d}^{1 / s}, \quad\left(p_{1} p_{2}, d N\right)=1 \\
n \leq N / d p_{1} p_{2}^{2}, \quad\left(n, N P\left(p_{2}\right) / p_{1}\right)=1
\end{array}\right.
$$

and

$$
\mathcal{B}:=\left\{b: b=N-e p_{3}, e \in \mathcal{E}, p_{2}<p_{3} \leq \kappa(d, e)\right\},
$$

where $\kappa(d, e):=\min \left\{N / e, \underline{d}^{1 / s}\right\}$. The set $\mathcal{B}$ is a multiset and an element $b$ may occur more than once.

Clearly $\sum_{d} \sigma(d) \Omega_{3}$ does not exceed the number of primes in the set $\mathcal{B}$. Thus

$$
\sum_{d} \sigma(d) \Omega_{3} \leq S\left(\mathcal{B} ; \mathcal{P}(N), Q^{1 / 2}\right)+O\left(Q^{1 / 2}\right) .
$$

In the set $\mathcal{E}, d$ is not uniquely determined by $e$. This causes technical difficulties. In order to avoid them, we define $\mathcal{E}^{\prime}$ and $\mathcal{B}^{\prime}$, similarly to $\mathcal{E}$ and $\mathcal{B}$, with the condition $\left(n, N P\left(p_{2}\right) / p_{1}\right)=1$ replaced by $\left(n, d N P\left(p_{2}\right) / p_{1}\right)=$ 1 and $\mathcal{E}$ by $\mathcal{E}^{\prime}$ respectively. Obviously the difference $S\left(\mathcal{B} ; \mathcal{P}(N), Q^{1 / 2}\right)-$ $S\left(\mathcal{B}^{\prime} ; \mathcal{P}(N), Q^{1 / 2}\right)$ is

$$
\leq \sum_{d} \sigma(d) \sum_{\underline{d}^{1 / s^{\prime}} \leq p_{1}<p_{2}<p_{3}<\underline{d}^{1 / s}} \sum_{\substack{n \leq N / d p_{1} p_{2} p_{3} \\(n, d)=1}} 1 \ll_{\delta, k} N^{1-\eta},
$$

where $\eta=\eta(\delta, k)>0$. Hence

$$
\sum_{d} \sigma(d) \Omega_{3} \leq S\left(\mathcal{B}^{\prime} ; \mathcal{P}(N), Q^{1 / 2}\right)+O_{\delta, k}\left(N^{1-\eta}\right) .
$$

In order to estimate $S\left(\mathcal{B}^{\prime} ; \mathcal{P}(N), Q^{1 / 2}\right)$, we use Theorem 5.2 in [14] with

$$
X:=\sum_{e \in \mathcal{E}^{\prime}} \sum_{p_{2}<p_{3} \leq \kappa(d, e)} 1, \quad w(q)= \begin{cases}q / \varphi(q) & \text { if } \mu(q)^{2}=(q, N)=1, \\ 0 & \text { otherwise }\end{cases}
$$

to write

$$
S\left(\mathcal{B} ; \mathcal{P}(N), Q^{1 / 2}\right) \leq 8(1+\varepsilon) \frac{C_{N} X}{\log N}+O\left(R_{1}+R_{2}\right),
$$


where $\nu_{*}(q):=\mu(q)^{2} 3^{\nu(q)}$ and

$$
\begin{aligned}
R_{1} & :=\sum_{\substack{q<Q \\
q \mid P\left(Q^{1 / 2}\right)}} \nu_{*}(q)\left|\sum_{\substack{e \in \mathcal{E}^{\prime} \\
(e, q)=1}}\left(\sum_{\substack{p_{2}<p_{3} \leq \kappa(d, e) \\
e p_{3} \equiv N(\bmod q)}} 1-\frac{1}{\varphi(q)} \sum_{p_{2}<p_{3} \leq \kappa(d, e)} 1\right)\right|, \\
R_{2} & :=\sum_{\substack{q<Q \\
q \mid P\left(Q^{1 / 2}\right)}} \frac{3^{\nu(q)}}{\varphi(q)} \sum_{\substack{e \in \mathcal{E}^{\prime} \\
(e, q)>1}} \sum_{p_{2}<p_{3} \leq \kappa(d, e)} 1 . \\
& 1 .
\end{aligned}
$$

We first estimate $R_{2}$. Noticing that for $e \in \mathcal{E}^{\prime}$ we have $e \leq N^{1-\eta}$ and the smallest prime factor of $e$ is $\geq \min \left\{p_{1}, V_{k} / \Delta\right\} \geq W_{k}^{1 / s^{\prime}}$, we deduce that

$$
\begin{aligned}
R_{2} & \leq N \sum_{q \leq Q} \frac{3^{\nu(q)}}{\varphi(q)} \sum_{\substack{e \leq N^{1-\eta} \\
(e, q) \geq W_{k}^{1 / s^{\prime}}}} \frac{1}{e} \\
& \ll N \mathcal{L} \sum_{m \geq W_{k}^{1 / s^{\prime}}} \frac{1}{m} \sum_{\substack{q \leq Q \\
q \equiv 0(\bmod m)}} \frac{3^{\nu(q)}}{\varphi(q)} \\
& \ll N W_{k}^{1 / 3 s^{\prime}} \sum_{m \geq W_{k}^{1 / s^{\prime}}} \frac{1}{m} \sum_{\substack{q \leq Q \\
q \equiv 0(\bmod m)}} \frac{1}{q} \\
& \ll N W_{k}^{1 / 2 s^{\prime}} \sum_{\substack{m \geq W_{k}^{1 / s^{\prime}} \\
m^{2}}} \frac{1}{\mathcal{L}_{\delta, k}} \frac{\Theta(N, \sigma)}{\mathcal{L}^{3}} .
\end{aligned}
$$

Next we estimate $R_{1}$. Let $g(a):=\sum_{e \in \mathcal{E}^{\prime}, e=a} 1$. Obviously for each $e=$ $d n p_{1} p_{2} \in \mathcal{E}^{\prime}$, the integers $d, n, p_{1}, p_{2}$ are pairwise coprime. Therefore they are uniquely determined by $e$. Thus $g(a) \leq 1$ and there are some injections $r_{0}(e)=d$ and $r(e)=p_{2}$. Then we have

$$
R_{1} \ll \sum_{\substack{q \leq Q \\(q, N)=1}} \nu_{*}(q)\left|\sum_{\substack{a \in I(a) \\(a, q)=1}} g(a)\left(\sum_{\substack{p_{2}<p_{3} \leq \kappa(d, a) \\ a p_{3} \equiv N(\bmod q)}} 1-\frac{1}{\varphi(q)} \sum_{p_{2}<p_{3} \leq \kappa(d, a)} 1\right)\right|,
$$

where $I(a):=\left(r_{0}(a) r_{0}(a)^{2 / s^{\prime}}, N / \underline{r}_{0}(a)^{1 / s^{\prime}}\right)$.

Since $\underline{d}^{1 / s^{\prime}}<r(e)<\underline{d}^{1 / s}$ and $\overline{\operatorname{er}(e)} \leq N$, we can write

$$
R_{1} \ll R_{1,1}+R_{1,2}+R_{1,3},
$$

where

$$
R_{1,1}:=\sum_{\substack{q \leq Q \\(q, N)=1}} \nu_{*}(q)\left|\sum_{\substack{a \in I_{1}(a) \\(a, q)=1}} g(a)\left(\sum_{\substack{p_{3} \leq r_{0}(a) \\ a p_{3} \equiv N(\bmod q)}} 1-\frac{1}{\varphi(q)} \sum_{p_{3} \leq{\underline{r_{0}(a)^{1 / s}}}^{1 / s}} 1\right)\right|,
$$




$$
\begin{aligned}
R_{1,2} & :=\sum_{\substack{q \leq Q \\
(q, N)=1}} \nu_{*}(q)\left|\sum_{\substack{a \in I_{2}(a) \\
(a, q)=1}} g(a)\left(\sum_{\substack{q \leq N / a \\
p_{3} \leq N / a \\
a p_{3} \equiv N(\bmod q)}} 1-\frac{1}{\varphi(q)} \sum_{p_{3} \leq N / a} 1\right)\right|, \\
R_{1,3} & :=\sum_{\substack{q \leq Q \\
q, N)=1}} \nu_{*}(q) \mid \sum_{\substack{a \in I(a) \\
(a, q)=1}} g(a)\left(\sum_{\substack{p_{3} \leq r(a) \\
a p_{3} \equiv N(\bmod q)}} 1-\frac{1}{\varphi(q)} \sum_{p_{3} \leq r(a)} 1\right),
\end{aligned}
$$

and

$$
\begin{aligned}
& I_{1}(a):=\left(r_{0}(a){\underline{r_{0}(a)^{2 / s^{\prime}}}}^{1 / s},{\underline{r_{0}(a)^{1 / s}}}^{1 / s}, N /{\underline{r_{0}(a)^{1 / s^{\prime}}}}^{1 / 2} .\right.
\end{aligned}
$$

Applying Lemma 2.3 yields $R_{1, j} \ll \delta, k N / \mathcal{L}^{5 k+5}$ for $j=1,2,3$. Hence

$$
R_{1} \ll \Theta(N, \sigma) / \mathcal{L}^{3} .
$$

If we replace $\left(n, N P\left(p_{2}\right) / p_{1}\right)=1$ by $\left(n, N P\left(p_{2}\right)\right)=1$ in the definition of $X$, the difference is $\ll_{\delta, k} N \mathcal{L}^{2} / d \underline{d}^{1 / s^{\prime}} \ll_{\delta, k} N / \mathcal{L}^{2} d$. Thus we deduce, by Lemma 2.10, that

$$
\begin{aligned}
X & =\sum_{d} \sigma(d)\left\{\sum_{\underline{d}^{1 / s^{\prime}} \leq p_{1}<p_{2}<p_{3}<\underline{d}^{1 / s}} \sum_{\substack {\left(p_{1} p_{2}, d N\right)=1 \\
\begin{subarray}{c}{n \leq N / d p_{1} p_{2} p_{3} \\
\left(n, N P\left(p_{2}\right)\right)=1{ ( p _ { 1 } p _ { 2 } , d N ) = 1 \\
\begin{subarray} { c } { n \leq N / d p _ { 1 } p _ { 2 } p _ { 3 } \\
( n , N P ( p _ { 2 } ) ) = 1 } }\end{subarray}} 1+O_{\delta, k}\left(N / \mathcal{L}^{2} d\right)\right\} \\
\leq & \left.(1+\varepsilon) \sum_{d} \sigma(d)\left\{\sum_{\underline{d}^{1 / s^{\prime} \leq p_{1}<p_{2}<p_{3}<\underline{d}^{1 / s}}} \sum_{\substack{N \omega\left(\log \left(N / d p_{1} p_{2} p_{3}\right) / \log p_{2}\right) \\
d p_{1} p_{2} p_{3} \log p_{2}}} \frac{N \omega .}{\mathcal{L}^{2} d}\right)\right\} .
\end{aligned}
$$

By applying the prime number theorem, we obtain

$$
\left.X \leq(1+\varepsilon) N \sum_{d} \frac{\sigma(d)}{d \log \underline{d}_{1 / s^{\prime} \leq t \leq u \leq v \leq 1 / s}} \iiint_{u} \frac{\phi_{d, N}-t-u-v}{u}\right) \frac{d t d u d v}{t u^{2} v},
$$

where $\phi_{d, N}:=\log (N / d) / \log \underline{d}$. Obviously $\sigma(d) \neq 0$ implies $\phi_{d, N} \geq 2$. Thus

$$
X \leq(1+\varepsilon) I_{1}(s) N \sum_{d} \frac{\sigma(d)}{d \log \underline{d}} \leq(1+\varepsilon) I_{1}(s) N \sum_{d} \frac{\sigma(d)}{\varphi(d) \log \underline{d}} .
$$

Combining (5.4)-(5.8) and noticing $C_{N} \leq C_{d N}$, we obtain, for any $\varepsilon>0$ and $N \geq N_{0}(\varepsilon, \delta, k)$

$$
\sum_{d} \sigma(d) \Omega_{3} \leq\left\{2 I_{1}(s)+\varepsilon\right\} \Theta(N, \sigma)
$$

Inserting (5.1), (5.2) and (5.9) into the inequality of Lemma 4.1 and noticing 
that

$$
A\left(s^{\prime}\right)=A(s)+\int_{2}^{s^{\prime}-1} \frac{\log (t-1)}{t} d t, \quad a\left(s^{\prime} t\right)=\log \left(s^{\prime} t-1\right),
$$

we find that

$$
\begin{aligned}
\Phi(N, \sigma, s) \leq & \left\{A(s)-\Psi_{1}(s)-\frac{1}{2} \int_{1-1 / s}^{1-1 / s^{\prime}} \frac{h_{k+1, N_{0}}\left(s^{\prime} t\right)}{t(1-t)} d t\right. \\
& \left.-H_{k, N_{0}}\left(s^{\prime}\right)+\varepsilon\right\} \Theta(N, \sigma) .
\end{aligned}
$$

By the definition of $H_{k, N_{0}}(s)$, we must have

$$
H_{k, N_{0}}(s) \geq \Psi_{1}(s)+\frac{1}{2} \int_{1-1 / s}^{1-1 / s^{\prime}} \frac{h_{k+1, N_{0}}\left(s^{\prime} t\right)}{t(1-t)} d t+H_{k, N_{0}}\left(s^{\prime}\right)-\varepsilon .
$$

Letting $N_{0} \rightarrow \infty$ and then $\varepsilon \rightarrow 0$ yields

$$
H_{k}(s) \geq \Psi_{1}(s)+\frac{1}{2} \int_{1-1 / s}^{1-1 / s^{\prime}} \frac{h_{k+1}\left(s^{\prime} t\right)}{t(1-t)} d t+H_{k}\left(s^{\prime}\right) .
$$

Now it remains to let $k \rightarrow \infty$ to get the desired result.

Lemma 5.2. For $5 \geq s^{\prime} \geq 3 \geq s \geq 2, s^{\prime}-s^{\prime} / s \geq 2$ and $s \leq \kappa_{3}<\kappa_{2}<$ $\kappa_{1} \leq s^{\prime}$, we have

$$
\begin{aligned}
H(s) \geq & \Psi_{2}(s)+\frac{4}{5} H\left(s^{\prime}\right)+\frac{1}{5} H\left(\kappa_{1}\right)+\frac{1}{5} \int_{1-1 / s}^{1-1 / s^{\prime}} \frac{h\left(s^{\prime} t\right)}{t(1-t)} d t \\
& +\frac{1}{5} \int_{1-1 / \kappa_{2}}^{1-1 / s^{\prime}} \frac{h\left(s^{\prime} t\right)}{t(1-t)} d t+\frac{1}{5} \int_{1-1 / \kappa_{3}}^{1-1 / s^{\prime}} \frac{h\left(s^{\prime} t\right)}{t(1-t)} d t \\
& +\frac{1}{5} \int_{1 / s^{\prime}}^{1 / \kappa_{2}} \frac{d t}{t} \int_{t}^{1 / \kappa_{2}} \frac{H\left(s^{\prime}-s^{\prime} t-s^{\prime} u\right)}{u(1-t-u)} d u \\
& +\frac{1}{5} \int_{1 / s^{\prime}}^{1 / \kappa_{1}} \frac{d t}{t} \int_{1 / \kappa_{2}}^{1 / \kappa_{3}} \frac{H\left(s^{\prime}-s^{\prime} t-s^{\prime} u\right)}{u(1-t-u)} d u \\
& +\frac{1}{5} \int_{1 / s^{\prime}}^{1 / \kappa_{1}} \frac{d t}{t} \int_{t}^{1 / \kappa_{2}} \frac{H((1-t-u) / t)}{u(1-t-u)} d u
\end{aligned}
$$


where $\Psi_{2}(s)=\Psi_{2}\left(s, s^{\prime}, \kappa_{1}, \kappa_{2}, \kappa_{3}\right)$ is given by

$$
\begin{aligned}
\Psi_{2}(s):= & -\frac{2}{5} \int_{2}^{s^{\prime}-1} \frac{\log (t-1)}{t} d t-\frac{2}{5} \int_{2}^{\kappa_{1}-1} \frac{\log (t-1)}{t} d t \\
& -\frac{1}{5} \int_{2}^{\kappa_{2}-1} \frac{\log (t-1)}{t} d t+\frac{1}{5} \int_{1-1 / s}^{1-1 / s^{\prime}} \frac{\log \left(s^{\prime} t-1\right)}{t(1-t)} d t \\
& +\frac{1}{5} \int_{1-1 / \kappa_{3}}^{1-1 / \kappa_{1}} \frac{\log \left(\kappa_{1} t-1\right)}{t(1-t)} d t-\frac{2}{5} \sum_{i=9}^{21} I_{2, i}(s)
\end{aligned}
$$

and $I_{2, i}(s)=I_{2, i}\left(s, s^{\prime}, \kappa_{1}, \kappa_{2}, \kappa_{3}\right)$ is given by

$$
\begin{aligned}
I_{2, i}(s) & :=\max _{\phi \geq 2} \int_{\mathbb{D}_{2, i}} \omega\left(\frac{\phi-t-u-v}{u}\right) \frac{d t d u d v}{t u^{2} v} \quad(9 \leq i \leq 15), \\
I_{2, i}(s) & :=\max _{\phi \geq 2} \int_{\mathbb{D}_{2, i}} \omega\left(\frac{\phi-t-u-v-w}{v}\right) \frac{d t d u d v d w}{t u v^{2} w} \quad(16 \leq i \leq 19), \\
I_{2,20}(s) & :=\max _{\phi \geq 2} \int_{\mathbb{D}_{2,20}} \omega\left(\frac{\phi-t-u-v-w-x}{w}\right) \frac{d t d u d v d w d x}{t u v w^{2} x}, \\
I_{2,21}(s) & :=\max _{\phi \geq 2} \int_{\mathbb{D}_{2,21}} \omega\left(\frac{\phi-t-u-v-w-x-y}{x}\right) \frac{d t d u d v d w d x d y}{t u v w x^{2} y} .
\end{aligned}
$$

The sets $\mathbb{D}_{2, i}(9 \leq i \leq 21)$ are defined as follows:

$$
\begin{aligned}
& \mathbb{D}_{2,9}:=\left\{(t, u, v): 1 / \kappa_{1} \leq t \leq u \leq v \leq 1 / \kappa_{3}\right\} \\
& \mathbb{D}_{2,10}:=\left\{(t, u, v): 1 / \kappa_{1} \leq t \leq u \leq 1 / \kappa_{2} \leq v \leq 1 / s\right\} \\
& \mathbb{D}_{2,11}:=\left\{(t, u, v): 1 / \kappa_{1} \leq t \leq 1 / \kappa_{2} \leq u \leq v \leq 1 / \kappa_{3}\right\} \\
& \mathbb{D}_{2,12}:=\left\{(t, u, v): 1 / s^{\prime} \leq t \leq u \leq 1 / \kappa_{1}, 1 / \kappa_{3} \leq v \leq 1 / s\right\} \\
& \mathbb{D}_{2,13}:=\left\{(t, u, v): 1 / s^{\prime} \leq t \leq 1 / \kappa_{1} \leq u \leq 1 / \kappa_{2} \leq v \leq 1 / s\right\} \\
& \mathbb{D}_{2,14}:=\left\{(t, u, v): 1 / s^{\prime} \leq t \leq 1 / \kappa_{1}, 1 / \kappa_{2} \leq u \leq v \leq 1 / s\right\} \\
& \mathbb{D}_{2,15}:=\left\{(t, u, v): 1 / \kappa_{1} \leq t \leq 1 / \kappa_{2} \leq u \leq 1 / \kappa_{3} \leq v \leq 1 / s\right\} \\
& \mathbb{D}_{2,16}:=\left\{(t, u, v, w): 1 / \kappa_{2} \leq t \leq u \leq v \leq w \leq 1 / \kappa_{3}\right\} \\
& \mathbb{D}_{2,17}:=\left\{(t, u, v, w): 1 / \kappa_{2} \leq t \leq u \leq v \leq 1 / \kappa_{3} \leq w \leq 1 / s\right\} \\
& \mathbb{D}_{2,18}:=\left\{(t, u, v, w): 1 / \kappa_{2} \leq t \leq u \leq 1 / \kappa_{3} \leq v \leq w \leq 1 / s\right\} \\
& \mathbb{D}_{2,19}:=\left\{(t, u, v, w): 1 / \kappa_{1} \leq t \leq 1 / \kappa_{2}, 1 / \kappa_{3} \leq u \leq v \leq w \leq 1 / s\right\} \\
& \mathbb{D}_{2,20}:=\left\{(t, u, v, w, x): 1 / \kappa_{2} \leq t \leq 1 / \kappa_{3} \leq u \leq v \leq w \leq x \leq 1 / s\right\} \\
& \mathbb{D}_{2,21}:=\left\{(t, u, v, w, x, y): 1 / \kappa_{3} \leq t \leq u \leq v \leq w \leq x \leq y \leq 1 / s\right\} .
\end{aligned}
$$


Proof. By (3.3) of Lemma 3.2, we have

$$
\sum_{d} \sigma(d) \Gamma_{1} \leq\left\{4 A\left(s^{\prime}\right)+A\left(\kappa_{1}\right)-4 H_{k, N_{0}}\left(s^{\prime}\right)-H_{k, N_{0}}\left(\kappa_{1}\right)\right\} \Theta(N, \sigma) .
$$

Similarly to (3.20), we can prove

$$
\sum_{d} \sigma(d) \Gamma_{2} \geq\left\{\int_{1-1 / s}^{1-1 / s^{\prime}} \frac{a\left(s^{\prime} t\right)+h_{k+1, N_{0}}\left(s^{\prime} t\right)}{t(1-t)} d t-\varepsilon\right\} \Theta(N, \sigma)
$$

$$
\sum_{d} \sigma(d) \Gamma_{3} \geq\left\{\int_{1-1 / \kappa_{2}}^{1-1 / s^{\prime}} \frac{a\left(s^{\prime} t\right)+h_{k+1, N_{0}}\left(s^{\prime} t\right)}{t(1-t)} d t-\varepsilon\right\} \Theta(N, \sigma)
$$

$$
\sum_{d} \sigma(d) \Gamma_{4} \geq\left\{\int_{1-1 / \kappa_{3}}^{1-1 / s^{\prime}} \frac{a\left(s^{\prime} t\right)+h_{k+1, N_{0}}\left(s^{\prime} t\right)}{t(1-t)} d t-\varepsilon\right\} \Theta(N, \sigma) .
$$

Similarly to (3.20) and in view of $A\left(s^{\prime}-s^{\prime} t-s^{\prime} u\right)=A((1-t-u) / t)=1$, we have

$$
\begin{aligned}
\sum_{d} \sigma(d) & \Gamma_{5} \\
& \leq\left\{\int_{1 / s^{\prime}}^{1 / \kappa_{2}} \frac{d t}{t} \int_{t}^{1 / \kappa_{2}} \frac{1-H_{k+2, N_{0}}\left(s^{\prime}-s^{\prime} t-s^{\prime} u\right)}{u(1-t-u)} d u+\varepsilon\right\} \Theta(N, \sigma),
\end{aligned}
$$

$$
\begin{aligned}
\sum_{d} \sigma(d) & \Gamma_{6} \\
& \leq\left\{\int_{1 / s^{\prime}}^{1 / \kappa_{1}} \frac{d t}{t} \int_{1 / \kappa_{2}}^{1 / \kappa_{3}} \frac{1-H_{k+2, N_{0}}\left(s^{\prime}-s^{\prime} t-s^{\prime} u\right)}{u(1-t-u)} d u+\varepsilon\right\} \Theta(N, \sigma),
\end{aligned}
$$

$$
\begin{aligned}
\sum_{d} \sigma(d) & \Gamma_{7} \\
& \leq\left\{\int_{1 / s^{\prime}}^{1 / \kappa_{1}} \frac{d t}{t} \int_{t}^{1 / \kappa_{1}} \frac{1-H_{k+2, N_{0}}((1-t-u) / t)}{u(1-t-u)} d u+\varepsilon\right\} \Theta(N, \sigma),
\end{aligned}
$$

$$
\begin{aligned}
\sum_{d} \sigma(d) & \Gamma_{8} \\
& \leq\left\{\int_{1 / s^{\prime}}^{1 / \kappa_{1}} \frac{d t}{t} \int_{1 / \kappa_{1}}^{1 / \kappa_{2}} \frac{1-H_{k+2, N_{0}}((1-t-u) / t)}{u(1-t-u)} d u+\varepsilon\right\} \Theta(N, \sigma) .
\end{aligned}
$$

We also have, for $i=9, \ldots, 21$,

$$
\sum_{d} \sigma(d) \Gamma_{i} \leq\left\{2 I_{2, i}(s)+\varepsilon\right\} \Theta(N, \sigma) .
$$


As before, inserting (5.11)-(5.19) into the inequality of Lemma 4.2 and using the definition of $H_{k, N_{0}}(s)$, we deduce that

$$
5 H_{k, N_{0}}(s) \geq A\left(s, s^{\prime}\right)+B\left(s, s^{\prime}\right)-\varepsilon,
$$

where

$$
\begin{aligned}
A\left(s, s^{\prime}\right):= & 5 A(s)-4 A\left(s^{\prime}\right)-A\left(\kappa_{1}\right)+4 H_{k, N_{0}}\left(s^{\prime}\right)+H_{k, N_{0}}\left(\kappa_{1}\right) \\
& +\int_{1-1 / s}^{1-1 / s^{\prime}} \frac{a\left(s^{\prime} t\right)}{t(1-t)} d t+\int_{1-1 / \kappa_{2}}^{1-1 / s^{\prime}} \frac{a\left(s^{\prime} t\right)}{t(1-t)} d t \\
& +\int_{1-1 / \kappa_{3}}^{1-1 / s^{\prime}} \frac{a\left(s^{\prime} t\right)}{t(1-t)} d t-\int_{1 / s^{\prime}}^{1 / \kappa_{2}} \int_{t}^{1 / \kappa_{2}} \frac{d t d u}{t u(1-t-u)} \\
& -\int_{1 / s^{\prime}}^{1 / \kappa_{1} 1 / \kappa_{3}} \int_{t}^{1 u(1-t-u)}-2 \sum_{i=9}^{21} I_{2, i}(s)
\end{aligned}
$$

and

$$
\begin{aligned}
B\left(s, s^{\prime}\right):= & \left(\int_{1-1 / s}^{1-1 / s^{\prime}}+\int_{1-1 / \kappa_{2}}^{1-1 / s^{\prime}}+\int_{1-1 / \kappa_{3}}^{1-1 / s^{\prime}}\right) \frac{h_{k+1, N_{0}}\left(s^{\prime} t\right)}{t(1-t)} d t \\
& +\int_{1 / s^{\prime}}^{1 / \kappa_{2}} \frac{d t}{t} \int_{t}^{1 / \kappa_{2}} \frac{H_{k+2, N_{0}}\left(s^{\prime}-s^{\prime} t-s^{\prime} u\right)}{u(1-t-u)} d u \\
& +\int_{1 / s^{\prime}}^{1 / \kappa_{1}} \frac{d t}{t} \int_{1 / \kappa_{2}}^{1 / \kappa_{3}} \frac{H_{k+2, N_{0}}\left(s^{\prime}-s^{\prime} t-s^{\prime} u\right)}{u(1-t-u)} d u \\
& +\int_{1 / s^{\prime}}^{1 / \kappa_{1}} \frac{d t}{t} \int_{t}^{1 / \kappa_{2}} \frac{H_{k+2, N_{0}}((1-t-u) / t)}{u(1-t-u)} d u .
\end{aligned}
$$

For $a \geq b>2$, we have

$$
\begin{aligned}
\int_{1 / a}^{1 / b} \frac{d t}{t} \int_{t}^{1 / b} \frac{d u}{u(1-t-u)} & =\int_{1 / a}^{1 / b} \frac{d u}{u} \int_{1 / a}^{u} \frac{d t}{t(1-t-u)} \\
& =\int_{1 / a}^{1 / b} \frac{\log (a-1-a u)-\log (1 / u-2)}{u(1-u)} d u \\
& =\int_{1-1 / b}^{1-1 / a} \frac{\log (a t-1)}{t(1-t)} d t-\int_{b-1}^{a-1} \frac{\log (t-1)}{t} d t
\end{aligned}
$$

where we have used the change of variables $t=1-u$ and $t=1 / u-1$ respectively. 
Similarly for $a \geq b \geq c \geq d>2$, we have

$$
\int_{1 / a}^{1 / b} \frac{d t}{t} \int_{1 / c}^{1 / d} \frac{d u}{u(1-t-u)}=\int_{1-1 / d}^{1-1 / c} \frac{\log (a t-1)}{t(1-t)} d t-\int_{1-1 / d}^{1-1 / c} \frac{\log (b t-1)}{t(1-t)} d t .
$$

By these two relations and (5.10), a simple calculation shows

$$
A\left(s, s^{\prime}\right)=5 \Psi_{2}(s)+4 H_{k, N_{0}}\left(s^{\prime}\right)+H_{k, N_{0}}\left(\kappa_{1}\right) .
$$

Inserting this into (5.20) and letting $N \rightarrow \infty, \varepsilon \rightarrow 0$ and $k \rightarrow \infty$, we obtain the desired inequality. This completes the proof.

6. Proofs of Propositions 3 and 4. We first prove a preliminary lemma. Let $\mathbf{1}_{[a, b]}(t)$ be the characteristic function of the interval $[a, b]$. We recall that

$$
\sigma(a, b, c):=\int_{a}^{b} \log \frac{c}{t-1} \frac{d t}{t}, \quad \sigma_{0}(t):=\frac{\sigma(3, t+2, t+1)}{1-\sigma(3,5,4)} .
$$

Lemma 6.1. Let $3 \leq s^{\prime} \leq 5,0<a<b<1$ and $2 \leq a c<b c \leq 4$. Then

$$
\begin{gathered}
h(4) \geq \int_{1}^{3} H(t) \frac{\sigma_{0}(t)}{t} d t \\
H\left(s^{\prime}\right) \geq \int_{1}^{3} H(t)\left\{\frac{\sigma_{0}(t)}{t} \log \left(\frac{4}{s^{\prime}-1}\right)\right. \\
\left.+\frac{\mathbf{1}_{\left[s^{\prime}-2,3\right]}(t)}{t} \log \left(\frac{t+1}{s^{\prime}-1}\right)\right\} d t \\
\int_{a}^{b} \frac{h(c t)}{t(1-t)} d t \geq \log \left(\frac{b-a b}{a-a b}\right) \int_{1}^{3} H(t) \frac{\sigma_{0}(t)+\mathbf{1}_{[b c-1,3]}(t)}{t} d t \\
+\int_{1}^{3} H(t) \frac{\mathbf{1}_{[a c-1, b c-1]}(t)}{t} \log \left(\frac{(1-a)(t+1)}{a(c-1-t)}\right) d t .
\end{gathered}
$$

Proof. By Proposition 2, we have

$$
\begin{aligned}
H\left(s^{\prime}\right) & \geq \int_{s^{\prime}-1}^{4} \frac{h(u)}{u} d u \geq \int_{s^{\prime}-1}^{4}\left(h(4)+\int_{u-1}^{3} H(t) \frac{d t}{t}\right) \frac{d u}{u} \\
& =h(4) \log \left(\frac{4}{s^{\prime}-1}\right)+\int_{1}^{3} H(t) \frac{\mathbf{1}_{\left[s^{\prime}-2,3\right]}(t)}{t} \log \left(\frac{t+1}{s^{\prime}-1}\right) d t
\end{aligned}
$$


From Proposition 2 and (6.4), we deduce that

$$
h(4) \geq \int_{3}^{5} \frac{H(v)}{v} d v \geq h(4) \sigma(3,5,4)+\int_{1}^{3} H(t) \frac{\sigma(3, t+2, t+1)}{t} d t,
$$

which implies the inequality (6.1).

The inequality (6.2) follows immediately from (6.4) and (6.1).

By Proposition 2, we have

$$
\begin{aligned}
\int_{a}^{b} \frac{h(c t)}{t(1-t)} d t & =c \int_{a c}^{b c} \frac{h(u)}{u(c-u)} d u \\
\geq & c \int_{a c}^{b c} \frac{d u}{u(c-u)}\left(h(4)+\int_{u-1}^{3} H(t) \frac{d t}{t}\right) \\
= & \left\{h(4)+\int_{1}^{3} H(t) \frac{\mathbf{1}_{[b c-1,3]}(t)}{t} d t\right\} \log \left(\frac{b-a b}{a-a b}\right) \\
& +\int_{1}^{3} H(t) \frac{\mathbf{1}_{[a c-1, b c-1]}(t)}{t} \log \left(\frac{(1-a)(t+1)}{a(c-1-t)}\right) d t
\end{aligned}
$$

combining this with (6.1) gives (6.3). This completes the proof.

Proof of Proposition 3. By Lemma 6.1, a simple calculation shows

$$
\frac{1}{2} \int_{1-1 / s}^{1-1 / s^{\prime}} \frac{h\left(s^{\prime} t\right)}{t(1-t)} d t+H\left(s^{\prime}\right) \geq \int_{1}^{3} H(t) \Xi_{1}(t ; s) d t,
$$

which, together with Lemma 4.1, implies the desired result.

Proof of Proposition 4. From (6.1)-(6.3), we deduce that

$$
\begin{aligned}
& \int_{1-1 / s}^{1-1 / s^{\prime}} \frac{h\left(s^{\prime} t\right)}{t(1-t)} d t+\int_{1-1 / \kappa_{2}}^{1-1 / s^{\prime}} \frac{h\left(s^{\prime} t\right)}{t(1-t)} d t+\int_{1-1 / \kappa_{3}}^{1-1 / s^{\prime}} \frac{h\left(s^{\prime} t\right)}{t(1-t)} d t \\
& \quad+4 H\left(s^{\prime}\right)+H\left(\kappa_{1}\right) \\
& \geq \int_{1}^{3} H(t) \frac{\mathbf{1}_{\left[\alpha_{2}, 3\right]}(t)}{t} \log \left(\frac{(t+1)^{5}}{(s-1)\left(s^{\prime}-1\right)\left(\kappa_{1}-1\right)\left(\kappa_{2}-1\right)\left(\kappa_{3}-1\right)}\right) d t \\
& \quad+\int_{1}^{3} H(t) \frac{\sigma_{0}(t)}{t} \log \left(\frac{1024}{(s-1)\left(s^{\prime}-1\right)\left(\kappa_{1}-1\right)\left(\kappa_{2}-1\right)\left(\kappa_{3}-1\right)}\right) d t
\end{aligned}
$$




$$
\begin{aligned}
& +\int_{1}^{3} H(t) \frac{\mathbf{1}_{\left[\alpha_{5}, \alpha_{2}\right]}(t)}{t} \log \left(\frac{t+1}{\left(\kappa_{3}-1\right)\left(s^{\prime}-1-t\right)}\right) d t \\
& +\int_{1}^{3} H(t) \frac{\mathbf{1}_{\left[\alpha_{4}, \alpha_{2}\right]}(t)}{t} \log \left(\frac{t+1}{\left(\kappa_{2}-1\right)\left(s^{\prime}-1-t\right)}\right) d t \\
& +\int_{1}^{3} H(t) \frac{\mathbf{1}_{\left[\alpha_{3}, \alpha_{2}\right]}(t)}{t} \log \left(\frac{t+1}{(s-1)\left(s^{\prime}-1-t\right)}\right) d t \\
& +\int_{1}^{3} H(t) \frac{\mathbf{1}_{\left[\alpha_{1}, \alpha_{2}\right]}(t)}{t} \log \left(\frac{t+1}{\kappa_{1}-1}\right) d t .
\end{aligned}
$$

By the change of variable $v=s^{\prime}(1-t-u)$, we have

$$
\begin{aligned}
\int_{1 / s^{\prime}}^{1 / \kappa_{2}} \frac{d t}{t} \int_{t}^{1 / \kappa_{2}} \frac{H\left(s^{\prime}-s^{\prime} t-s^{\prime} u\right)}{u(1-t-u)} & d u \\
& =\int_{1 / s^{\prime}}^{1 / \kappa_{2}} \frac{d t}{t} \int_{s^{\prime}\left(1-1 / \kappa_{2}-t\right)}^{s^{\prime}(1-2 t)} \frac{s^{\prime} H(v)}{v\left(s^{\prime}-s^{\prime} t-v\right)} d v .
\end{aligned}
$$

Interchanging the order of integration and a simple calculation show that

$$
\begin{aligned}
& \int_{1 / s^{\prime}}^{1 / \kappa_{2}} \frac{d t}{t} \int_{t}^{1 / \kappa_{2}} \frac{H\left(s^{\prime}-s^{\prime} t-s^{\prime} u\right)}{u(1-t-u)} d u \\
&=\int_{1}^{3} H(t)\left\{\frac{\mathbf{1}_{\left[\alpha_{6}, \alpha_{4}\right]}(t)}{t\left(1-t / s^{\prime}\right)} \log \left(\frac{s^{\prime}}{\kappa_{2} s^{\prime}-s^{\prime}-\kappa_{2} t}\right)\right. \\
&\left.+\frac{\mathbf{1}_{\left[\alpha_{4}, \alpha_{2}\right]}(t)}{t\left(1-t / s^{\prime}\right)} \log \left(s^{\prime}-1-t\right)\right\} d t
\end{aligned}
$$

Similarly we can prove

$$
\int_{1 / s^{\prime}}^{1 / \kappa_{1}} \frac{d t}{t} \int_{1 / \kappa_{2}}^{1 / \kappa_{3}} \frac{H\left(s^{\prime}-s^{\prime} t-s^{\prime} u\right)}{u(1-t-u)} d u=\int_{1}^{3} H(t) L_{1}(t) d t
$$

where

$$
\begin{aligned}
L_{1}(t):= & \frac{\mathbf{1}_{\left[\alpha_{7}, \alpha_{5}\right]}(t)}{t\left(1-t / s^{\prime}\right)} \log \left(\frac{s^{2}}{\left(\kappa_{1} s^{\prime}-s^{\prime}-\kappa_{1} t\right)\left(\kappa_{3} s^{\prime}-s^{\prime}-\kappa_{3} t\right)}\right) \\
& +\frac{\mathbf{1}_{\left[\alpha_{5}, \alpha_{8}\right]}(t)}{t\left(1-t / s^{\prime}\right)} \log \left(\frac{s^{\prime}\left(s^{\prime}-1-t\right)}{\kappa_{1} s^{\prime}-s^{\prime}-\kappa_{1} t}\right) \\
& +\frac{\mathbf{1}_{\left[\alpha_{8}, \alpha_{4}\right]}(t)}{t\left(1-t / s^{\prime}\right)} \log \left(\frac{\left(s^{\prime}-1-t\right)\left(\kappa_{2} s^{\prime}-s^{\prime}-\kappa_{2} t\right)}{s^{\prime}}\right)
\end{aligned}
$$


and

$$
\int_{1 / s^{\prime}}^{1 / \kappa_{1}} \frac{d t}{t} \int_{t}^{1 / \kappa_{2}} \frac{H((1-t-u) / t)}{u(1-t-u)} d u=\int_{1}^{3} H(t) L_{2}(t) d t
$$

with

$$
\begin{aligned}
L_{2}(t):= & \frac{\mathbf{1}_{\left[\alpha_{9}, \alpha_{1}\right]}(t)}{t} \log \left(\frac{t+1}{\left(\kappa_{2}-1\right)\left(\kappa_{1}-1-t\right)}\right) \\
& +\frac{\mathbf{1}_{\left[\alpha_{1}, \alpha_{4}\right]}(t)}{t} \log \left(\frac{t+1}{\kappa_{2}-1}\right)+\frac{\mathbf{1}_{\left[\alpha_{4}, \alpha_{2}\right]}(t)}{t} \log \left(s^{\prime}-1-t\right) .
\end{aligned}
$$

Now by inserting (6.5)-(6.8) into Lemma 5.2, we easily deduce the required result.

7. Proof of Theorem 1. We need to resolve the functional inequalities (3.21) and (3.22). It seems very difficult to give the exact solutions, because we only know that $H(s)$ is decreasing. Next we shall give a numerical lower bound for solution by a discretization, which is sufficient to prove Theorem 1.

Put $s_{0}:=1$ and $s_{i}:=2+0.1 \cdot(i+1)$ for $i=1, \ldots, 9$. Since $H(s)$ is decreasing on $[1,10]$, Proposition 4 allows us to deduce

$$
H\left(s_{i}\right) \geq \Psi_{2}\left(s_{i}\right)+\sum_{j=1}^{9} a_{i, j} H\left(s_{j}\right),
$$

where

$$
a_{i, j}:=\int_{s_{j-1}}^{s_{j}} \Xi_{2}\left(t, s_{i}\right) d t \quad(i=1, \ldots, 4 ; j=1, \ldots, 9) .
$$

Similarly Proposition 3 implies

$$
H\left(s_{i}\right) \geq \Psi_{1}\left(s_{i}\right)+\sum_{j=1}^{9} a_{i, j} H\left(s_{j}\right)
$$

where

$$
a_{i, j}:=\int_{s_{j-1}}^{s_{j}} \Xi_{1}\left(t, s_{i}\right) d t \quad(i=5, \ldots, 9 ; j=1, \ldots, 9)
$$


Table 1. Choice of parameters

\begin{tabular}{cccccccc}
\hline$i$ & $s_{i}$ & $s_{i}^{\prime}$ & $\kappa_{1, i}$ & $\kappa_{2, i}$ & $\kappa_{3, i}$ & $\Psi_{1}\left(s_{i}\right)$ & $\Psi_{2}\left(s_{i}\right)$ \\
\hline 1 & 2.2 & 4.54 & 3.53 & 2.90 & 2.44 & & 0.015826357 \\
2 & 2.3 & 4.50 & 3.54 & 2.88 & 2.43 & & 0.015247971 \\
3 & 2.4 & 4.46 & 3.57 & 2.87 & 2.40 & & 0.013898757 \\
4 & 2.5 & 4.12 & 3.56 & 2.91 & 2.50 & & 0.011776059 \\
5 & 2.6 & 3.58 & & & & 0.009405211 & \\
6 & 2.7 & 3.47 & & & & 0.006558950 & \\
7 & 2.8 & 3.34 & & & & 0.003536751 & \\
8 & 2.9 & 3.19 & & & & 0.001056651 & \\
9 & 3.0 & 3.00 & & & & 0.000000000 & \\
\hline
\end{tabular}

The parameters $s_{i}^{\prime}, \kappa_{1, i}, \kappa_{2, i}$ and $\kappa_{3, i}$ are chosen such that $\Psi_{1}\left(s_{i}\right)$ or $\Psi_{2}\left(s_{i}\right)$ is maximal.

We put

$$
\mathbf{A}:=\left(\begin{array}{ccc}
a_{1,1} & \cdots & a_{1,9} \\
\vdots & & \vdots \\
a_{9,1} & \cdots & a_{9,9}
\end{array}\right), \quad \mathbf{H}:=\left(\begin{array}{c}
H\left(s_{1}\right) \\
\vdots \\
H\left(s_{9}\right)
\end{array}\right), \quad \mathbf{B}:=\left(\begin{array}{c}
\Psi_{2}\left(s_{1}\right) \\
\vdots \\
\Psi_{2}\left(s_{4}\right) \\
\Psi_{1}\left(s_{5}\right) \\
\vdots \\
\Psi_{1}\left(s_{9}\right)
\end{array}\right) .
$$

Then (7.1) and (7.2) can be written as

$$
(\mathbf{I}-\mathbf{A}) \mathbf{H} \geq \mathbf{B},
$$

where $\mathbf{I}$ is the unit matrix.

In order to resolve (7.3), we first solve the system of linear equations

$$
(\mathbf{I}-\mathbf{A}) \mathbf{X}=\mathbf{B}
$$

by using Maple and obtain

$$
\mathbf{X}=\left(\begin{array}{c}
0.0223939 \ldots \\
0.0217196 \ldots \\
0.0202876 \ldots \\
0.0181433 \ldots \\
0.0158644 \ldots \\
0.0129923 \ldots \\
0.0100686 \ldots \\
0.0078162 \ldots \\
0.0072943 \ldots
\end{array}\right)
$$


From (7.3) and (7.4), we deduce that

$$
(\mathbf{I}-\mathbf{A})(\mathbf{H}-\mathbf{X}) \geq \mathbf{0} .
$$

Since all elements of $(\mathbf{I}-\mathbf{A})^{-1}$ are positive, it follows that

$$
\mathbf{H} \geq \mathbf{X} .
$$

In particular we have

$$
H(2.2) \geq 0.0223939 .
$$

Now taking $\sigma=\{1\}$ and $s=2.2$ in (3.3) of Lemma 3.2, we find, for $\delta$ sufficiently small, $N_{0}$ sufficiently large and $N \geq N_{0}$,

$$
\begin{aligned}
D(N) & \leq S\left(\mathcal{A} ; \mathcal{P}(N), N^{(1 / 2-\delta) / 2.2}\right)=\Phi(N,\{1\}, 2.2) \\
& \leq\left\{A(2.2)-H_{k, N_{0}}(2.2)\right\} \frac{4 C_{N} \operatorname{li}(N)}{\log \left(N^{1 / 2-\delta}\right)} \\
& \leq 8(1-0.0223938) \Theta(N) \leq 7.82085 \Theta(N) .
\end{aligned}
$$

This completes the proof of Theorem 1.

REMARK 2. (i) The constant $s_{1}=2.2$ comes from the fact that $\Psi_{2}(s)$ attains the maximal value at $s=s_{1}$ (approximately). Since $H(s)$ is decreasing on $[1,10]$, we have $H(2.1) \geq 0.0223939$. In order to obtain a better lower bound (which leads to a smaller constant than 7.82085), we must look for a new weighted inequality (as in Lemmas 4.1 and 4.2 ) such that the corresponding main term $\Psi(2.1)$ has a larger lower bound than 0.015826357 .

(ii) If we divide the interval $[2,3]$ into more than 9 subintervals, we can certainly obtain a better result. But the improvement is minuscule.

8. Proof of Theorem 3. In the case of the twin primes problem, we need to sieve the sequence

$$
\mathcal{B}:=\{p+2: p \leq x\}
$$

Referring to Lemmas 2.7 and 2.9, we have $4 / 7$ for the level of distribution in place $1 / 2$ in the Bombieri-Vinogradov theorem. Thus we can take $Q:=$ $x^{4 / 7-\delta}$ and $\underline{d}:=Q / d$ in the definitions of Section 3. As before, we can prove the corresponding Propositions 3 and 4 with the following modification: In the definition of $\Psi_{1}(s)$ we add a factor $7 / 8$ before $I_{1}(s)$, and in the definition of $\Psi_{2}(s)$ we replace the factor $2 / 5$ before the sum by $7 / 20$. When we use the switching principle to treat the terms $\Omega_{3}$ and $\Gamma_{i}$ for $5 \leq i \leq 21$, the related error terms can be estimated by using Lemma 2.9 which has $4 / 7$ for the level of distribution (see [26, p. 380]). 
Table 2. Choice of parameters

\begin{tabular}{rccccccc}
\hline$i$ & $s_{i}$ & $s_{i}^{\prime}$ & $\kappa_{1, i}$ & $\kappa_{2, i}$ & $\kappa_{3, i}$ & $\Psi_{1}\left(s_{i}\right)$ & $\Psi_{2}\left(s_{i}\right)$ \\
\hline 1 & 2.1 & 4.93 & 3.62 & 2.86 & 2.34 & & 0.020914508 \\
2 & 2.2 & 4.91 & 3.62 & 2.85 & 2.33 & & 0.020399717 \\
3 & 2.3 & 5.00 & 3.63 & 2.82 & 2.30 & & 0.019005124 \\
4 & 2.4 & 4.52 & 3.64 & 2.87 & 2.40 & & 0.016618139 \\
5 & 2.5 & 3.72 & & & & 0.013597508 & \\
6 & 2.6 & 3.62 & & & & 0.010644985 & \\
7 & 2.7 & 3.49 & & & & 0.007155027 & \\
8 & 2.8 & 3.35 & & & & 0.003741586 & \\
9 & 2.9 & 3.19 & & & & 0.001087780 & \\
10 & 3.0 & 3.00 & & & & 0.000000000 & \\
\hline
\end{tabular}

As before we can prove

$$
H(2.1) \geq 0.0287118 \text {. }
$$

Thus for $\delta$ sufficiently small, $x_{0}$ sufficiently large and $x \geq x_{0}$, we have

$$
\begin{aligned}
\pi_{2}(x) & \leq S\left(\mathcal{B} ; \mathcal{P}(2), x^{(1 / 2-\delta) / 2.1}\right) \\
& \leq 3.5(1-0.0287117) \Pi(x) \leq 3.39951 \Pi(x) .
\end{aligned}
$$

This completes the proof of Theorem 3 .

9. Chen's system of weights. Let

$$
\mathcal{A}:=\{N-p: p \leq N\}, \quad \mathcal{P}(q):=\{p:(p, q)=1\} .
$$

The inequality $(9.1)$ below appeared in [9] (page 479, $(11)$ ) with $(\kappa, \sigma)=$ $\left(\frac{1}{12}, \frac{1}{3.047}\right),\left(\frac{1}{9.2}, \frac{1}{3.41}\right)$ without proof. Cai [3] gave a proof with an extra assumption $3 \sigma+\kappa>1$. Here we present a proof without Cai's assumption. This removal is important in our argument.

Lemma 9.1. Let $0<\kappa<\sigma<1 / 3$. Then

$$
D_{1,2}(N) \geq S\left(\mathcal{A} ; \mathcal{P}(N), N^{\kappa}\right)-\frac{1}{2} S_{1}-S_{2}-\frac{1}{2} S_{3}+\frac{1}{2} S_{4}+O\left(N^{1-\kappa}\right),
$$

where $S_{i}=S_{i}(\kappa, \sigma)(1 \leq i \leq 4)$ are defined by

$$
\begin{aligned}
& S_{1}:=\sum_{\substack{N^{\kappa} \leq p<N^{\sigma} \\
(p, N)=1}} S\left(\mathcal{A}_{p} ; \mathcal{P}(N), N^{\kappa}\right), \\
& S_{2}:=\sum_{\substack{N^{\sigma} \leq p_{1}<p_{2}<\left(N / p_{1}\right)^{1 / 2} \\
\left(p_{1} p_{2}, N\right)=1}} S\left(\mathcal{A}_{p_{1} p_{2}} ; \mathcal{P}\left(N p_{1}\right), p_{2}\right), \\
&\left.S^{\prime}\right)
\end{aligned}
$$




$$
\begin{aligned}
S_{3} & :=\sum_{\substack{N^{\kappa} \leq p_{1}<N^{\sigma} \leq p_{2}<\left(N / p_{1}\right)^{1 / 2} \\
\left(p_{1} p_{2}, N\right)=1}} S\left(\mathcal{A}_{p_{1} p_{2}} ; \mathcal{P}\left(N p_{1}\right), p_{2}\right), \\
S_{4} & \left.:=\sum_{\substack{N^{\kappa} \leq p_{1}<p_{2}<p_{3}<N^{\sigma} \\
\left(p_{1} p_{2} p_{3}, N\right)=1}} \sum_{\substack{\mathcal{A}_{p_{1} p_{2} p_{3}} \\
\sum_{\mathcal{1}}}} \mathcal{P}\left(N p_{1}\right), p_{2}\right) .
\end{aligned}
$$

Proof. Clearly the desired inequality (9.1) is equivalent to

$$
\begin{aligned}
D_{1,2}(N) \geq & \sum_{a \in \mathcal{A},\left(a, P\left(N^{\kappa}\right)\right)=1}\left(1-\frac{1}{2} s_{1}(a)-s_{2}(a)-\frac{1}{2} s_{3}(a)+\frac{1}{2} s_{4}(a)\right) \\
& +O\left(N^{1-\kappa}\right)
\end{aligned}
$$

where

$$
\begin{aligned}
& s_{1}(a):=\sum_{\substack{N^{\kappa} \leq p<N^{\sigma} \\
p \mid a,(p, N)=1}} 1, \\
& s_{2}(a):=\sum_{\substack{N^{\sigma} \leq p_{1}<p_{2}<\left(N / p_{1}\right)^{1 / 2} \\
p_{1} p_{2}\left|a,\left(p_{1} p_{2}, N\right)=1 \\
p\right|\left(a / p_{1} p_{2}\right) \Rightarrow p \geq p_{2}}} 1, \\
& s_{3}(a):=\sum_{\substack{N^{\kappa} \leq p_{1}<N^{\sigma} \leq p_{2}<\left(N / p_{1}\right)^{1 / 2} \\
p_{1} p_{2}\left|a,\left(p_{1} p_{2}, N\right)=1 \\
p\right|\left(a / p_{1} p_{2}\right) \Rightarrow p \geq p_{2}}} 1, \\
& s_{4}(a):=\sum_{\substack{N^{\kappa} \leq p_{1}<p_{2}<p_{3}<N^{\sigma} \\
p_{1} p_{2} p_{3} \mid a,\left(p_{1} p_{2} p_{3}, N\right)=1}} \sum_{\substack{p^{\prime} \\
p\left(a / p_{1}\right.}} 1 . \\
& p \mid\left(a / p_{1} p_{2} p_{3}\right) \Rightarrow p \geq p_{2}
\end{aligned}
$$

Let

$$
\delta^{*}(a):= \begin{cases}1 & \text { if } \Omega(a) \leq 2 \\ 0 & \text { otherwise }\end{cases}
$$

Then it is easy to see that

$$
\begin{aligned}
D_{1,2}(N) & \geq \sum_{a \in \mathcal{A},\left(a, P\left(N^{\kappa}\right)\right)=1} \delta^{*}(a) \\
& =\sum_{a \in \mathcal{A},\left(a, P\left(N^{\kappa}\right)\right)=1} \mu(a)^{2} \delta^{*}(a)+O\left(N^{1 / 2}\right),
\end{aligned}
$$

where we have used the fact that

$$
\sum_{\substack{\left.a \in \mathcal{A} \\ a, P\left(N^{\kappa}\right)\right)=1}}\left\{1-\mu(a)^{2}\right\} \delta^{*}(a) \leq \sum_{N^{\kappa} \leq p \leq N^{1 / 2}} 1 \ll N^{1 / 2}
$$


Similarly if we write

$$
\delta(a):=1-\frac{1}{2} s_{1}(a)-s_{2}(a)-\frac{1}{2} s_{3}(a)+\frac{1}{2} s_{4}(a),
$$

we can show that

$$
\sum_{a \in \mathcal{A},\left(a, P\left(N^{\kappa}\right)\right)=1} \delta(a)=\sum_{a \in \mathcal{A},\left(a, P\left(N^{\kappa}\right)\right)=1} \mu(a)^{2} \delta(a)+O\left(N^{1-\kappa}\right) .
$$

Thus in order to prove (9.2) it suffices to verify that

$$
\delta^{*}(a) \geq \delta(a)
$$

for $a \in \mathcal{A}, \mu(a)^{2}=1$ and $\left(a, P\left(N^{\kappa}\right)\right)=(a, N)=1$.

We first observe that (9.3) is trivial if $\Omega(a) \leq 2$, since $\delta^{*}(a)=1$ and $s_{4}(a)=0$ in this case. It remains to show that $\delta(a) \leq 0$ in all other cases, which can be verified as follows:

- If $\Omega(a)=3$ and $s_{1}(a)=0$, then $s_{2}(a)=1$ and $s_{3}(a)=s_{4}(a)=0$. Thus $\delta(a)=0$.

- If $\Omega(a)=3$ and $s_{1}(a)=1$, then $s_{3}(a)=1$ and $s_{2}(a)=s_{4}(a)=0$. Thus $\delta(a)=0$.

- If $\Omega(a)=3$ and $s_{1}(a)=2$, then $s_{2}(a)=s_{3}(a)=s_{4}(a)=0$. Thus $\delta(a)=0$.

- If $\Omega(a)=3$ and $s_{1}(a)=3$, then $s_{2}(a)=s_{3}(a)=0$ and $s_{4}(a)=1$. Thus $\delta(a)=0$.

- If $\Omega(a) \geq 4$ and $s_{1}(a)=1$, then $s_{3}(a)=1$ and $s_{2}(a)=s_{4}(a)=0$. Thus $\delta(a)=0$.

- If $\Omega(a) \geq 4$ and $s_{1}(a)=2$, then $s_{2}(a)=s_{3}(a)=s_{4}(a)=0$. Thus $\delta(a)=0$.

- If $\Omega(a) \geq 4$ and $s_{1}(a) \geq 3$, then $s_{2}(a)=s_{3}(a)=0$ and $s_{4}(a)=$ $s_{1}(a)-2$. Thus $\delta(a)=0$. This completes the proof.

The main difference between (9.1) and Chen's other weighted inequalities (see (34) of [7] and page 425 of [8]) is the additional positive term $S_{4}$. However a direct application of the sieve to $S_{4}$ leads to a zero contribution. In order to take advantage of $S_{4}$, Chen used (9.1) with two different couples of parameters $(\kappa, \sigma)$. Then a compatible application of the Buchstab identity and switching principle leads to some compensation. This idea was also used by Cai \& Lu [4] and Cai [3]. Here we make some modifications of their argument to make this process more powerful.

LEMMA 9.2. Let $0<\kappa_{1}<\kappa_{2}<\varrho<\sigma_{2}<\sigma_{1}<1 / 3$ be such that $3 \kappa_{1}+\varrho \geq 1 / 2$. Then 


$$
\begin{aligned}
4 D_{1,2}(N) \geq & 4 S\left(\mathcal{A} ; \mathcal{P}(N), N^{\kappa_{1}}\right)-\Upsilon_{1}-\Upsilon_{2}-\Upsilon_{3} \\
& +\Upsilon_{4}+\Upsilon_{5}+\Upsilon_{6}-2 \Upsilon_{7}-2 \Upsilon_{8}-\Upsilon_{9}-\Upsilon_{10} \\
& +\Upsilon_{11}+\Upsilon_{12}-\Upsilon_{13}-\Upsilon_{14}+\Upsilon_{15}+O\left(N^{1-\kappa_{1}}\right)
\end{aligned}
$$

where

$$
\begin{aligned}
& \Upsilon_{1}:=\sum_{\substack{N^{\kappa_{1}} \leq p<N^{\kappa_{2}} \\
(p, N)=1}} S\left(\mathcal{A}_{p} ; \mathcal{P}(N), p\right), \\
& \Upsilon_{2}:=\sum_{\substack{N^{\kappa_{1}} \leq p<N^{\sigma_{1}} \\
(p, N)=1}} S\left(\mathcal{A}_{p} ; \mathcal{P}(N), N^{\kappa_{1}}\right), \\
& \Upsilon_{3}:=\sum_{\substack{N^{\kappa_{1}} \leq p<N^{\sigma_{2}} \\
(p, N)=1}} S\left(\mathcal{A}_{p} ; \mathcal{P}(N), N^{\kappa_{1}}\right), \\
& \Upsilon_{4}:=\sum_{\substack{N^{\kappa_{1}} \leq p_{1}<p_{2}<N^{\kappa_{2}} \\
\left(p_{1} p_{2}, N\right)=1}} S\left(\mathcal{A}_{p_{1} p_{2}} ; \mathcal{P}(N), N^{\kappa_{1}}\right), \\
& \Upsilon_{5}:=\sum_{\substack{N^{\kappa_{1}} \leq p_{1}<N^{\kappa_{2}} \leq p_{2}<N^{1 / 2-2 \kappa_{1}} / p_{1} \\
\left(p_{1} p_{2}, N\right)=1}} S\left(\mathcal{A}_{p_{1} p_{2}} ; \mathcal{P}(N), N^{\kappa_{1}}\right),
\end{aligned}
$$

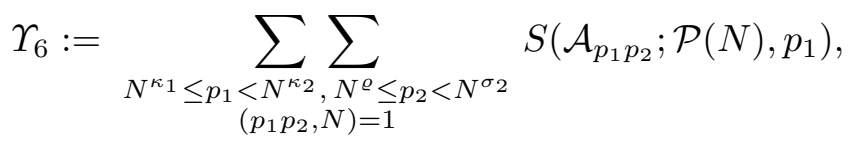

$$
\begin{aligned}
& \Upsilon_{7}:=\sum_{\substack{N^{\sigma_{1}} \leq p_{1}<p_{2}<\left(N / p_{1}\right)^{1 / 2} \\
\left(p_{1} p_{2}, N\right)=1}} S\left(\mathcal{A}_{p_{1} p_{2}} ; \mathcal{P}\left(N p_{1}\right), p_{2}\right), \\
& \Upsilon_{8}:=\sum_{\substack{N^{\sigma_{2}} \leq p_{1}<p_{2}<\left(N / p_{1}\right)^{1 / 2} \\
\left(p_{1} p_{2}, N\right)=1}} S\left(\mathcal{A}_{p_{1} p_{2}} ; \mathcal{P}\left(N p_{1}\right), p_{2}\right), \\
& \Upsilon_{9}:=\sum_{\substack{N^{\kappa_{1}} \leq p_{1}<N^{\sigma_{1}} \leq p_{2}<\left(N / p_{1}\right)^{1 / 2} \\
\left(p_{1} p_{2}, N\right)=1}} S\left(\mathcal{A}_{p_{1} p_{2}} ; \mathcal{P}\left(N p_{1}\right), p_{2}\right), \\
& \Upsilon_{10}:=\sum_{\substack{N^{\kappa_{2}} \leq p_{1}<N^{\sigma_{2}} \leq p_{2}<\left(N / p_{1}\right)^{1 / 2} \\
\left(p_{1} p_{2}, N\right)=1}} S\left(\mathcal{A}_{p_{1} p_{2}} ; \mathcal{P}\left(N p_{1}\right), N^{\sigma_{1}}\right), \\
& \Upsilon_{11}:=\sum_{\substack{N^{\kappa_{2}} \leq p_{1}<p_{2}<p_{3}<N^{\sigma_{2}} \\
\left(p_{1} p_{2} p_{3}, N\right)=1}} S\left(\mathcal{A}_{p_{1} p_{2} p_{3}} ; \mathcal{P}(N), p_{2}\right), \\
& \Upsilon_{12}:=\sum_{\substack{N^{\kappa_{2}} \leq p_{1}<N^{\sigma_{2}}, N^{\sigma_{1}} \leq p_{2}<p_{3}<\left(N / p_{1}\right)^{1 / 2} \\
\left(p_{1} p_{2} p_{3}, N\right)=1}} S\left(\mathcal{A}_{p_{1} p_{2} p_{3}} ; \mathcal{P}\left(N p_{1}\right), p_{2}\right) \text {, }
\end{aligned}
$$




$$
\begin{aligned}
\Upsilon_{13}:=\sum_{\substack{N^{\kappa} 1 \\
\leq p_{1}<p_{2}<p_{3}<p_{4}<N^{\kappa_{2}} \\
\left(p_{1} p_{2} p_{3} p_{4}, N\right)=1}} S\left(\mathcal{A}_{p_{1} p_{2} p_{3} p_{4}} ; \mathcal{P}(N), p_{2}\right), \\
\Upsilon_{14}:=\sum_{\substack{N^{\kappa} 1 \leq p_{1}<p_{2}<p_{3}<N^{\kappa} \kappa_{2} \leq p_{4}<N^{1 / 2-2 \kappa_{1} / p_{3}} \\
\left(p_{1} p_{2} p_{3} p_{4}, N\right)=1}} S\left(\mathcal{A}_{p_{1} p_{2} p_{3} p_{4}} ; \mathcal{P}(N), p_{2}\right), \\
\Upsilon_{15}:=\sum_{\substack{N^{\kappa_{2}} \leq p_{1}<N^{\sigma_{2}} \leq p_{2}<p_{3}<p_{4}<N^{\sigma_{1}} \\
\left(p_{1} p_{2} p_{3} p_{4}, N\right)=1}} S\left(\mathcal{A}_{p_{1} p_{2} p_{3} p_{4}} ; \mathcal{P}(N), p_{3}\right) .
\end{aligned}
$$

Proof. The inequality $(9.1)$ with $(\kappa, \sigma)=\left(\kappa_{2}, \sigma_{2}\right)$ implies

$$
\begin{aligned}
2 D_{1,2}(N) \geq & 2 S\left(\mathcal{A} ; \mathcal{P}(N), N^{\kappa_{2}}\right)-S_{1}\left(\kappa_{2}, \sigma_{2}\right) \\
& -2 \Upsilon_{8}-S_{3}\left(\kappa_{2}, \sigma_{2}\right)+\Upsilon_{11}+O\left(N^{1-\kappa_{2}}\right) .
\end{aligned}
$$

Buchstab's identity, when applied three times, gives the equality

$$
\begin{aligned}
2 S\left(\mathcal{A} ; \mathcal{P}(N), N^{\kappa_{2}}\right)= & 2 S\left(\mathcal{A} ; \mathcal{P}(N), N^{\kappa_{1}}\right)-\Upsilon_{1} \\
& -\sum_{\substack{N^{\kappa} 1 \leq p<N^{\kappa_{2}} \\
(p, N)=1}} S\left(\mathcal{A}_{p} ; \mathcal{P}(N), N^{\kappa_{1}}\right) \\
& +\Upsilon_{4}-\sum_{\substack{N^{\kappa} 1 \leq p_{1}<p_{2}<p_{3}<N^{\kappa_{2}} \\
\left(p_{1} p_{2} p_{3}, N\right)=1}} \sum_{\left(\mathcal{A}_{p_{1} p_{2} p_{3}} ; \mathcal{P}(N), p_{1}\right) .} \sum .
\end{aligned}
$$

Similarly a simple application of Buchstab's identity yields

$$
\begin{aligned}
& S_{1}\left(\kappa_{2}, \sigma_{2}\right)=\sum_{\substack{N^{\kappa_{2}} \leq p<N^{\sigma_{2}} \\
(p, N)=1}} S\left(\mathcal{A}_{p} ; \mathcal{P}(N), N^{\kappa_{1}}\right) \\
& -\sum_{\substack{N^{\kappa_{1}} \leq p_{1}<N^{\kappa_{2}} \leq p_{2}<N^{\varrho} \\
\left(p_{1} p_{2}, N\right)=1}} S\left(\mathcal{A}_{p_{1} p_{2}} ; \mathcal{P}(N), p_{1}\right)-\Upsilon_{6} .
\end{aligned}
$$

Clearly $p_{1}<N^{\sigma_{2}}$ and $\sigma_{2}<\sigma_{1}<1 / 3$ imply that $N^{\sigma_{1}}<\left(N / p_{1}\right)^{1 / 2}$. Thus by Buchstab's identity, we can write

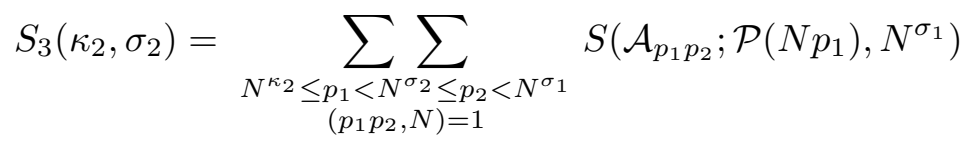

$$
\begin{aligned}
& +\sum_{\substack{N^{\kappa_{2}} \leq p_{1}<N^{\sigma_{2}} \leq p_{2}<p_{3}<N^{\sigma_{1}} \\
\left(p_{1} p_{2} p_{3}, N\right)=1}} S\left(\mathcal{A}_{p_{1} p_{2} p_{3}} ; \mathcal{P}\left(N p_{1}\right), p_{3}\right) \\
& +O\left(N^{1-\sigma_{2}}\right)
\end{aligned}
$$




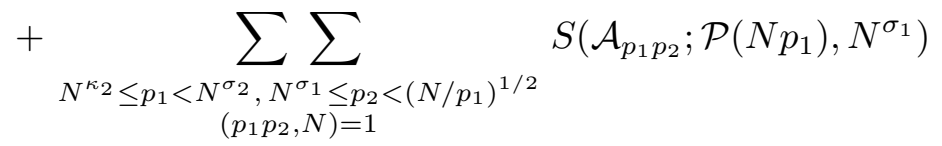

$$
\begin{aligned}
& -\sum_{\substack{N^{\kappa_{2}} \leq p_{1}<N^{\sigma_{2}}, N^{\sigma_{1}} \leq p_{2}<p_{3}<\left(N / p_{1}\right)^{1 / 2} \\
\left(p_{1} p_{2} p_{3}, N\right)=1}} S\left(\mathcal{A}_{p_{1} p_{2} p_{3}} ; \mathcal{P}\left(N p_{1}\right), p_{2}\right) \\
& =\Upsilon_{10}-\Upsilon_{12} \\
& +\sum_{\substack{N^{\kappa_{2}} \leq p_{1}<N^{\sigma_{2}} \leq p_{2}<p_{3}<N^{\sigma_{1}} \\
\left(p_{1} p_{2} p_{3}, N\right)=1}} S\left(\mathcal{A}_{p_{1} p_{2} p_{3}} ; \mathcal{P}\left(N p_{1}\right), p_{3}\right)+O\left(N^{1-\sigma_{2}}\right) .
\end{aligned}
$$

Inserting these into (9.5), we find that

$$
\begin{aligned}
2 D_{1,2}(N) \geq & 2 S\left(\mathcal{A} ; \mathcal{P}(N), N^{\kappa_{1}}\right)-\Upsilon_{1}-\Upsilon_{3}+\Upsilon_{4}+\Upsilon_{6} \\
& -2 \Upsilon_{8}-\Upsilon_{10}+\Upsilon_{11}+\Upsilon_{12}+\Delta+O\left(N^{1-\kappa_{2}}\right)
\end{aligned}
$$

where

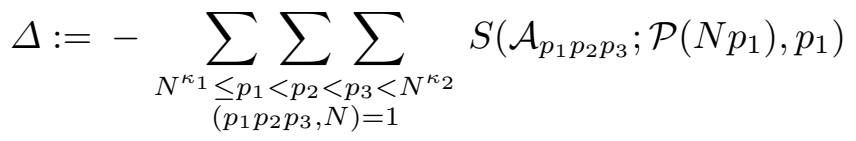

$$
\begin{aligned}
& -\sum_{N^{\kappa_{2}} \leq p_{1}<N^{\sigma_{2}} \leq p_{2}<p_{3}<N^{\sigma_{1}}} \sum_{\substack{\left.p_{1} p_{2} p_{3}, N\right)=1 \\
p_{2}}} S\left(\mathcal{A}_{p_{1} p_{2} p_{3}} ; \mathcal{P}\left(N p_{1}\right), p_{3}\right)
\end{aligned}
$$

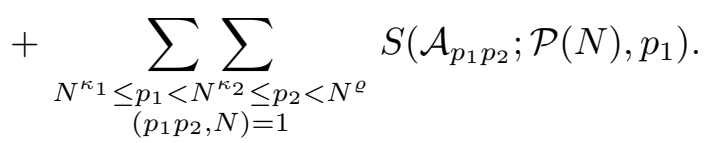

Next we shall further decompose $\Delta$. In view of $3 \kappa_{1}+\varrho \geq 1 / 2$, we have $N^{\varrho} \geq N^{1 / 2-2 \kappa_{1}} / p_{1}$ provided $p_{1} \geq N^{\kappa_{1}}$. Thus Buchstab's identity allows us to write

$$
\begin{aligned}
& \sum_{\substack{N^{\kappa} 1 \\
N_{1}<N^{\kappa_{2}} \leq p_{2}<N^{\varrho} \\
\left(p_{1} p_{2}, N\right)=1}} S\left(\mathcal{A}_{p_{1} p_{2}} ; \mathcal{P}(N), p_{1}\right) \\
& \geq \Upsilon_{5}-\sum_{\substack{N^{\kappa} 1 \leq p_{1}<p_{2}<N^{\kappa_{2}} \leq p_{3}<N^{1 / 2-2 \kappa_{1} / p_{2}} \\
\left(p_{1} p_{2} p_{3}, N\right)=1}} S\left(\mathcal{A}_{p_{1} p_{2} p_{3}} ; \mathcal{P}(N), p_{1}\right) .
\end{aligned}
$$

Thus we have

$$
\Delta \geq \Upsilon_{5}+\Delta_{1}
$$

where

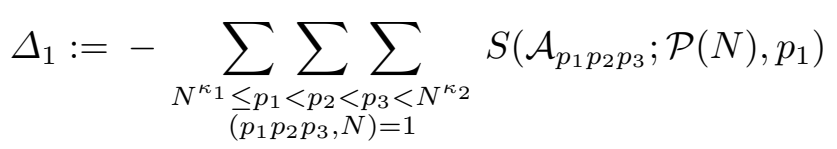




$$
\begin{aligned}
& -\sum_{\substack{N^{\kappa_{1}} \leq p_{1}<p_{2}<N^{\kappa_{2}} \leq p_{3}<N^{1 / 2-2 \kappa_{1}} / p_{2} \\
\left(p_{1} p_{2} p_{3}, N\right)=1}} S\left(\mathcal{A}_{p_{1} p_{2} p_{3}} ; \mathcal{P}(N), p_{1}\right) \\
& -\sum_{\substack{N^{\kappa_{2}} \leq p_{1}<N^{\sigma_{2}} \leq p_{2}<p_{3}<N^{\sigma_{1}} \\
\left(p_{1} p_{2} p_{3}, N\right)=1}} S\left(\mathcal{A}_{p_{1} p_{2} p_{3}} ; \mathcal{P}\left(N p_{1}\right), p_{3}\right) .
\end{aligned}
$$

Now the inequality (9.6) becomes

$$
\begin{aligned}
2 D_{1,2}(N) \geq & 2 S\left(\mathcal{A} ; \mathcal{P}(N), N^{\kappa_{1}}\right)-\Upsilon_{1}-\Upsilon_{3}+\Upsilon_{4}+\Upsilon_{5}+\Upsilon_{6} \\
& -2 \Upsilon_{8}-\Upsilon_{10}+\Upsilon_{11}+\Upsilon_{12}+\Delta_{1}+O\left(N^{1-\kappa_{2}}\right) .
\end{aligned}
$$

The inequality $(9.1)$ with $(\kappa, \sigma)=\left(\kappa_{1}, \sigma_{1}\right)$ gives

$$
\begin{aligned}
2 D_{1,2}(N) \geq & 2 S\left(\mathcal{A} ; \mathcal{P}(N), N^{\kappa_{1}}\right)-\Upsilon_{2}-2 \Upsilon_{7} \\
& -\Upsilon_{9}+S_{4}\left(\kappa_{1}, \sigma_{1}\right)+O\left(N^{1-\kappa_{1}}\right) .
\end{aligned}
$$

Adding (9.7) to (9.8) yields

$$
\begin{aligned}
4 D_{1,2}(N) \geq & 4 S\left(\mathcal{A} ; \mathcal{P}(N), N^{\kappa_{1}}\right)-\Upsilon_{1}-\Upsilon_{2}-\Upsilon_{3} \\
& +\Upsilon_{4}+\Upsilon_{5}+\Upsilon_{6}-2 \Upsilon_{7}-2 \Upsilon_{8}-\Upsilon_{9} \\
& -\Upsilon_{10}+\Upsilon_{11}+\Upsilon_{12}+\Delta_{2}+O\left(N^{1-\kappa_{1}}\right)
\end{aligned}
$$

where

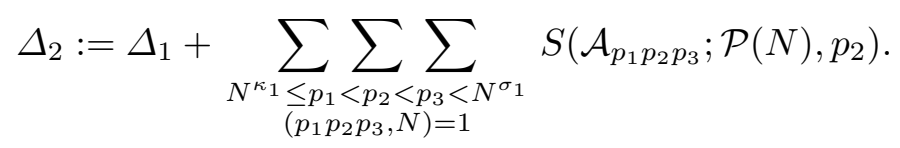

Clearly the summation domains in the three terms on the right-hand side of $\Delta_{1}$ are distinct and are contained in the summation domain of the last triple sums on the right-hand side of $\Delta_{2}\left(\right.$ since $\left.3 \kappa_{1}+\sigma_{1}>3 \kappa_{1}+\varrho \geq 1 / 2\right)$. Therefore

$$
\begin{aligned}
& \Delta_{2} \geq-\sum_{N^{\kappa_{1}} \leq p_{1}<p_{2}<p_{3}<N^{\kappa_{2}}}\left\{S\left(\mathcal{A}_{p_{1} p_{2} p_{3}} ; \mathcal{P}(N), p_{1}\right)-S\left(\mathcal{A}_{p_{1} p_{2} p_{3}} ; \mathcal{P}(N), p_{2}\right)\right\} \\
& -\sum_{N^{\kappa_{1}} \leq p_{1}<p_{2}<N^{\kappa_{2}} \leq p_{3}<N^{1 / 2-2 \kappa_{1}} / p_{2}}\left\{\sum _ { ( p _ { 1 } p _ { 2 } p _ { 3 } , N ) = 1 } \left\{S\left(\mathcal{A}_{p_{1} p_{2} p_{3}} ; \mathcal{P}(N), p_{1}\right)\right.\right. \\
& \left.-S\left(\mathcal{A}_{p_{1} p_{2} p_{3}} ; \mathcal{P}(N), p_{2}\right)\right\} \\
& +\sum_{\substack{N^{\kappa_{2}} \leq p_{1}<N^{\sigma_{2}} \leq p_{2}<p_{3}<N^{\sigma_{1}} \\
\left(p_{1} p_{2} p_{3}, N\right)=1}}\left\{S\left(\mathcal{A}_{p_{1} p_{2} p_{3}} ; \mathcal{P}(N), p_{2}\right)\right. \\
& \left.-S\left(\mathcal{A}_{p_{1} p_{2} p_{3}} ; \mathcal{P}(N), p_{3}\right)\right\} \\
& =-\Upsilon_{13}-\Upsilon_{14}+\Upsilon_{15}+O\left(N^{1-\kappa_{1}}\right) .
\end{aligned}
$$

Combining this with (9.9), we obtain the required result. This completes the proof. 
REMARK 3. Lemmas 9.1 and 9.2 are also valid for

$$
\begin{aligned}
\mathcal{A}^{\prime} & :=\{p+2: p \leq x\}, \\
\mathcal{A}^{\prime \prime} & :=\left\{p+2: x<p \leq x+x^{\theta}\right\}, \\
\mathcal{A}^{\prime \prime \prime} & :=\left\{N-p: \alpha N<p \leq \alpha N+N^{\theta}\right\},
\end{aligned}
$$

if we make some suitable modifications. For example, we have

$$
\begin{aligned}
4 \pi_{1,2}(x) \geq & 4 S\left(\mathcal{A}^{\prime} ; \mathcal{P}(2), x^{\kappa_{1}}\right)-\Upsilon_{1}^{\prime}-\Upsilon_{2}^{\prime}-\Upsilon_{3}^{\prime} \\
& +\Upsilon_{4}^{\prime}+\Upsilon_{5}^{\prime}+\Upsilon_{6}^{\prime}-2 \Upsilon_{7}^{\prime}-2 \Upsilon_{8}^{\prime}-\Upsilon_{9}^{\prime}-\Upsilon_{10}^{\prime} \\
& +\Upsilon_{11}^{\prime}+\Upsilon_{12}^{\prime}-\Upsilon_{13}^{\prime}-\Upsilon_{14}^{\prime}+\Upsilon_{15}^{\prime}+O\left(N^{1-\kappa_{1}}\right),
\end{aligned}
$$

where $\Upsilon_{j}^{\prime}$ is defined similarly to $\Upsilon_{j}$ with $\mathcal{A}$ replaced by $\mathcal{A}^{\prime}, \mathcal{P}(N)$ by $\mathcal{P}(2)$, $\mathcal{P}\left(N p_{1}\right)$ by $\mathcal{P}\left(2 p_{1}\right),\left(N / p_{1}\right)^{1 / 2}$ by $\left(x / p_{1}\right)^{1 / 2}, N^{1 / 2-2 \kappa_{1}} / p_{2}$ by $x^{4 / 7-2 \kappa_{1}} / p_{2}$ (in $\Upsilon_{5}$ and $\Upsilon_{14}$ ), $N^{\varrho}$ by $x^{\varrho}, N^{\kappa_{i}}$ by $x^{\kappa_{i}}, N^{\sigma_{i}}$ by $x^{\sigma_{i}}$ and with the conditions $(p, N)=1,\left(p_{1} p_{2}, N\right)=1,\left(p_{1} p_{2} p_{3}, N\right)=1$ and $\left(p_{1} p_{2} p_{3} p_{4}, N\right)=1$ omitted. The assumption on the parameters is

$$
0<\kappa_{1}<\kappa_{2}<\varrho<\sigma_{2}<\sigma_{1}<1 / 3, \quad 3 \kappa_{1}+\varrho \geq 4 / 7 .
$$

The last condition is necessary in the proof of $\Delta \geq \Upsilon_{5}^{\prime}+\Delta_{1}$.

10. Proofs of Theorems 2 and 5. For simplicity, we write $\mathcal{L}:=\log N$ and use $B$ to denote a suitable positive constant determined by Lemma 2.3. We shall estimate all terms $\Upsilon_{i}$ in the inequality (9.4). For this we suppose that

$$
\left\{\begin{array}{l}
1 / 12=\kappa_{1}<\kappa_{2} \leq 1 / 8, \quad 1 / 4=\varrho<\sigma_{2}<\sigma_{1}<1 / 3 \\
3 \sigma_{1}+\kappa_{1}>1, \quad 2 \sigma_{1}+\sigma_{2}+\kappa_{2}>1 .
\end{array}\right.
$$

$1^{\circ}$ Lower bound of $S\left(\mathcal{A} ; \mathcal{P}(N), N^{\kappa_{1}}\right)$. We apply (2.5) of Lemma 2.2 with

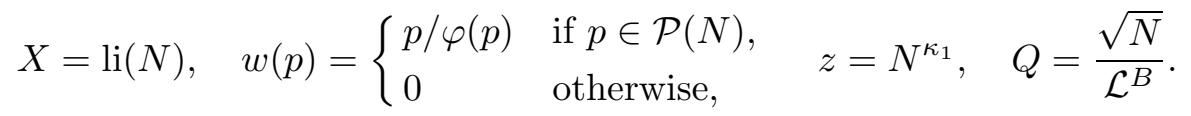

Since $\left|\lambda_{l}^{ \pm}(q)\right| \leq 1$, Lemma 2.3 with the choice $f(1)=1$ and $f(m)=0$ if $m \geq 2$ implies that

$$
\begin{aligned}
\left|\sum_{l<L} \sum_{q \mid P(z)} \lambda_{l}^{ \pm}(q) r(\mathcal{A}, q)\right| & \ll \sum_{\substack{q \leq \sqrt{N} / \mathcal{L}^{B}\\
}} \mu(q)^{2} \max _{y \leq N} \max _{(a, q)=1}\left|\pi(y ; q, a)-\frac{\operatorname{li}(y)}{\varphi(q)}\right| \\
& \ll N / \mathcal{L}^{3} .
\end{aligned}
$$

In view of $V(z) \sim 2 e^{-\gamma} C_{N} / \log z\left(\gamma\right.$ is the Euler constant) and $C_{N} \gg 1$, we deduce that

$$
S\left(\mathcal{A} ; \mathcal{P}(N), N^{\kappa_{1}}\right) \geq\left\{F_{0}+O(\varepsilon)\right\} \Theta(N)
$$


with

$$
F_{0}:=\frac{2 f\left(1 / 2 \kappa_{1}\right)}{\kappa_{1} e^{\gamma}}
$$

$2^{\circ}$ Upper bounds of $\Upsilon_{1}, \Upsilon_{2}$ and $\Upsilon_{3}$. We apply (2.4) of Lemma 2.2 with

$$
X=\frac{\operatorname{li}(N)}{\varphi(p)}, \quad w(p)= \begin{cases}p / \varphi(p) & \text { if } p \in \mathcal{P}(N), \quad z=p, \quad Q=\frac{\sqrt{N}}{p^{B}} \\ 0 & \text { otherwise, }\end{cases}
$$

to $S\left(\mathcal{A}_{p} ; \mathcal{P}(N), p\right)$. The contribution of the error term in $(2.4)$ is

$$
\begin{aligned}
& \ll_{\varepsilon} \sum_{N^{\kappa_{1}} \leq p<N^{\kappa_{2}},(p, N)=1} \sum_{\substack{q \leq \sqrt{N} / p \mathcal{L}^{B}, q \mid P(p) \\
\ll_{\varepsilon}}}|r(\mathcal{A}, p q)| \\
& \sum_{d \leq \sqrt{N} / \mathcal{L}^{B}} \mu(d)^{2} \max _{y \leq N} \max _{(a, d)=1}\left|\pi(y ; d, a)-\frac{\operatorname{li}(y)}{\varphi(d)}\right| \ll_{\varepsilon} \frac{N}{\mathcal{L}^{3}}
\end{aligned}
$$

by Lemma 2.3 with the same choice of $f$ as above. Thus

$$
\Upsilon_{1} \leq \frac{\{1+O(\varepsilon)\} N}{\mathcal{L}} \sum_{N^{\kappa_{1}} \leq p<N^{\kappa_{2}}} \frac{V(p)}{\varphi(p)} F\left(\frac{\log (\sqrt{N} / p)}{\log p}\right)+O\left(\frac{N}{\mathcal{L}^{3}}\right) .
$$

The standard procedure for replacing sums over primes by integrals yields

$$
\Upsilon_{1} \leq\left\{F_{1}+O(\varepsilon)\right\} \Theta(N)
$$

where

$$
F_{1}:=\frac{2}{e^{\gamma}} \int_{\kappa_{1}}^{\kappa_{2}} \frac{F(1 / 2 t-1)}{t^{2}} d t=\frac{4}{e^{\gamma}} \int_{1 / 2 \kappa_{2}-1}^{1 / 2 \kappa_{1}-1} F(t) d t
$$

Similarly we can prove

$$
\Upsilon_{i} \leq\left\{F_{i}+O(\varepsilon)\right\} \Theta(N) \quad(i=2,3),
$$

where

$$
F_{2}:=\frac{4}{e^{\gamma}} \int_{\left(1 / 2-\sigma_{1}\right) / \kappa_{1}}^{\left(1 / 2-\kappa_{1}\right) / \kappa_{1}} \frac{F(t)}{1-2 \kappa_{1} t} d t, \quad F_{3}:=\frac{4}{e^{\gamma}} \int_{\left(1 / 2-\sigma_{2}\right) / \kappa_{1}}^{\left(1 / 2-\kappa_{1}\right) / \kappa_{1}} \frac{F(t)}{1-2 \kappa_{1} t} d t
$$

$3^{\circ}$ Lower bounds of $\Upsilon_{4}$ and $\Upsilon_{5}$. As before we can deduce from Lemmas 2.2 and 2.3 that

$$
\Upsilon_{i} \geq\left\{F_{i}+O(\varepsilon)\right\} \Theta(N) \quad(i=4,5)
$$

where

$$
\begin{aligned}
& F_{4}:=\frac{2}{\kappa_{1} e^{\gamma}} \int_{\kappa_{1}}^{\kappa_{2}} \frac{d t}{t} \int_{t}^{\kappa_{2}} f\left(\frac{1 / 2-t-u}{\kappa_{1}}\right) \frac{d u}{u} \\
& F_{5}:=\frac{2}{\kappa_{1} e^{\gamma}} \int_{\kappa_{1}}^{\kappa_{2}} \frac{d t}{t} \int_{\kappa_{2}}^{1 / 2-2 \kappa_{1}-t} f\left(\frac{1 / 2-t-u}{\kappa_{1}}\right) \frac{d u}{u} .
\end{aligned}
$$


We have used the following fact to remove the condition $\left(p_{1} p_{2}, N\right)=1$ :

$$
\sum_{\substack{p_{1} \mid N \\ p_{1} \geq N^{\kappa}}} \sum_{N^{\kappa} \leq p_{2}<N^{\sigma}} \frac{N}{p_{1} p_{2}}+\sum_{N^{\kappa} \leq p_{1}<N^{\sigma}} \sum_{\substack{p_{2} \mid N \\ p_{2} \geq N^{\kappa}}} \frac{N}{p_{1} p_{2}} \ll N^{1-\kappa} \mathcal{L} .
$$

$4^{\circ}$ Upper bounds of $\Upsilon_{i}$ for $i=7,8,9,10,13,14$. We shall only majorize $\Upsilon_{7}$ and the others can be treated similarly.

Since $\sigma_{1}>1 / 4$, the quantity $\Upsilon_{7}$ is equal to the number of primes $p \leq N$ such that $N-p=p_{1} p_{2} p_{3}$ with $N^{\sigma_{1}} \leq p_{1}<p_{2}<\left(N / p_{1}\right)^{1 / 2}, p_{3} \geq p_{2}$ and $\left(p_{1} p_{2} p_{3}, N\right)=1$. Define

$$
\begin{aligned}
\mathcal{M} & :=\left\{m: m=p_{1} p_{2}, N^{\sigma_{1}} \leq p_{1}<p_{2}<\left(N / p_{1}\right)^{1 / 2},\left(p_{1} p_{2}, N\right)=1\right\}, \\
\mathcal{B} & :=\{b: b=N-m p \leq N, m \in \mathcal{M}, p \leq N / m\} .
\end{aligned}
$$

It is clear that

$$
\Upsilon_{7} \leq S\left(\mathcal{B} ; \mathcal{P}(N), N^{1 / 2}\right)+O\left(N^{1 / 2}\right) .
$$

By applying (2.4) of Lemma 2.2 with

$$
X=\sum_{m \in \mathcal{M}} \operatorname{li}\left(\frac{N}{m}\right), \quad w(p)=\left\{\begin{array}{ll}
p / \varphi(p) & \text { if } p \in \mathcal{P}(N), \\
0 & \text { otherwise, }
\end{array} \quad Q=\frac{\sqrt{N}}{\mathcal{L}^{B}},\right.
$$

we obtain

$$
\Upsilon_{7} \leq \frac{8 C_{N} X}{\log N}\{1+O(\varepsilon)\}+O_{\varepsilon}\left(\sqrt{N}+R_{3}+R_{4}\right)
$$

where

$$
\begin{aligned}
& R_{3}:=\sum_{\substack{q \leq \sqrt{N} / \mathcal{L}^{B} \\
(q, N)=1}} \mu(q)^{2}\left|\sum_{\substack{m \in \mathcal{M} \\
(q, m)=1}}\left(\sum_{\substack{m p \leq N \\
m p \equiv N(\bmod q)}} 1-\frac{\operatorname{li}(N / m)}{\varphi(q)}\right)\right|, \\
& R_{4}:=\sum_{q \leq \sqrt{N} / \mathcal{L}^{B},(q, N)=1} \frac{\mu(q)^{2}}{\varphi(q)} \sum_{m \in \mathcal{M},(q, m)>1} \operatorname{li}\left(\frac{N}{m}\right) .
\end{aligned}
$$

Let $f(m)$ be the characteristic function of $\mathcal{M}$. Since $m \leq N^{3 / 4}$ for $m \in \mathcal{M}$, Lemma 2.3 implies

$$
\begin{aligned}
(10.7) \quad R_{3} & =\sum_{\substack{q \leq \sqrt{N} / \mathcal{L}^{B} \\
(q, N)=1}} \mu(q)^{2}\left|\sum_{\substack{m \leq N^{5 / 6} \\
(q, m)=1}} f(m)\left(\sum_{\substack{m p \leq N \\
m p \equiv N(\bmod q)}} 1-\frac{\operatorname{li}(N / m)}{\varphi(q)}\right)\right| \\
& \ll N / \mathcal{L}^{3} .
\end{aligned}
$$

Noticing that $(d, m)>1$ implies $(d, m) \geq N^{\sigma_{1}}$ for $m \in \mathcal{M}$, we have

$$
R_{4} \ll \frac{N}{\mathcal{L}} \sum_{q \leq \sqrt{N}} \frac{\mu(q)^{2}}{\varphi(q)} \sum_{m \leq N^{3 / 4},(q, m) \geq N^{\sigma_{1}}} \frac{1}{m}
$$




$$
\begin{aligned}
& \ll \frac{N}{\mathcal{L}} \sum_{q \leq N} \frac{\mu(q)^{2}}{\varphi(q)} \sum_{d \mid q, d \geq N^{\sigma_{1}}} \frac{1}{d} \sum_{n \leq N^{3 / 4} / d} \frac{1}{n} \\
& \ll N \sum_{q \leq \sqrt{N}} \frac{\mu(q)^{2}}{\varphi(q)} \sum_{d \mid q, d \geq N^{\sigma_{1}}} \frac{1}{d} \\
& \ll N \sum_{N^{\sigma_{1}<d \leq N}} \frac{1}{d} \sum_{l \leq \sqrt{N} / d} \frac{\mu(d l)^{2}}{\varphi(d l)} \\
& \ll N \sum_{N^{\sigma_{1}<d \leq N}} \frac{\mu(d)^{2}}{d \varphi(d)} \sum_{l \leq \sqrt{N} / d} \frac{\mu(l)^{2}}{\varphi(l)} .
\end{aligned}
$$

Since the function $\mu(n)^{2} / \varphi(n)$ is multiplicative and

$$
\mu\left(p^{\nu}\right)^{2} / \varphi\left(p^{\nu}\right)= \begin{cases}1 /(p-1) & \text { for } \nu=1, \\ 0 & \text { for } \nu \geq 2,\end{cases}
$$

it is plain to see that

$$
\sum_{l \leq t} \frac{\mu(n)^{2}}{\varphi(n)} \asymp \log t .
$$

Thus

$$
R_{4} \ll N^{1-\sigma_{1}} \mathcal{L}^{2} .
$$

By the prime number theorem, we obtain

$$
\begin{aligned}
X & =\{1+o(1)\} \sum_{N^{\sigma_{1}} \leq p_{1}<p_{2}<\left(N / p_{1}\right)^{1 / 2}} \frac{N}{p_{1} p_{2} \log \left(N / p_{1} p_{2}\right)} \\
& =\{1+o(1)\} \frac{N}{\mathcal{L}} \int_{\sigma_{1}}^{1 / 3} \frac{\log (1 / t-2)}{t(1-t)} d t \\
& =\{1+o(1)\} \frac{N}{\mathcal{L}} \int_{2}^{1 / \sigma_{1}-1} \frac{\log (t-1)}{t} d t .
\end{aligned}
$$

Inserting (10.7)-(10.9) into (10.6) yields

$$
\Upsilon_{7} \leq\left\{F_{7}+O(\varepsilon)\right\} \Theta(N),
$$

where

$$
F_{7}:=8 \int_{2}^{1 / \sigma_{1}-1} \frac{\log (t-1)}{t} d t
$$

Similarly we can prove that

$$
\Upsilon_{i} \leq\left\{F_{i}+O(\varepsilon)\right\} \Theta(N) \quad(i=8,10),
$$


where

$$
F_{8}:=8 \int_{2}^{1 / \sigma_{2}-1} \frac{\log (t-1)}{t} d t, \quad F_{10}:=8 \int_{\kappa_{2}}^{\sigma_{2}} \frac{\log \left(1 / \sigma_{2}-1-t / \sigma_{2}\right)}{t(1-t)} d t .
$$

[We need to use the assumption $2 \sigma_{1}+\sigma_{2}+\kappa_{2}>1$ in $\Upsilon_{10}$.]

For the terms $\Upsilon_{9}, \Upsilon_{13}$ and $\Upsilon_{14}$ with $p_{1} \leq N^{1 / 10}$, we can apply Lemma 2.6 instead of Lemma 2.3. A similar argument allows us to show that

$$
\Upsilon_{i} \leq\left\{F_{i}+O(\varepsilon)\right\} \Theta(N) \quad(i=9,13,14)
$$

where

$$
\begin{aligned}
& F_{9}:=\frac{36}{5} \int_{\kappa_{1}}^{1 / 10} \frac{\log \left(1 / \sigma_{1}-1-t / \sigma_{1}\right)}{t(1-t)^{2}} d t \\
& +8 \int_{1 / 10}^{\sigma_{1}} \frac{\log \left(1 / \sigma_{1}-1-t / \sigma_{1}\right)}{t(1-t)} d t \\
& F_{13}:=\frac{36}{5} \int_{\kappa_{1}}^{1 / 10} \frac{d t_{1}}{t_{1}\left(1-t_{1}\right)} \int_{t_{1}}^{\kappa_{2}} \frac{d t_{2}}{t_{2}^{2}} \\
& \times \int_{t_{2}}^{\kappa_{2}} \frac{d t_{3}}{t_{3}} \int_{t_{3}}^{\kappa_{2}} \omega\left(\frac{1-t_{1}-t_{2}-t_{3}-t_{4}}{t_{2}}\right) \frac{d t_{4}}{t_{4}} \\
& +8 \int_{1 / 10}^{\kappa_{2}} \frac{d t_{1}}{t_{1}} \int_{t_{1}}^{\kappa_{2}} \frac{d t_{2}}{t_{2}^{2}} \int_{t_{2}}^{\kappa_{2}} \frac{d t_{3}}{t_{3}} \\
& \times \int_{t_{3}}^{\kappa_{2}} \omega\left(\frac{1-t_{1}-t_{2}-t_{3}-t_{4}}{t_{2}}\right) \frac{d t_{4}}{t_{4}}, \\
& F_{14}:=\frac{36}{5} \int_{\kappa_{1}}^{1 / 10} \frac{d t_{1}}{t_{1}\left(1-t_{1}\right)} \int_{t_{1}}^{\kappa_{2}} \frac{d t_{2}}{t_{2}^{2}} \int_{t_{2}}^{\kappa_{2}} \frac{d t_{3}}{t_{3}} \\
& \times \int_{\kappa_{2}}^{1 / 2-2 \kappa_{1}-t_{3}} \omega\left(\frac{1-t_{1}-t_{2}-t_{3}-t_{4}}{t_{2}}\right) \frac{d t_{4}}{t_{4}} \\
& +8 \int_{1 / 10}^{\kappa_{2}} \frac{d t_{1}}{t_{1}} \int_{t_{1}}^{\kappa_{2}} \frac{d t_{2}}{t_{2}^{2}} \int_{t_{2}}^{\kappa_{2}} \frac{d t_{3}}{t_{3}} \\
& \times \int_{\kappa_{2}}^{1 / 2-2 \kappa_{1}-t_{3}} \omega\left(\frac{1-t_{1}-t_{2}-t_{3}-t_{4}}{t_{2}}\right) \frac{d t_{4}}{t_{4}} .
\end{aligned}
$$

[We need to use the assumption $3 \sigma_{1}+\kappa_{1}>1$ in $\Upsilon_{9}$ and Lemma 2.10 in $\Upsilon_{13}$ and $\left.\Upsilon_{14} \cdot\right]$ 
By inserting (10.2)-(10.5), (10.10)-(10.12) and by using the trivial lower bounds $\Upsilon_{i} \geq 0(i=6,11,12,15)$ into $(9.4)$, we get the inequality

$$
D_{1,2}(N) \geq\left\{F\left(\kappa_{1}, \kappa_{2}, \varrho, \sigma_{2}, \sigma_{1}\right)+O(\varepsilon)\right\} \Theta(N),
$$

where

$$
\begin{aligned}
F\left(\kappa_{1}, \kappa_{2}, \varrho, \sigma_{2}, \sigma_{1}\right):= & \frac{1}{4}\left(4 F_{0}-F_{1}-F_{2}-F_{3}+F_{4}+F_{5}\right. \\
& \left.-2 F_{7}-2 F_{8}-F_{9}-F_{10}-F_{13}-F_{14}\right) .
\end{aligned}
$$

Taking

$$
\kappa_{1}=\frac{1}{12}, \quad \kappa_{2}=\frac{29}{250}, \quad \varrho=\frac{1}{4}, \quad \sigma_{2}=\frac{141}{500}, \quad \sigma_{1}=\frac{41}{125},
$$

a numerical computation gives us

$$
\begin{aligned}
F\left(\kappa_{1}, \kappa_{2}, \varrho, \sigma_{2}, \sigma_{1}\right) \geq & \frac{1}{4}(4 \cdot 13.473613-3.891854-20.432098 \\
& -17.327241+0.697375+2.118119 \\
& -2 \cdot 0.004609-2 \cdot 0.434368 \\
& -5.161945-5.468377-0.023310-0.182860) \\
> & 0.83607 .
\end{aligned}
$$

[For the integrals $F_{13}$ and $F_{14}$, we make use of $\omega(u) \leq 0.561522$ for $u \geq 3.5$.] This completes the proof of Theorem 2 .

Theorem 5 can be proved in the same way. The only difference is to replace Lemmas 2.3 and 2.6 by Lemma 2.4. Here, the choice of parameters is

$$
\begin{aligned}
& \left(\theta, \kappa_{1}, \kappa_{2}, \varrho, \sigma_{2}, \sigma_{1}\right) \\
& \quad=(0.971,(2 \theta-1) / 12,0.111,(2 \theta-1) / 4,0.271,0.313)
\end{aligned}
$$

11. Proof of Theorem 4. The proof of Theorem 4 is very similar to that of Theorem 2. But we must use Lemmas 2.5, 2.7 and 2.8 in place of Lemmas 2.3 and 2.6. In order to take advantage of these lemmas, we must carry out a more careful and delicate analysis. Thus the proof will be slightly complicated.

Suppose that the parameters satisfy the following conditions:

$$
\left\{\begin{array}{l}
2 / 21=\kappa_{1}<\kappa_{2} \leq 1 / 7, \quad 2 / 7=\varrho<\sigma_{2}<29 / 100<\sigma_{1}<1 / 3, \\
3 \sigma_{1}+\kappa_{1}>1, \quad 2 \sigma_{1}+\sigma_{2}+\kappa_{2}>1 .
\end{array}\right.
$$

$1^{\circ}$ Lower bounds of $S\left(\mathcal{A}^{\prime} ; \mathcal{P}(2), x^{\kappa_{1}}\right)$. By $(2.5)$ of Lemma 2.2 and Lemma 2.7 , we can easily prove that

$$
S\left(\mathcal{A}^{\prime} ; \mathcal{P}(2), x^{\kappa_{1}}\right) \geq\left\{G_{0}+O(\varepsilon)\right\} \Pi(x)
$$

with

$$
G_{0}:=\frac{f\left(4 / 7 \kappa_{1}\right)}{\kappa_{1} e^{\gamma}}
$$


$2^{\circ}$ Upper bounds of $\Upsilon_{1}^{\prime}, \Upsilon_{2}^{\prime}$ and $\Upsilon_{3}^{\prime}$. We divide the interval $\left[x^{\kappa_{1}}, x^{\kappa_{2}}\right]$ into $O(\mathcal{L})$ subintervals of the form $[P, 2 P)$ and apply (2.4) of Lemma 2.2 to $S\left(\mathcal{A}_{p}^{\prime} ; \mathcal{P}(2), p\right)$ for $p \in[P, 2 P)$. We have

$$
\begin{aligned}
S\left(\mathcal{A}_{p}^{\prime} ; \mathcal{P}(2), p\right) \leq & \frac{\{1+O(\varepsilon)\} x}{\mathcal{L}} \frac{V(p)}{\varphi(p)} F\left(\frac{\log (Q / P)}{\log p}\right) \\
& +\sum_{l<L} \sum_{q \mid P(p)} \lambda_{l}^{+}(q) r\left(\mathcal{A}^{\prime}, p q\right)
\end{aligned}
$$

where $Q=x^{4 / 7-\varepsilon}$ and $\lambda_{l}^{+}(q)$ is well factorisable of level $Q / P$ and of order 1 .

Denote by $\pi_{P}$ the characteristic function of the primes in the interval $[P, 2 P)$. Since $P \leq x^{\kappa_{2}} \Rightarrow P \leq Q / P$, Lemma 2.1 shows that $\pi_{P} * \lambda_{l}^{+}$is well factorisable of level $Q$ and of order 2. Thus Lemma 2.7 allows us to deduce that

$$
\sum_{P \leq p<2 P} \sum_{l<L} \sum_{q \mid P(p)} \lambda_{l}^{+}(q) r\left(\mathcal{A}^{\prime}, p q\right) \ll_{\varepsilon} x /(\log x)^{4}
$$

and

$$
\Upsilon_{1}^{\prime} \leq \frac{\{1+O(\varepsilon)\} x}{\log x} \sum_{x^{\kappa_{1}} \leq p<x^{\kappa_{2}}} \frac{V(p)}{\varphi(p)} F\left(\frac{\log (Q / p)}{\log p}\right)+O\left(\frac{x}{(\log x)^{4}}\right) .
$$

Since $V(p) \sim e^{-\gamma} C / \log p$, the prime number theorem implies that

$$
\Upsilon_{1}^{\prime} \leq\left\{G_{1}+O(\varepsilon)\right\} \Pi(x)
$$

with

$$
G_{1}:=\frac{7}{4 e^{\gamma}} \int_{4 / 7 \kappa_{2}-1}^{4 / 7 \kappa_{1}-1} F(t) d t
$$

We divide the interval of summation $\left[x^{\kappa_{1}}, x^{\sigma_{1}}\right]$ of $\Upsilon_{2}^{\prime}$ into three parts:

$$
\left[x^{\kappa_{1}}, x^{2 / 7-\varepsilon}\right], \quad\left[x^{2 / 7-\varepsilon}, x^{29 / 100}\right], \quad\left[x^{29 / 100}, x^{\sigma_{1}}\right],
$$

and use (2.4) of Lemma 2.2 to handle each sum. As before we apply Lemma 2.7, the conditions (C.2) and (C.3) of Lemma 2.8, respectively, to control the corresponding error terms. We find

$$
\Upsilon_{2}^{\prime} \leq\left\{G_{2}+O(\varepsilon)\right\} \Pi(x),
$$

where

$$
\begin{aligned}
G_{2}:= & \frac{1}{\kappa_{1} e^{\gamma}}\left\{\int_{2 / 7 \kappa_{1}}^{4 / 7 \kappa_{1}-1} \frac{F(t)}{4 / 7 \kappa_{1}-t} d t+\int_{13 / 50 \kappa_{1}}^{2 / 7 \kappa_{1}} \frac{F(t)}{2 / \kappa_{1}-t} d t\right. \\
& \left.+\int_{\left(11 / 20-\sigma_{1}\right) / \kappa_{1}}^{13 / 50 \kappa_{1}} \frac{F(t)}{11 / 20 \kappa_{1}-t} d t\right\} .
\end{aligned}
$$


Similarly

$$
\Upsilon_{3}^{\prime} \leq\left\{G_{3}+O(\varepsilon)\right\} \Pi(x)
$$

where

$$
G_{3}:=\frac{1}{\kappa_{1} e^{\gamma}}\left\{\int_{2 / 7 \kappa_{1}}^{\left(4 / 7-\kappa_{1}\right) / \kappa_{1}} \frac{F(t)}{4 / 7 \kappa_{1}-t} d t+\int_{\left(2-6 \sigma_{2}\right) / \kappa_{1}}^{2 / 7 \kappa_{1}} \frac{F(t)}{2 / \kappa_{1}-t} d t\right\} .
$$

$3^{\circ}$ Lower bounds of $\Upsilon_{4}^{\prime}$ and $\Upsilon_{5}^{\prime}$. In view of $3 \kappa_{2} \leq 3 / 7<4 / 7$, a similar argument proving (11.2) implies that

$$
\Upsilon_{4}^{\prime} \geq\left\{G_{4}+O(\varepsilon)\right\} \Pi(x),
$$

where

$$
G_{4}:=\frac{1}{\kappa_{1} e^{\gamma}} \int_{\kappa_{1}}^{\kappa_{2}} \frac{d t}{t} \int_{t}^{\kappa_{2}} f\left(\frac{4 / 7-t-u}{\kappa_{1}}\right) \frac{d u}{u} .
$$

Our assumptions on $\kappa_{1}$ and $\varrho$ imply that $p_{1}^{2} p_{2} \leq x^{4 / 7-\varepsilon}$ and $p_{1}^{2} \leq x^{4 / 7-\varepsilon}$. As before we can apply (2.5) of Lemma 2.2 and Lemma 2.7 to get

$$
\Upsilon_{5}^{\prime} \geq\left\{G_{5}+O(\varepsilon)\right\} \Pi(x)
$$

where

$$
G_{5}:=\frac{1}{\kappa_{1} e^{\gamma}} \int_{\kappa_{1}}^{\kappa_{2}} \frac{d t}{t} \int_{\kappa_{2}}^{4 / 7-2 \kappa_{1}-t} f\left(\frac{4 / 7-t-u}{\kappa_{1}}\right) \frac{d u}{u} .
$$

$4^{\circ}$ Upper bounds of $\Upsilon_{i}^{\prime}$ for $i=7,8,9,10,13,14$. We shall apply the technique of [12]. Since $\sigma_{1}>2 / 7$, the quantity $\Upsilon_{7}^{\prime}$ is equal to the number of primes $p \leq x$ such that $p+2=p_{1} p_{2} p_{3}$ with $x^{\sigma_{1}} \leq p_{1}<p_{2}<\left(x / p_{1}\right)^{1 / 2}$ and $p_{3} \geq p_{2}$.

Introduce the set

$$
\mathcal{B}:=\left\{b-2: b=p_{1} p_{2} p_{3} \leq x, x^{\sigma_{1}} \leq p_{1}<p_{2}<p_{3}\right\} .
$$

Then we have

$$
\Upsilon_{7}^{\prime}=S\left(\mathcal{B} ; \mathcal{P}(2), x^{1 / 2}\right)+O\left(x^{1 / 2}\right) .
$$

Let $\Delta:=1+\mathcal{L}^{-4}$. We cover the set $\mathcal{B}$ by cuboids

$$
\mathcal{B}\left(t_{1}, t_{2}, t_{3}\right):=\left\{b-2: b=p_{1} p_{2} p_{3} \leq x, p_{i} \in\left[\Delta^{t_{i}}, \Delta^{t_{i}+1}\right) \text { for } 1 \leq i \leq 3\right\}
$$

where $t_{i}$ are integers satisfying $x^{\sigma_{1}} \leq \Delta^{t_{1}} \leq \Delta^{t_{2}} \leq \Delta^{t_{3}}$ and $\Delta^{t_{1}+t_{2}+t_{3}+3} \leq x$. In view of $x^{2 / 7} \leq p_{2} \leq x^{\left(1-\sigma_{2}\right) / 2} \leq x^{2 / 5}$, Lemma 2.5 with the choice

$$
\alpha_{m}=\left\{\begin{array}{ll}
1 & \text { if } m=p_{1} p_{3}, \\
0 & \text { otherwise }
\end{array} \quad \beta_{n}= \begin{cases}1 & \text { if } n=p_{2}, \\
0 & \text { otherwise }\end{cases}\right.
$$

implies the inequality

$$
\sum_{(q, 2)=1} \lambda_{l}^{+}(q)\left(\left|\mathcal{B}\left(t_{1}, t_{2}, t_{3}\right)_{q}\right|-\frac{\left|\mathcal{B}\left(t_{1}, t_{2}, t_{3}\right)\right|}{\varphi(q)}\right) \ll \frac{x}{(\log x)^{18}},
$$


where $\lambda_{l}^{+}(q)$ is well factorisable of order 1 and of level $Q=x^{\theta\left(t_{2}\right)}$ with $\theta\left(t_{2}\right)=\left(2+t_{2}\right) / 4$.

Thus we find by (2.4) of Lemma 2.2,

$$
S\left(\mathcal{B}\left(t_{1}, t_{2}, t_{3}\right) ; \mathcal{P}(2), x^{1 / 2}\right) \leq \frac{2 C\{1+O(\varepsilon)\}}{\theta\left(t_{2}\right) \mathcal{L}}\left|\mathcal{B}\left(t_{1}, t_{2}, t_{3}\right)\right|+O\left(\frac{x}{(\log x)^{18}}\right) .
$$

Since the number of cuboids $\mathcal{B}\left(t_{1}, t_{2}, t_{3}\right)$ is $\ll(\log x)^{15}$, we have

$$
\begin{aligned}
& \sum_{\left(t_{1}, t_{2}, t_{3}\right)} \frac{\left|\mathcal{B}\left(t_{1}, t_{2}, t_{3}\right)\right|}{\theta\left(t_{2}\right)} \\
& =\sum_{x^{\sigma_{1}} \leq p_{1}<p_{2} \leq\left(x / p_{1}\right)^{1 / 2}} \sum_{p_{1} p_{2} \log \left(x / p_{1} p_{2}\right)\left(2+\log p_{2} / \log x\right)}+O\left(\frac{x}{(\log x)^{2}}\right) \\
& =\frac{4 x\{1+O(\varepsilon)\}}{\log x} \iiint_{\sigma_{1} \leq t \leq u \leq(1-t) / 2} \frac{d t d u}{t u(1-t-u)(2+u)} .
\end{aligned}
$$

Combining these estimates, we obtain

$$
\Upsilon_{7}^{\prime} \leq\left\{G_{7}+O(\varepsilon)\right\} \Pi(x),
$$

where

$$
G_{7}:=8 \int_{\sigma_{1} \leq t \leq u \leq(1-t) / 2} \frac{d t d u}{t u(1-t-u)(2+u)} .
$$

Analogously we have

$$
\Upsilon_{8}^{\prime} \leq\left\{G_{8}+O(\varepsilon)\right\} \Pi(x)
$$

where

$$
G_{8}:=8 \int_{\sigma_{2} \leq t \leq u \leq(1-t) / 2} \frac{d t d u}{t u(1-t-u)(2+u)} .
$$

For $\Upsilon_{9}^{\prime}$, the assumption $3 \sigma_{1}+\kappa_{1}>1$ allows us to write

$$
\Upsilon_{9}^{\prime}=S\left(\mathcal{B}^{\prime} ; \mathcal{P}(2), x^{1 / 2}\right)+O\left(x^{1 / 2}\right)
$$

with

$$
\mathcal{B}^{\prime}:=\left\{b-2: b=p_{1} p_{2} p_{3} \leq x, x^{\kappa_{1}} \leq p_{1}<x^{\sigma_{1}} \leq p_{2}<p_{3}\right\} .
$$

We decompose

$$
\mathcal{B}^{\prime}=\mathcal{B}_{1}^{\prime} \cup \cdots \cup \mathcal{B}_{6}^{\prime},
$$

where $\mathcal{B}_{1}^{\prime}, \ldots, \mathcal{B}_{6}^{\prime}$ are defined as in (11.9) but we add the following extra conditions:

$$
\begin{aligned}
& p_{1} \leq x^{1 / 10} \text { in } \mathcal{B}_{1}^{\prime} ; \\
& p_{1}>x^{1 / 10} \text { and } p_{1} p_{2} \leq x^{1 / 2} \text { in } \mathcal{B}_{2}^{\prime} ; \\
& p_{1}>x^{1 / 10} \text { and } p_{1}^{-2} p_{2}^{8}>x^{3} \text { in } \mathcal{B}_{3}^{\prime} ;
\end{aligned}
$$




$$
\begin{aligned}
& p_{2}>x^{2 / 5} \text { and } p_{1}^{-2} p_{2}^{8} \leq x^{3} \text { in } \mathcal{B}_{4}^{\prime} ; \\
& x^{\left(1-\sigma_{2}\right) / 2}<p_{2} \leq x^{2 / 5} \text { and } p_{1} p_{2}>x^{1 / 2} \text { in } \mathcal{B}_{5}^{\prime} ; \\
& p_{2} \leq x^{\left(1-\sigma_{2}\right) / 2} \text { and } p_{1} p_{2}>x^{1 / 2} \text { in } \mathcal{B}_{6}^{\prime} .
\end{aligned}
$$

Again we can use Lemma 2.5 with

$$
\begin{array}{ll}
\theta\left(\log p_{1} / \log x\right)=\left(1+2 \log p_{1} / \log x\right) / 2 & \text { for } \mathcal{B}_{1}^{\prime} ; \\
\theta\left(\log p_{1} / \log x\right)=\left(5-2 \log p_{1} / \log x\right) / 8 & \text { for } \mathcal{B}_{2}^{\prime} \text { and } \mathcal{B}_{3}^{\prime} ; \\
\theta\left(\log p_{2} / \log x\right)=1-\log p_{2} / \log x & \text { for } \mathcal{B}_{4}^{\prime} ; \\
\theta\left(\log p_{2} / \log x\right)=\left(2+\log p_{2} / \log x\right) / 4 & \text { for } \mathcal{B}_{5}^{\prime} \text { and } \mathcal{B}_{6}^{\prime} .
\end{array}
$$

Then we have

$$
\Upsilon_{9}^{\prime} \leq\left\{G_{9}+O(\varepsilon)\right\} \Pi(x)
$$

where

$$
\begin{aligned}
& G_{9}=4 \underset{\substack{\kappa_{1} \leq t \leq \sigma_{1} \leq u \leq(1-t) / 2 \\
t \leq 1 / 10}}{\iint_{t u(1-t-u)(1+2 t)}} \\
& +16 \int_{\substack{\kappa_{1} \leq t \leq \sigma_{1} \leq u \leq(1-t) / 2 \\
t \geq 1 / 10, t+u \leq 1 / 2}} \frac{d t d u}{t u(1-t-u)(5-2 t)} \\
& +16 \int_{\substack{\kappa_{1} \leq t \leq \sigma_{1} \leq u \leq(1-t) / 2 \\
t \geq 1 / 10,8 u \geq 2 t+3}} \frac{d t d u}{t u(1-t-u)(5-2 t)} \\
& +2 \int_{\substack{\kappa_{1} \leq t \leq \sigma_{1} \leq u \leq(1-t) / 2 \\
u \geq 2 / 5,8 u \leq 2 t+3}} \frac{d t d u}{t u(1-t-u)(1-u)} \\
& +8 \int_{\substack{\kappa_{1} \leq t \leq \sigma_{1} \leq u \leq(1-t) / 2 \\
\left(1-\sigma_{1}\right) / 2 \leq u \leq 2 / 5, t+u \geq 1 / 2}} \frac{d t d u}{t u(1-t-u)(2+u)} \\
& +8 \underset{\substack{\kappa_{1} \leq t \leq \sigma_{1} \leq u \leq(1-t) / 2 \\
u \leq\left(1-\sigma_{1}\right) / 2, t+u \geq 1 / 2}}{\iint_{t u(1-t-u)(2+u)}} \underset{d t d u}{t u}
\end{aligned}
$$

Similarly in view of the assumption $2 \sigma_{1}+\sigma_{2}+\kappa_{2}>1$, we can prove that

$$
\Upsilon_{10}^{\prime} \leq\left\{G_{10}+O(\varepsilon)\right\} \Pi(x)
$$

where

$$
G_{10}=16 \int_{\substack{\kappa_{2} \leq t \leq \sigma_{2} \leq u \leq(1-t) / 2 \\ t+u \leq 1 / 2}} \frac{d t d u}{t u(1-t-u)(5-2 t)}
$$




$$
\begin{aligned}
& +16 \int_{\substack{\kappa_{2} \leq t \leq \sigma_{2} \leq u \leq(1-t) / 2 \\
8 u \geq 2 t+3}} \frac{d t d u}{t u(1-t-u)(5-2 t)} \\
& +2 \int_{\substack{\kappa_{2} \leq t \leq \sigma_{2} \leq u \leq(1-t) / 2 \\
2 / 5 \leq u \leq\left(2 \kappa_{2}+3\right) / 8}} \frac{d t d u}{t u(1-t-u)(1-u)} \\
& +2 \int_{\substack{\kappa_{2} \leq t \leq \sigma_{2} \leq u \leq(1-t) / 2 \\
u \geq\left(2 \kappa_{2}+3\right) / 8,8 u \leq 2 t+3}} \frac{d t d u}{t u(1-t-u)(1-u)}
\end{aligned}
$$

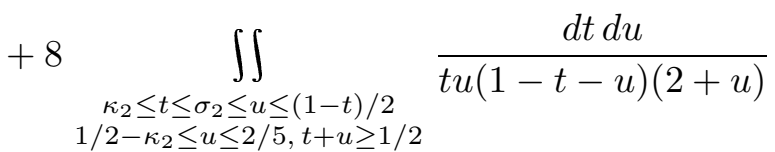

$$
\begin{aligned}
& +8 \quad \iint_{\substack{\kappa_{2} \leq t \leq \sigma_{2} \leq u \leq(1-t) / 2}} \frac{d t d u}{t u(1-t-u)(2+u)} \\
& \left(1-\sigma_{2}\right) / 2 \leq u \leq 1 / 2-\kappa_{2}, t+u \geq 1 / 2 \\
& +8 \underset{\substack{\kappa_{2} \leq t \leq \sigma_{2} \leq u \leq(1-t) / 2 \\
u \leq\left(1-\sigma_{2}\right) / 2, t+u \geq 1 / 2}}{\iint_{t u(1-t-u)(2+u)}} \underset{.}{t u d u}
\end{aligned}
$$

More easily we can prove that

$$
\Upsilon_{i} \leq\left\{G_{i}+O(\varepsilon)\right\} \Theta(N) \quad(i=13,14),
$$

where

$$
\begin{aligned}
G_{13}:= & 4 \int_{\kappa_{1}}^{1 / 10} \frac{d t_{1}}{t_{1}\left(1+2 t_{1}\right)} \int_{t_{1}}^{\kappa_{2}} \frac{d t_{2}}{t_{2}^{2}} \int_{t_{2}}^{\kappa_{2}} \frac{d t_{3}}{t_{3}} \\
& \times \int_{t_{3}}^{\kappa_{2}} \omega\left(\frac{1-t_{1}-t_{2}-t_{3}-t_{4}}{t_{2}}\right) \frac{d t_{4}}{t_{4}} \\
& +16 \int_{1 / 10}^{\kappa_{2}} \frac{d t_{1}}{t_{1}\left(5-2 t_{1}\right)} \int_{t_{1}}^{\kappa_{2}} \frac{d t_{2}}{t_{2}^{2}} \int_{t_{2}}^{\kappa_{2}} \frac{d t_{3}}{t_{3}} \\
& \times \int_{t_{3}}^{\kappa_{2}} \omega\left(\frac{1-t_{1}-t_{2}-t_{3}-t_{4}}{t_{2}}\right) \frac{d t_{4}}{t_{4}}
\end{aligned}
$$

and

$$
\begin{aligned}
G_{14}:= & 4 \int_{\kappa_{1}}^{1 / 10} \frac{d t_{1}}{t_{1}\left(1+2 t_{1}\right)} \int_{t_{1}}^{\kappa_{2}} \frac{d t_{2}}{t_{2}^{2}} \int_{t_{2}}^{\kappa_{2}} \frac{d t_{3}}{t_{3}} \\
& \times \int_{\kappa_{2}}^{4 / 7-2 \kappa_{1}-t_{3}} \omega\left(\frac{1-t_{1}-t_{2}-t_{3}-t_{4}}{t_{2}}\right) \frac{d t_{4}}{t_{4}}
\end{aligned}
$$




$$
\begin{aligned}
& +16 \int_{1 / 10}^{\kappa_{2}} \frac{d t_{1}}{t_{1}\left(5-2 t_{1}\right)} \int_{t_{1}}^{\kappa_{2}} \frac{d t_{2}}{t_{2}^{2}} \int_{t_{2}}^{\kappa_{2}} \frac{d t_{3}}{t_{3}} \\
& \times \int_{\kappa_{2}}^{4 / 7-2 \kappa_{1}-t_{3}} \omega\left(\frac{1-t_{1}-t_{2}-t_{3}-t_{4}}{t_{2}}\right) \frac{d t_{4}}{t_{4}} .
\end{aligned}
$$

Inserting these estimates and the trivial lower bounds $\Upsilon_{i}^{\prime} \geq 0(i=6,11,12,15)$ into (9.10), we obtain

$$
\pi_{1,2}(x) \geq\left\{G\left(\kappa_{1}, \kappa_{2}, \varrho, \sigma_{2}, \sigma_{1}\right)+O(\varepsilon)\right\} \Pi(x),
$$

where

$$
\begin{aligned}
G\left(\kappa_{1}, \kappa_{2}, \varrho, \sigma_{2}, \sigma_{1}\right):= & \frac{1}{4}\left(4 G_{0}-G_{1}-G_{2}-G_{3}+G_{4}+G_{5}\right. \\
& \left.-2 G_{7}-2 G_{8}-G_{9}-G_{10}-G_{13}-G_{14}\right) .
\end{aligned}
$$

If we set

$$
\kappa_{1}=\frac{2}{21}, \quad \kappa_{2}=\frac{13}{100}, \quad \varrho=\frac{2}{7}, \quad \sigma_{2}=\frac{36}{125}, \quad \sigma_{1}=\frac{332}{1000},
$$

a numerical computation gives

$$
\begin{aligned}
G\left(\kappa_{1}, \kappa_{2}, \varrho, \sigma_{2}, \sigma_{1}\right) \geq & \frac{1}{4}(4 \cdot 5.894705-1.611441-7.921437 \\
& -6.736885+0.270916+0.913995 \\
& -2 \cdot 0.000124-2 \cdot 0.145114 \\
& -1.790090-1.930545-0.006814-0.059690) \\
> & 1.10409 .
\end{aligned}
$$

[For the integrals $F_{13}$ and $F_{14}$, we make use of $\omega(u) \leq 0.561522$ for $u \geq 3.5$ and $\omega(u) \leq 0.567144$ for $u \geq 2$.] This proves Theorem 4 .

\section{References}

[1] E. Bombieri and H. Davenport, Small differences between prime numbers, Proc. Roy. Soc. Ser. A 293 (1966), 1-18.

[2] E. Bombieri, J. B. Friedlander and H. Iwaniec, Primes in arithmetic progressions to large moduli, Acta Math. 156 (1986), 203-251.

[3] Y. C. Cai, A remark on Chen's theorem, Acta Arith. 102 (2002), 339-352.

[4] Y. C. Cai and M. G. Lu, Chen's theorem in short intervals, ibid. 91 (1999), 311-323.

[5] - - - On Chen's theorem, in: Analytic Number Theory (Beijing/Kyoto, 1999), Dev. Math. 6, Kluwer, Dordrecht, 2002, 99-119.

[6] - - - On the upper bound for $\pi_{2}(x)$, Acta Arith. 110 (2003), 275-298.

[7] J. R. Chen, On the representation of a large even integer as the sum of a prime and the product of at most two primes, Sci. Sinica 16 (1973), 157-176.

[8] - On the representation of a large even integer as the sum of a prime and the product of at most two primes (II), ibid. 21 (1978), 421-430. 
[9] J. R. Chen, Further improvement on the constant in the proposition '1+2': On the representation of a large even integer as the sum of a prime and the product of at most two primes (II), ibid. 21 (1978), 477-494 (in Chinese).

[10] - On the Goldbach's problem and the sieve methods, ibid. 21 (1978), 701-739.

[11] E. Fouvry, Autour du théorème de Bombieri-Vinogradov, Ann. Sci. École Norm. Sup. 20 (1987), 617-640.

[12] E. Fouvry and F. Grupp, On the switching principle in sieve theory, J. Reine Angew. Math. 370 (1986), 101-125.

[13] - - - Weighted sieves and twin prime type equations, Duke Math. J. 58 (1989), 731-748.

[14] H. Halberstam and H.-E. Richert, Sieve Methods, Academic Press, London, 1974.

[15] G. H. Hardy and J. E. Littlewood, Some problems of 'partitio numerorum' III: On the expression of a number as a sum of primes, Acta Math. 44 (1923), 1-70.

[16] H. Iwaniec, Primes of the type $\phi(x, y)+A$ where $\phi$ is a quadratic form, Acta Arith. 21 (1972), 203-234.

[17] -, A new form of the error term in the linear sieve, ibid. 37 (1980), 307-320.

[18] H. Iwaniec and J. Pomykała, Sums and differences of quartic norms, Mathematika 40 (1993), 233-245.

[19] C. B. Pan, On the upper bound of the number of ways to represent an even integer as a sum of two primes, Sci. Sinica 23 (1980), 1367-1377.

[20] C. D. Pan, A new application of the Yu.V. Linnik large sieve method, Chinese Math. Acta 5 (1964), 642-652.

[21] C. G. Pan and X. X. Ding, A new mean value theorem, Sci. Sinica (1979), Special Issue II on Math., 149-161.

[22] C. D. Pan and C. B. Pan, Goldbach Conjecture, Science Press, Beijing, 1992.

[23] P. M. Ross, A short intervals result in additive prime number theory, J. London Math. Soc. (2) 17 (1978), 219-227.

[24] S. Salerno and A. Vitolo, $p+2=P_{2}$ in short intervals, Note Mat. 13 (1993), 309-328.

[25] A. Selberg, On elementary methods in primenumber-theory and their limitations, in: Den 11te Skandinaviske Matematikerkongress (Trondheim, 1949), Johan Grundt Tanums Forlag, Oslo, 1952, 13-22.

[26] J. Wu, Sur la suite des nombres premiers jumeaux, Acta Arith. 55 (1990), 365-394.

[27] —, Théorèmes généralisés de Bombieri-Vinogradov dans les petits intervalles, Quart. J. Math. Oxford (2) 44 (1993), 109-128.

[28] - Sur l'équation $p+2=P_{2}$ dans les petits intervalles, J. London Math. Soc. (2) 49 (1994), 61-72.

Institut Elie Cartan

UMR 7502 UHP-CNRS-INRIA

Université Henri Poincaré (Nancy 1)

54506 Vandœuvre-lès-Nancy, France

E-mail: wujie@iecn.u-nancy.fr

Received on 14.5.2003

and in revised form on 18.9.2003 